\title{
CORROSION AND ARC EROSION IN MHD CHANNELS
}

Final Report

By

Richard J. Rosa

Richard J. Pollina

August 1992

Work Performed Under Contract No. FG22-88PC88928

For

U.S. Department of Energy

Plttsburgh Energy Technology Center

Pittsburgh, Pennsylvania

By

Montana State University

Bozeman, Montana 


\title{
DISCLAIMER
}

\begin{abstract}
This report was prepared as an account of work sponsored by an agency of the United States Government. Neither the United States Government nor any agency thereof, nor any of their employees, makes any warranty, express or implied, or assumes any legal liability or responsibility for the accuracy, completeness, or usefulness of any information, apparatus, product, or process disclosed, or represents that its use would not infringe privately owned rights. Reference herein to any specific commercial product, process, or service by trade name, trademark, manufacturer, or othervise does not necessarily constitute or imply its endorsement, recommendation, or favoring by the United States Government or any agency thereof. The views and opinions of authors expressed herein do not necessarily state or reflect those of the United States Government or any agency thereof.
\end{abstract}

This report has been reproduced directly from the best available copy.

Available to DOE and DOE contractors from the Office of Scientific and Technical Information, P.O. Box 62, Oak Ridge, TN 37831; prices available from (615)576-8401.

Available to the public from the National Technical Information Service, U.S. Department of Commerce, 5285 Port Royal Rd., Springfield, VA 22161. 
FINAL REPORT

DE-FG22-88PC88928

\section{CORROSION AND ARC EROSION IN MHD CHANNELS}

CO-PRINCIPAL INVESTIGATORS

RICHARD J. ROSA

RICHARD J. POLLINA*

\section{DEPARTMENT OF MECHANICAL ENGINEERING MONTANA STATE UNIVERSITY \\ BOZEMAN, MT 59717}

AUGUST, 1992

* also at E.G. and G/EM R56-14, P.O. Box 1912, Las Vegas, NV 89125 
Page

Title Page

Table of Contents

List of Figures

Executive Summary

1. Introduction 1

2. Corrosion 2

2.1 Introduction 2

2.2 Gas Side Corrosion Wear Data and Lifetime Estimates 2

2.3 Gas Side Corrosion Analysis 11

2.4 Water Corrosion 17

2.4.1 Water Corrosion Test Results 17

$\begin{array}{ll}2.5 \text { Conclusions } & 19\end{array}$

2.6 References 21

3. Current Distribution; Introduction 23

3.1 The Faraday Voltage Profile Method 24

3.2 The Load Sweep Method 43

3.3 The Modeling Method 49

3.4 Conclusions 52

3.5 Recommendations $\quad 58$

3.6 References $\quad 59$

4. Acknowledgement 60

Appendix A 61

Final Report Distribution List $\quad 62$ 
Section 2.

List of Figures

Fiqure

Page

1. Four Possible Anode Designs with the Intended Channel Locations Indicated.

2. Conceptual Schematic of Plasma Current and Boundary Layer Ions in the Channel.

3. Observed Distribution of Anode Arcs in Coal-Fired 1Al Tests.

4. Calculation of Estimated Life of a Pt-Clad Tungsten Anode.

5. Method of Calculating the Life of a Tungsten Anode.

6. Candidate Cathode and Sidewall Element Design. The dashed line indicates the possibility of a brazed refractory cap on a refractory base.

\section{Section 3.}

\section{Figure}

Page

1.1 Comparison of inferred conductivity under load with measured conductivity while unloaded.

1.2 Typical plot of apparent leakage current vs axial field obtained during a load sweep. Condition numbers correspond to those in the Preliminary Data Report issued by MSE.

1.3 Apparent leak current vs measured Faraday (transverse) current density for runs prior to and including 89-DIAG-4, al1 for approximately the same axial electric field.

1.4 Apparent leak current vs measured Faraday current density for runs 89-nIAG-4 through 90-DIAG-13, and a range of axial fields.

1.5 Leak current vs Faraday current, jy, at several axial locations.

1.6 Leak current vs $\left(\beta^{*} j y\right)$ at several axial locations.

1.7 Leak current vs the square of $\left(B^{\star} j y\right)$ at several axial locations.

1.8 Apparent leak current vs electrode current for iron on, iron off, ash injected oil combustion (AIC), and a no iron-no polarization condition. Data from the Mark 6, Mark 7, and CDIF electrode locations $71,99,126$, and 139 .

1.9. Same as figure 1.8 but with data from CDIF electrode locations 126 and 139 only. 
1.10 Finite segmenting effect magnified by shorted cathode gaps. 38

1.11 Finite segmenting effect magnified by plasma streamers. $\quad 40$

2.1 Load current density vs Hall field for electrodes 90 through 130.44

2.2 Faraday current density vs Hall field for electrodes 90 through $130 \quad 45$

2.3 Sweep diagnosis of run 90-DIAG-3 for resistivity, rsc, Hall 47 parameter, $\beta$, and nonuniformity factor, $G$.

2.4 Sweep diagnosis of run 90-DIAG-3 for surface voltage drop, Ves1, 48 and slag conductivity, $\sigma$ slag.

2.5 Least squares fit to the data in figure 2.4. 48

2.6 Hall parameter vs axial position. $\quad 48$

3.1 Hall field vs axial position for a load current of 231 Amp. 50

3.2 Faraday current density vs axial position for a load current of 50 231 Amp.

3.3 Hall field distribution for a load current of 414 Amp. 51

3.4 Faraday current distribution for a load current of 414 Amp. 51

3.5 Unloaded electrical conductivity distribution. 51

4.1 Net leakage current vs hall field for $\beta=2, u B=4200 \mathrm{v} / \mathrm{m}$, channel 56 dimensions $Y$ and $Z$ are 0.3 and $0.2 \mathrm{~m}$ respectively, diagonal connection angle $=11 * .0155 / Y$, transverse resistivity, $\langle 1 / \sigma\rangle=0.2$.

4.2 Net leakage current vs Faraday current for indicated Hall fields. other parameters as in figure 3.6. 


\section{EXECUTIVE SUMMARY}

The objective of this work has been to study problems critical to the reliability of an MHD generator, specifically the corrosion, electrical heating, and arc erosion of MHD electrodes and side-wall elements. It has been a cooperative effort in support of the Department of Energy's Coal-fired MHD Topping Cycle Program and the Proof of Concept (POC) test series. Data on materials and electrical behavior have come from the Avco/Textron MHD test facility and the D.O.E. Component Development and Integration Facility (CDIF).

The problems connected with gas side corrosion for the design of the IA4 (POC) channel hardware are explored and results of gas side wear rate tests in the Textron Mark VII facility are presented. It is shown that the proposed designs meet a 2000 hour lifetime criterion based upon these materials tests. Improvement in cathode lifetime is demonstrated with lower voltage intercathode gaps. The corrosion of these materials is discussed and it is shown how iffetimes are dependent upon gap voltage and average metal temperature. The importance of uniformity of slagging to the durability of the anode wall is demonstrated. The wear mechanism of the anodes in the MHD channel is analyzed.

In addition to gas-side corrosion, the results of specific water corrosion tests of sidewall materials are discussed. All of the tests reported here were carried out to confirm the gas-side performance and the manufacturability of anode and sidewall designs and to address questions posed about the durability of tungsten-copper on the waterside. the results of water corrosion tests of the tungsten copper alloy sidewall material are presented to show that with proper control of waterside $\mathrm{pH}$ and, if necessary, dissolved oxygen, one can obtain reliable performance with no degradation of heat transfer with this material. The final choice of materials was determined primarily by the outcome of these tests and also by the question of the manufacturability of the prospective designs.

Questions regarding the distribution of electric current within the MHD channel are explored because the distribution affects local gas-side surface temperature. This temperature in turn strongly affects corrosion rate. The measured gas-side rates in such places as the upstream edge of anodes and the downstream edge of cathodes imply that local elevation of surface temperature by the electric current must play an important role. The local distribution of current is not measured directly, and it is difficult to do so, but some of it's features can be inferred from the electrical performance data that is routinely measured during generator testing at the CDIF and Avco/ Textron. In particular, axial leakage current can be estimated. This current is presumed to flow primarily on the cathode wall. It may flow entirely within the slag layer, or it may flow directly between cathodes causing localized heating and subsequent accelerated corrosion.

Data gathered from many CDIF runs suggests that axial current leakage is quite high, especially in the upstream half of the duct. Moreover, this apparent leakage turns out to be proportional not to axial Hall voltage but to Faraday current. A study of mechanisms that might lead to this unexpected dependence was carried out. It is concluded that the shorting of cathodes together, in groups of about two or more is one of the more likely mechanisms.

This group shorting, called "resegmentation", is commonly observed in generators fired with western coal having a low iron content. When an iron slurry is injected at the entrance to the channel the direct evidence of shorting disappears. In other words, all interelectrode gaps support a voltage. However, 
both the power output and the apparent leakage are observed to change only slightly. This could be due to a high rate of deposition of iron oxide slurry and/or seed on the upstream walls making the top surface of the slag layer so highly conducting as to lead to a virtual shorting and resegmentation process there, which would not be apparent from interelectrode voltmeter readings. Leakage along the top surface may not directly cause excessive heating of wall elements. However it may do so indirectly by causing voltage nonuniformities. The effect on power output is expected to scale like channel perimeter and thus diminish in importance as generator size increases. 


\section{INTRODUCTION}

The objective of this work has been to study problems critical to the reliability of an MHD generator, specifically the corrosion, electrical heating, and arc erosion of MHD electrodes and side-wall elements. It has been a cooperative effort in support of the Department of Energy's Coal-fired MHD Topping Cycle Program and the Proof of Concept (POC) test series. Data on materials and electrical behavior have come from the Avco/Textron MHD test facility and the D.O.E. Component Development and Integration Facility (CDIF). Because corrosion in an MHD generator is strongly influenced by the electrical current distribution the work has consisted of two tasks; corrosion, and current distribution. The former is described in Section 2 and the latter in Section 3. 


\section{CORROSION}

\subsection{INTRODUCTION}

Critical to the reliability of an MHD generator is the choice of materials of construction. The most critical and the most visible issue is that of the gas side wear of channel electrodes and sidewalls. No less important, especially from a plant operator's point of view, is the question of resistance to water side corrosion. The choice of materials for the IA4 channel to be used in the Proof of Concept(POC) test series involves a complex interplay of gas-side erosion and corrosion issues, water side corrosion, the MHD performance of the prospective materials, and materials integration issues. Materials integration issues include the questions of thermal and mechanical compatibility of the materials(gas side caps brazed to water side bases into which one brazes water tubes, plugs, and electrical studs), reliable manufacturability with satisfactory process yields, the relative ease and thoroughness of quality control inspection procedures of the final brazed assemblies, and the more elusive questions of latent failure potentialities and ability to hold up under assembly and disassembly stresses in the field.

This report addresses both the gas side corrosion issue and the results of water side corrosion. It summarizes results of work done at the AVCO Research Laboratory/Textron and which were reported on earlier. ${ }^{1},{ }^{2},{ }^{3}, 4$ The goal of the corrosion work was not to determine the single best material for a channel wall but to provide a selection of choices, each with varying degrees of corrosion resistance, which could then be folded into the other issues listed above and from which suitable final candidates were chosen. The issues are far too complex for decisions to be quick and easy.

\subsection{GAS SIDE CORROSION WEAR DATA AND LIFETIME ESTIMATES}

For ali practical purposes gas side wear can be reduced to two general categories, anode wear and cathode wear. Sidewall wear can be a subset of either but observations have shown that most sidewall wear is similar to that of the cathode, by which we mean that it wears via similar mechanisms. Very little wear is observed In the Mark VII tests on the sidewall elements adjoining the anode wa11. In fact aluminum end pegs performed remarkably well in Mark VII power tests and showed little or no wear after several hundred hours. Their projected lifetime would easily have surpassed 2000 hours. Extensive testing was therefore concentrated on anode and cathode designs to determine wear resistance of materials on those walls, but only back-up verification testing was done on the sidewalls, since sidewall performance so well parallels that of the cathode.

\section{ANODES:}

The four prototypical anode desigris which had been considered for the IA4 are shown in Figure 1 . The distinctive feature of each is the $1 / 8 \times 1 / 8$ " platinum corner on the upstream side of the anode. That the platinum leading corner bears the brunt of the current carrying activity of the anode is shown schematically in Figure 2 in which the current streami ines emanate from this point of the anode and are collected by the cathode trailing edge. This corner bears $90 \%$ of the arc activity as shown by the CDIF data presented in Figure 3. 

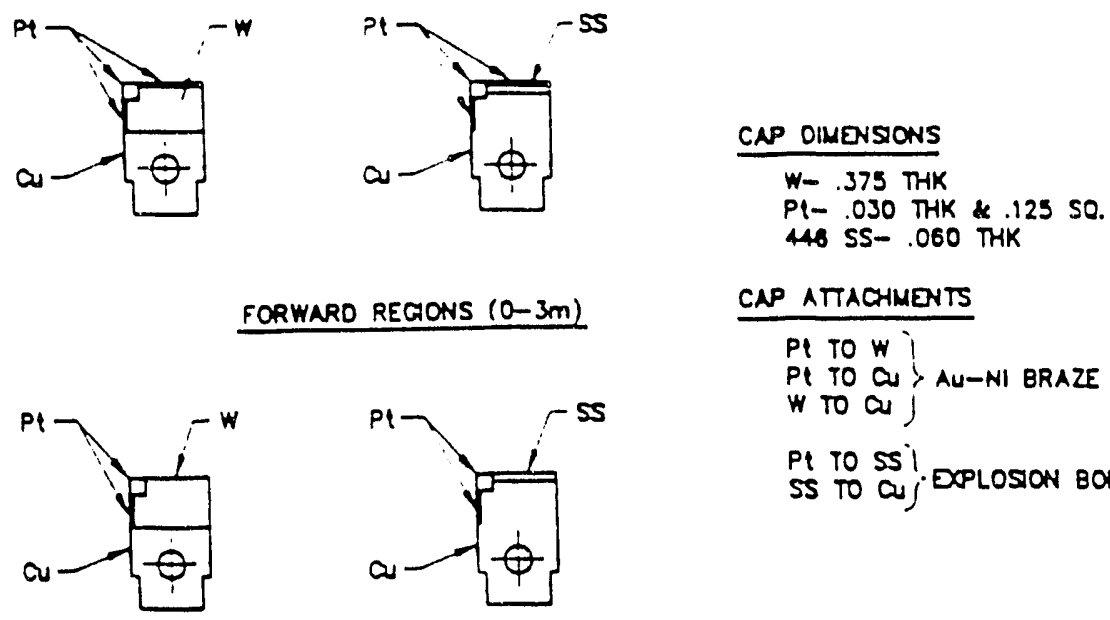

FORWARD REGONS $(0-3 \mathrm{~m})$

CAP ATTACHMENTS

Pt TO $W$

Pi TO QU C AU-NI BRAZE

W To a)

$\begin{array}{lll}\text { PI TO } & \text { SS' } \\ \text { SS TO } & \text { CUj DPLOSON BONO }\end{array}$

REAR REGONS (3-4m)

Figure 1. Four Possible Anode Designs with the

Intended Channel Locations Indicated

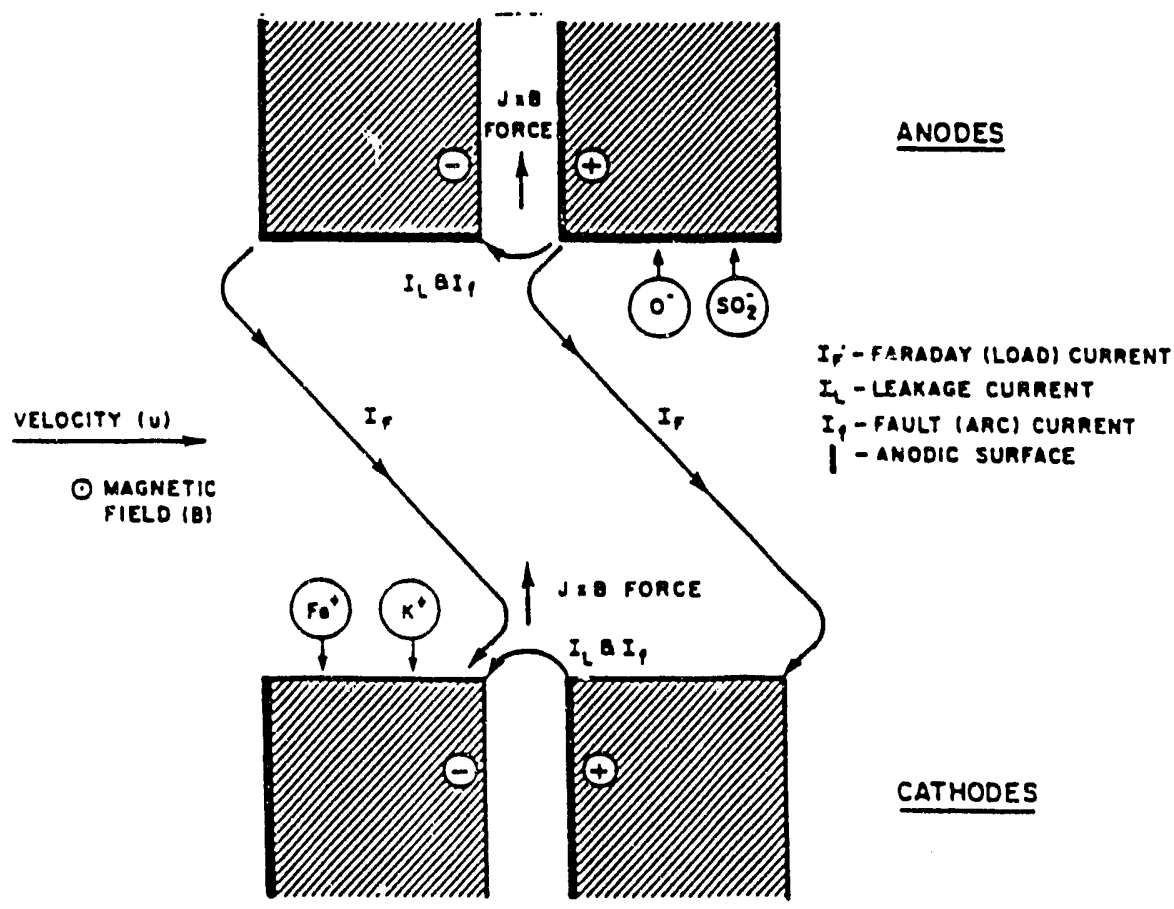

Figure 2. Conceptual Schematic of Plasma Current and Boundary Layer Ions in the Channel 
Other points illustrated in Figure 2 are the interanode arcs which are driven into the wall by the $J \times B$ force and the intercathode leakage current which produces most of the cathode wall wear on the anodic or upstrean facing edge of the cathodes. For both walls we indicate the chemical species peculiar to each wall but do not show the ubiquitous presence of $20 \% \mathrm{H}_{2} \mathrm{O}$ in the gas stream, which is likely to be a major source of corrosion for the refractory metals used. We also do not show the potential for sidewall-electrode arcs which primarily wear the leading edge of the cathodes, especially in the presence of high cathode wall nonuniformities.

The arc erosion caused by anode faults occurs on the upstream side of the anode below the gas surface. Arcs which initiate between anodes at the gas side are driven by the magnetic field between the anodes in the direction of the backwall. Characteristic damage due to these arcs is an erosion track on the upstream anode face extending from just below the platinum corner to the point of its termination. If this point of termination is the wall, charring can occur which can carbonize the wall sufficiently to cause interanode shorts to develop. This is a primary concern in the operation of the channel. The anode wear caused by these arcs below the gas surface is not a factor in anode life for the following reasons:

1. Platinum foil is brazed to the leading edge of the anode just below the corner to eliminate wear of the copper base in this region.

2. The use of current controls stabilizes the inter anode voltages and thereby reduces the risk of an anode fault.

3. The high thermal diffusivity and the low operating temperature of the copper base in contact with $B N$ insulation serves as an unfavorable thermal environment in which to sustain an arc.

4. An arc suppression groove(the reduced width visible at the base of each anode shown in Figure 1) is cut on each side of the anode near its base. This stretches the arc path as the arc is driven by the magnetic field towards the wall and this stretching serves to quench the arc.

Table 1 shows the available wear data for Pt anodes. Rates have been normalized to $1 \mathrm{~A} / \mathrm{cm}^{2}$ current density and a plasma sulfur percentage of $0.18 \%$, the level of $S$ obtained using Rosebud coal. Note that recession rates are given in two units: weight loss per coulomb and in measured cross sectional area of the Pt corner lost $\left(\mathrm{mil}^{2}\right)$. All but the last entry is total electrode weight loss per coulomb. Since total weight was measured in the first three entries, this data includes the wear of the entire piece tested and so is the sum of corner, top and Pt leading edge wear. The next four entries are results from the British MHD program and are also based upun Pt plus Cu weight loss, but the erosion losses of Pt should be higher for this data since the exposure of the Pt was to a particle stream impinging normal to the surface. The exposure times may also have been too short to accumulate appreciable water-side erosion of the $\mathrm{Cu}$. The final entry is the result of 152 hours of testing of a Pt clad, stainless steel capped anode in the Mark VII generator at a sulfur level of $0.27 \%$ in the plasma. This anode was similar to that shown in the upper right-hand side of Figure 1. 
TABLE 1

RECESSION RATES* OF PLATINUM ANODES IN MHD TESTS

Origin of data is indicated.

\section{DURATION}

$1000 \mathrm{hr}$

50

92

$-\cdot$

$-$

$-\cdot$

22

152
RECESSION RATES

$0.11 \mu \mathrm{G} / \mathrm{C}$

0.21

0.15

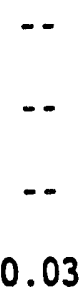

$4.4 \mathrm{MIL}^{2} / H R^{\star *}$

$8.4 * *$

6.0

3.2

1.9

$6.4(0.7)^{\star \star \star \star}$

1.2

3.6

\section{REFERENCE}

AVCO REPORT, 1000 HR TEST

V.J. HRUBY, ET AL., SEAM 19

V.J. HRUBY, $\mathrm{K}_{2} \mathrm{SO}_{4}$ TEST \#6

HEYWOOD-WOMACK TABLE 7.6

1

FIGURE 7.8

TABLE 7.5

MARK VII-CURRENT TESTS

* RATES ARE NORMALIZED TO $J=1 A / \mathrm{CM}^{2}$. PLASMA $S=0.18 \%$

** 6.7 MIL ${ }^{2} /$ HR TRANSLATES INTO PT LIFE OF 2000 HRS ASSUMING $90 \%$ OF $\mathrm{J}$ AT CORNER

*** RECESSION RATE AT $S=0.45 \%$ IS MEASURED TO BE $0.31 \mu \mathrm{G} / \mathrm{C} . \quad 12.4 \mathrm{CM}^{2} / \mathrm{HR}$

$\star * \star *$ VALUE USED IS HIGHEST OF SEVERAL TECHNIQUES. THIS VALUE FOR $\mathrm{K}_{2} \mathrm{SO}_{4} \mathrm{SEED}$. NOT NORMALIZED TO $0.18 \% \mathrm{~S}$. VALUE IN PARENTHESIS IS FOR $\mathrm{K}_{2} \mathrm{CO}_{3}$ SEED.

Initial anode wear occurs at angles of 30 to 60 degrees in the Pt corner, so for the sake of calculations we shall assume a 45 degree angle to the surface for the $\mathrm{Pt}$ corner wear. There are two transition points in wear rate calculations. The first occurs when the corner is worn past the foil cap and edge, exposing $W$ and ss in their respective designs. This region has wear rates characteristic of Pt wear. On crossing this first transition point the wear rate then becomes that due to $W$ and ss respectively and the wear angle has been experimentally seen to be at 30 degrees(shallow) to the surface. The second transition point comes when the $W$ or ss is worn to the point that the $\mathrm{Cu}$ is breached. This point is regarded as the life expectancy point. Both transition points are shown in Figure 5 for the Pt clad W design. For Pt-clad anodes the first transition point is expected to come at 3000 hours based upon the data in Table 1. This line is shown as the cut through the Pt corner in Figure 4.

In the case of the rear anodes with only the Pt corner and no foil on top, the 50 percent corner life point is 4000 hours because they operate at a current density, $0.5 \mathrm{~A} / \mathrm{cm}^{2}$, which is greatly reduced in comparison to the forward anodes, for which $1 \mathrm{~A} / \mathrm{cm}^{2}$ was used. This is twice the 2000 hours it takes to exactiy bisect the $\mathrm{Pt}$ corner at $1 \mathrm{~A} / \mathrm{cm}^{2}$.

In order to calculate the remaining life of the anodes, assuming the Pt 


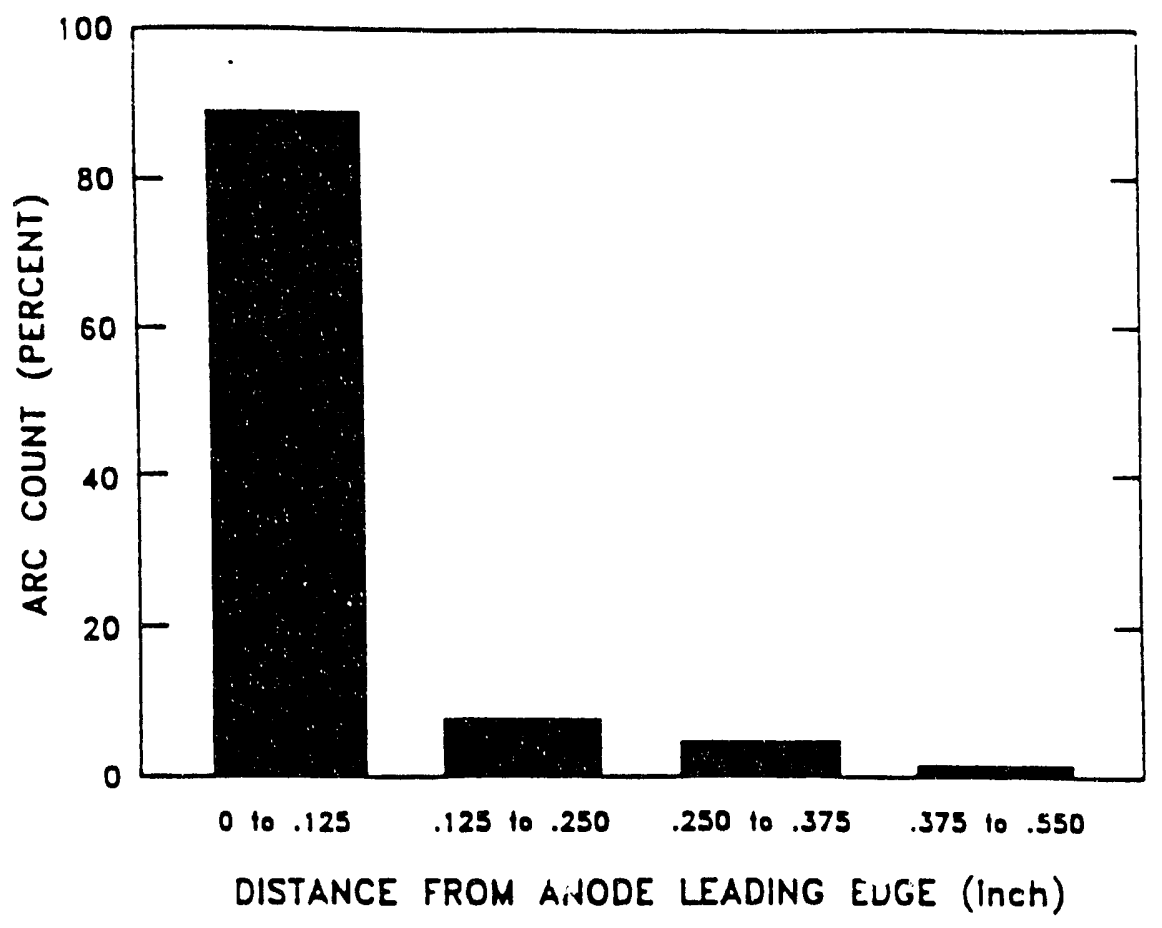

Figure 3. Observed Distribution of Anode Arcs in Coal-Fired IAl Tests

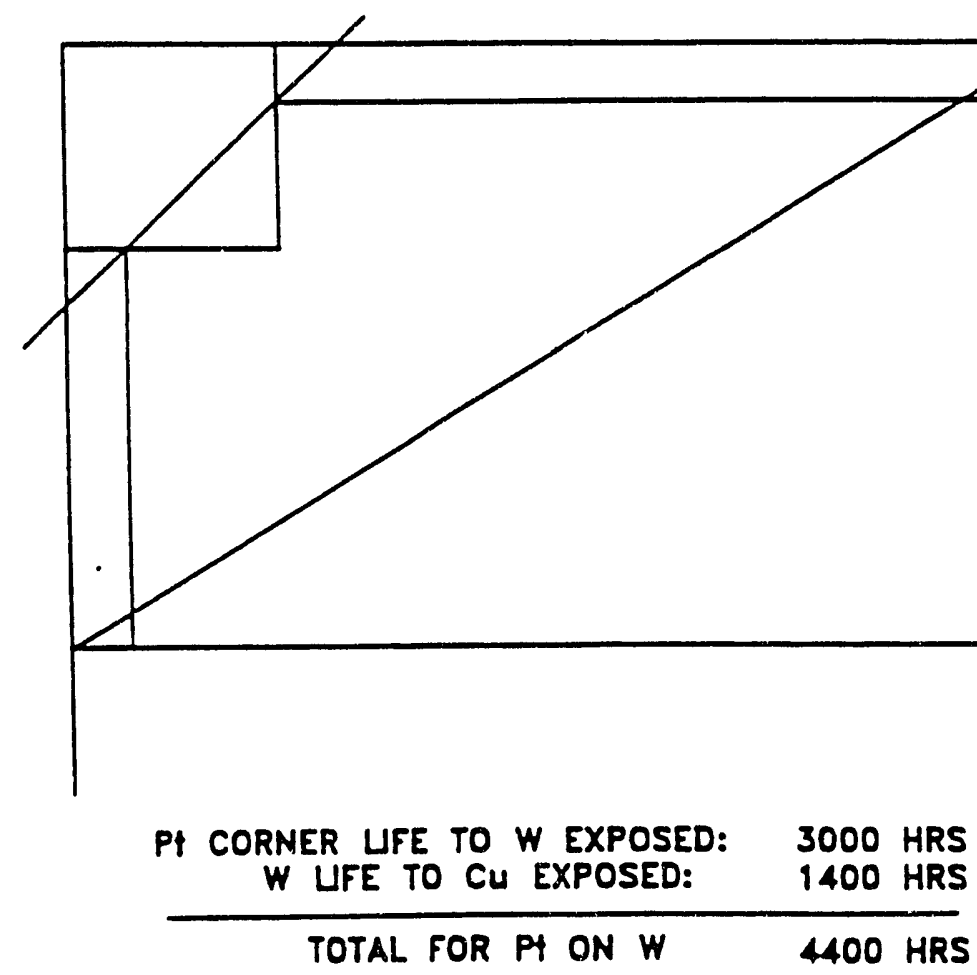

Figure 4. Calculation of Estimated Life of a Pt-Clad Tungsten Anode 
corner plays no role after the 50 percent wear point, we need the recession rate data for $W$ and Ss without a Pt corner. Data for the recession rates of anode back-up materials is shown in Table 2. This is the time necessary to reach the second transition line, which breaches the $\mathrm{Cu}$, shown in Figure 4.

Figure 5 shows how the calculation was done using the data in Table 2 for the lifetime of a $W$ anode without a Pt corner. Testing wore the hatched area shown in the corner, it is a simple matter to estimate the time for passage into the $\mathrm{Cu}$ with 30 degree angle wear. The result is that a $W$-capped anode should last 1400 hours. This does not take into account the remaining $P t$ left in the corner at the first rate transition point.

TABLE 2

RECESSION RATES* OF ANODE BACK-UP MATERIALS

IN MHD POWER TESTS

\begin{tabular}{|c|c|c|c|c|}
\hline DURATION & MATERIAL & RECESSIO & ON RATE** & REFERENCE \\
\hline $1000 \mathrm{HRS}$ & SS W.PT CORNER & $0.24 \mu \mathrm{G} / \mathrm{C}$ & & AVCO REPORT, 1000 HR TEST \\
\hline 50 HRS & 446SS (NO PT CORNER) & 0.67 & $73 \mathrm{MIL}^{2} / H R^{\star \star \star}$ & V.J. HRUBY, ET AL, SEAM 19 \\
\hline 50 HRS & W TOP CAP (ONLY) & & 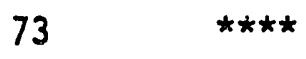 & MARK VII \\
\hline $50 \mathrm{HRS}$ & CU & 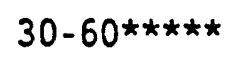 & $5000 t$ & V.J. HRUBY, ET AL. \\
\hline
\end{tabular}

* RATES ARE NORMALIZED TO $\mathrm{J}=1 \mathrm{~A} / \mathrm{CM}^{2}$. PLASMA $S=0.18 \%$

** $2000 \mathrm{HR}$ LIFE IS $59 \mathrm{MIL}^{2} /$ HR FOR W(1OMM CAP) AND $1.6 \mathrm{MIL}^{2} /$ HR FOR SS(1.5MM)

$\star \star \star \quad$ RECESSION RATE MEASURED AT $S=0.45 \%$ IS $1.7 \mu \mathrm{G} / \mathrm{C}$

$\star \star \star \star$ THIS IS AVERAGE VALUE OF WORST WEAR POINTS.

$\star \star \star \star \star$ RECESSION RATE AT $S=0.45 \%$ IS $140 \mu \mathrm{g} / \mathrm{C}$

For ss the situation is not so simple since the remaining $P t$ is a significant factor in the life of the anode between the first and second transition points. However once the ss has been breached, most often just behind the Pt corner or at the corner itself if there is no Pt protection, it will begin to attract more and more of the anode current density away from the corner because of its higher temperature. This results in an additional life which is difficult to estimate. Wear rates indicate that $60 \mathrm{mil}$ thick ss without a Pt corner will last 50 hours. But with some Pt present it could be much longer. Thus for the life expectancy of the primary designs we obtain 4400 hours for the Pt clad W design, noted in Figure 4, and in excess of 3050 hours for the Pt clad ss design. These are tabulated in Table 3 along with the results for the rear anode designs. The rear anode life estimates utilize the 4000 hour low current density life of the Pt corner as well as the increased lifetimes, 2800 and 100 hours, of $W$ and ss respectively. The results for the anode lifetime estimates clearly show the superiority of the $W$-capped anode over the ss-capped design. 
TABLE 3

LIFE EXPECTANCY OF MHD ANODE DESIGNS

\section{DESIGN}

Forward Pt over $W$

Forward Pt over ss

Rear W with Pt $\left(.5 \mathrm{a} / \mathrm{cm}^{2}\right)$

Rear ss with Pt $\left(.5 \mathrm{a} / \mathrm{cm}^{2}\right)$
LIFE

$4400 \mathrm{Hr}$

$>3050$

6800

4100

\section{CATHODES AND SIDEWALL:}

From near the sidewall midline to the anode wall the sidewall wear may be as little as one tenth that of the cathode wall and so does not concern us here. In the IAl the bar sidewall has a single long cathode bar extending to the midlint. This is not enough segmentation of the sidewall and leads to wear at the midline. In the modified Z-bar sidewall design for the $1 A 4$, there are three elements on the sidewall along a bar diagonal from the cathode to the midline. This segmentation of the sidewall reduces the midline voltage stresses thereby reducing wear there. The wear of the sidewall elements closest to the cathode may be approximated to that of the cathode.

Figure 6 shows an outline of the basic cathode and sidebar configurations considered for the 1A4. As shown, one candidate design for the sidewall was an all refractory design which allowed for the possibility of a separately brazed refractory cap on a refractory base(broken line). Table 4 gives the wear rate data for selected cathode materials as of April, 1990. All of the data was obtained on the Mark VII generator with the exception of the last 75W25Cu(sintered 75W-25Cu) entry. This is CDIF data obtained with the TRW coal combustor and is reported on el sewhere. ${ }^{5}$ With the exception of the 75W25Cu data shown between the two broken lines, all of the data was taken at high voltage intercathode gaps(100-160 volts) characteristic of the 1Al before the use of iron oxide. That is, the wear data at high gaps was taken from a series of high channel leakage current tests which were performed with all but 8 gaps of a 65 electrode channel shorted. These wear rates are considerably higher than those obtained under normal conditions and are indicated in the table. We have included them for completeness and to underscore the fact that operating channels off design can lead to much higher wear rates than anticipated. This gives higher wear rates than one would expect with the use of iron oxide in the IAl where its use drives the inter cathode voltages down to 20-25 volts. In all but the 450 hour data the values shown were measured at the worst wear point. The 450 hour data was obtained from a distribution of average wear rates at the indicated average voltage(20 and 50 volts). Worst wear means that the deepest wear point was recorded. No averaging was done over the length of an electrode. For groups of similar electrodes worst wear data for each electrode was averaged over the group. By doing this we attempted to be as conservative as possible in our lifetime estimates. 

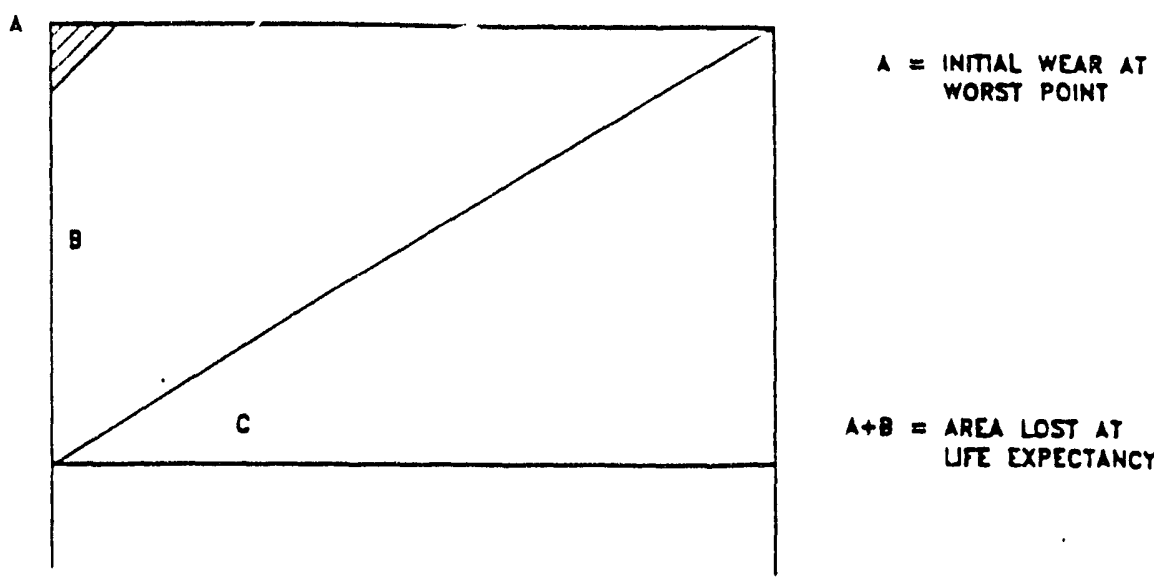

$A+B=A R E A$ LOST AT UTE EXPECTANCY

\footnotetext{
Figure 5. Method of Calculating the Life of a Tungsten Anode
}

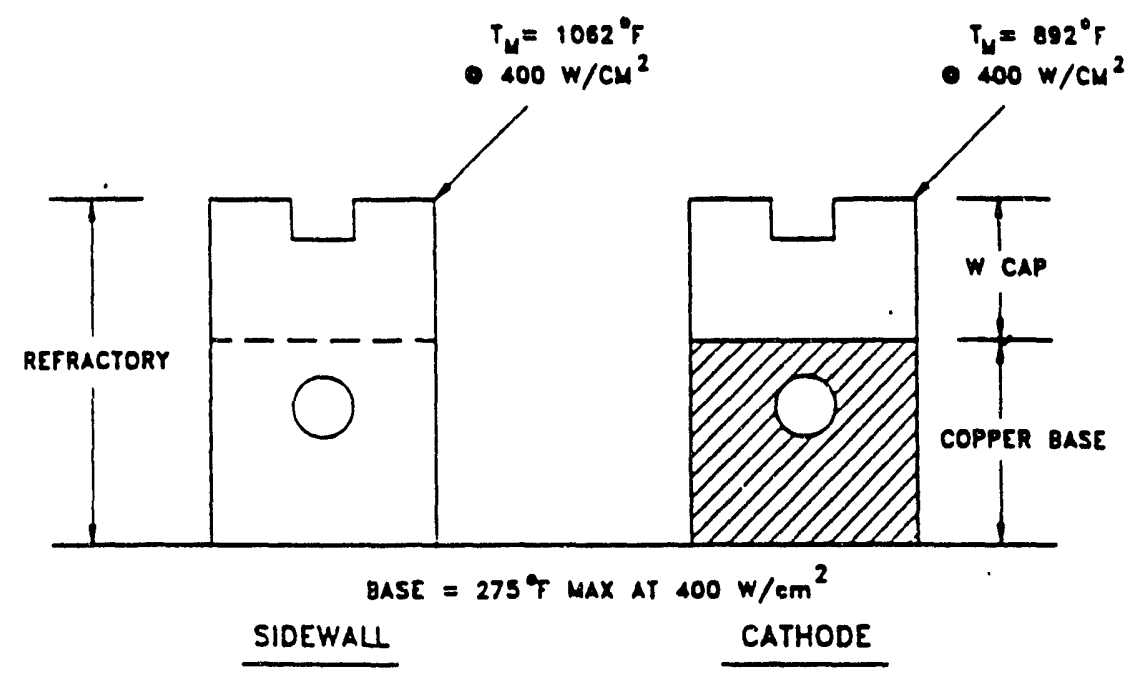

Figure 6. Candidate Cathode and Sidewall Element Design. The dashed line indicates the possibility of a brazed refractory cap on a refractory base. 
TABLE 4

\section{CATHODE WEAR RATES FROM MHD POWER TESTS}

Data includes Mark VII and COIF tests with and without cathode nonuniformities.

COLD COUPONS:

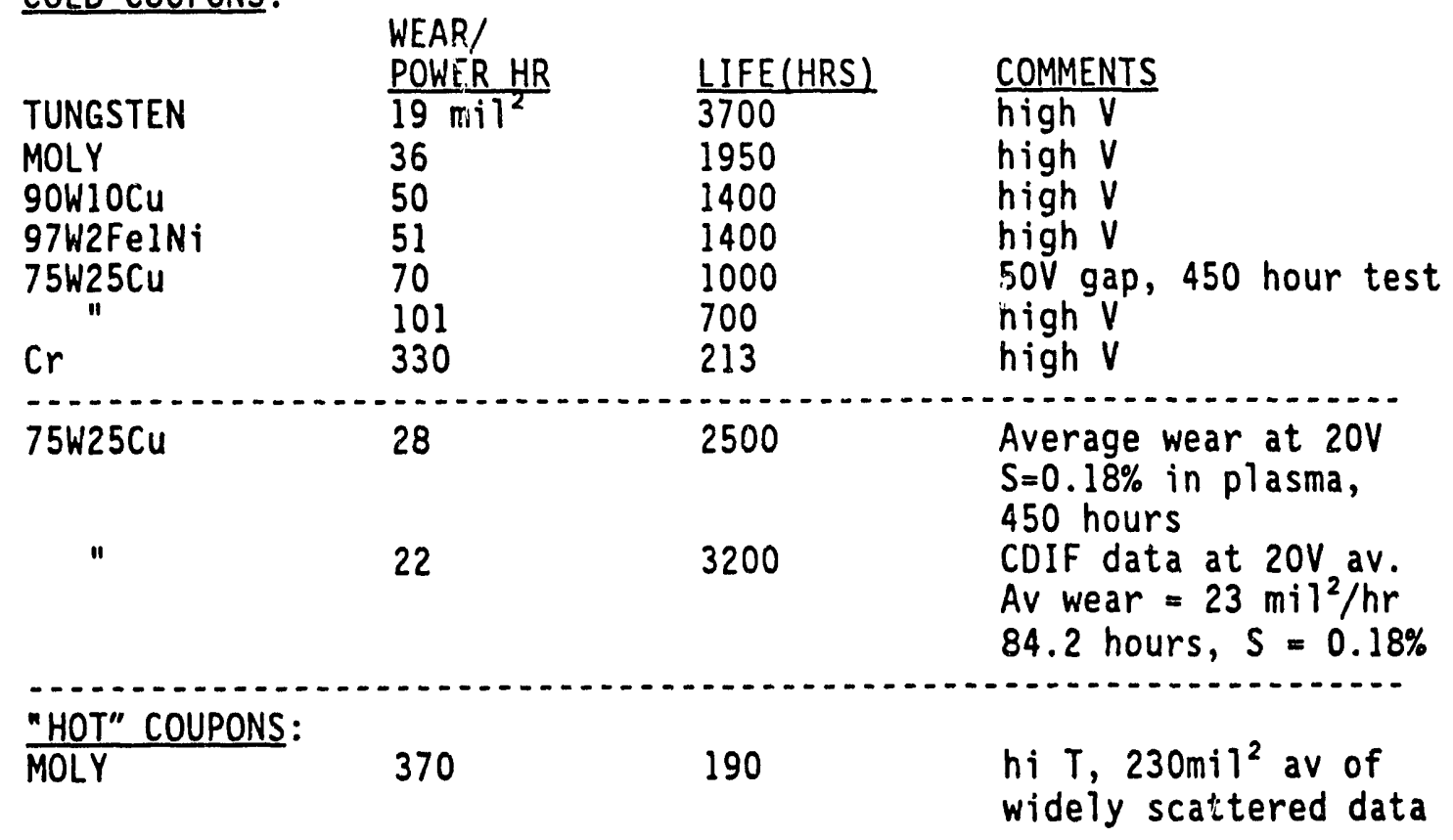

* Worst case data for $j=1 \mathrm{a} / \mathrm{cm}^{2}, S=0.27 \%$ in plasma

Note that the wear of the $75 \mathrm{~W} 25 \mathrm{Cu}$ in the 450 hour Mark VII test was almost $1 / 3$ worse than the wear of $75 \mathrm{~W} 25 \mathrm{Cu}$ in the coal-fired $1 \mathrm{Al}$ test. This contradicts the commonly held misconception that the wear of materials with coal is different from the wear with an ash injected oil combustor. The primary difference between the two generators in terms of cathode wear was the extensive shorting of the cathode wall which took place at the CDIF before the use of iron oxide became standard. In addition the channel hardware originally supplied was not long duration hardware and was intended for facility and power train check-out tests only. The extensive shorting in the 1AI, which occurred before the use of iron oxide, produced high voltage gaps of 150 plus volts characterized by higher wear rates. These latest $75 \mathrm{~W} 25 \mathrm{Cu}$ results were obtained from the $1 \mathrm{Al}$ with the use of iron oxide additive to maintain the gaps at a steady 20 volts with no shorting. The Mark VII data was obtained from gaps which had a 20 volt time average over 450 hours but which in reality were most often around 80 volts open or they were shorted. Thus the 20 volt average is really 80 volts with a $25 \%$ duty factor. The effect of gap voltage on wear can be readily appreciated by comparing the first two (high gap voltage) $75 \mathrm{~W} 25 \mathrm{Cu}$ entries with the second two low voltage gap entries in Table 4. Lifetimes are significantly longer for the low voltage gaps. The higher wear in the Mark VII demonstrates that high voltage gaps wear faster 
by a factor of $1 / 3$ or more depending upon voltage. It also demonstrates that conditions in the Mark VII can be made similar to the high voltage, high wear conditions characteristic of the earlier lAl tests.

The lifetime for a cathode design with a $3 / 8$ inch $W$ cap on a $C u$ base is done similarly to that of the $W$-capped anode shown in Figure 6 except that the wear angle for cathodes is observed to be 45 degrees or higher. Using 45 degrees one obtains a life of 3700 hours. This is the lifetime shown in Table 5 . If iron oxide can be successfully used in the 1A4 POC test, inter cathode voltages will average 25 volts and not the 100-150 volts for the tests on which these wear rates were based. This is an important point. From Table 4 it can be inferred that any of the cathode materi ils tested(except chrome) would last for the requisice 2000 hours if low voltage gaps can be maintained.

TABLE 5

LIFE EXPECTANCY OF W-CAPPED CATHODE DESIGN

(Based upon high voltage gap measurements)

$\begin{array}{ll}\text { DESIGN } & \text { LIFE } \\ 3 / 8 \text { "W over } \mathrm{Cu} & 3700 \text { hours }\end{array}$

\subsection{GAS SIDE CORROSION ANALYSIS}

In the materials test results shown in Table 4 the best cathode material is tungsten, followed by Mo, $90 \mathrm{WlOCu}$, and sintered $97 \%$ tungsten heavy metal alloy. 90Wlocu is too brittle to be safely used as a side bar element, but would make a satisfactory capping material. Its advantage is that it combines a high tungsten content with good thermal conductivity. $75 \mathrm{~W} 25 \mathrm{Cu}$ is not as good a performer on the gas side as the three materials mentioned but it does have two important qualities; high thermal conductivity and more ductility than the other materials. With low voltage intercathode gaps obtained using iron oxide, $75 \mathrm{~W} 25 \mathrm{Cu}$ is an excellent performer.

Thermal conductivity is a very significant factor in cathode wall wear and so the choice of a material for gas side capping and the choice of a material for the water side base, each with its own characteristic thermal conductivity, produces a composite design which operates at a given surface temperature. Two such surface temperatures are shown in Figure 5 . The presence of arcs on the cathode wall, especially leakage current arcs provides two conditions necessary for the loss of material at a cathode gap: the temperature of the metal in the gap is further elevated over and above the non-electrical and the diffuse electrical heat flux, and the upstream edge of the gap becomes anodic, providing electrochemical conditions favorable to the reaction of the metal with seed, slag constituents, iron, and most especially, and perhaps primarily, with $\mathrm{H}_{2} \mathrm{O}$. The other side of the gap is cathodically passivated and therefore stable. Even copper performs well there.

Assume a 50 volt gap carries 30 amps leakage current which dissipates 1500 
watts at the gap. For an electrode with a gas length of $15 \mathrm{~cm}$ and a gap of 0.25 $\mathrm{cm}$, the additional heat flux due to this leakage is $375 \mathrm{~W} / \mathrm{cm}^{2}$, which might be twice the heat flux without leakage. This additional heat flux is localized at the leading edge of the cathode. A 150 volt gap would triple this power figure and the metal at that gap would run quite hot. Microscopically, a one amp arc across a 50 volt gap would dissipate 25 watts at each arc foot. Assuming a 0.25 $\mathrm{mm}$ thermal foot diameter, this means a local heat flux of $50 \mathrm{kw} / \mathrm{cm}^{2}$ at an arc foot. This is not unreasonable since the core of an arc is quite narrow and the plasma temperature well in excess of $5000 \mathrm{~K}$ (A strong arc can have a plasma temperature of $30,000 \mathrm{~K}$.) near the metal surface. Fortunately these arcs are in constant motion and are blown off the cathode wall by the $J \times B$ force. Nevertheless one can appreciate the destructive power of these arcs.

Because it is extremely difficult to model arc behavior it seems reasonable to approach the problem from another, more thermodynamic, point of view. Let us ask the following question: Based upon oxidation tests reported in the literature what average surface temperature is necessary to produce the metal losses in agreement with what we observe in the channel?

Table 6 contains data on the temperature dependence of the erosion of $W$ and data for some additional refractory metals in oxygen containing environments. This data is taken fror results in air and in helium containing $1 \%$ oxygen. At elevated temperatures we assume that the reaction rate of tungsten with water vapor is similar. The refractory metals oxidize rapidiy when exposed to water vapor above $750 \mathrm{C} .{ }^{6}$ Comparing this data with the data in Table 4 will give us an estimate of an average surface temperature of the edge of the cathode which characterizes the wear rate observed in MHD tests. Based upon Jaffe's data, tungsten at $715 \mathrm{C}$ would 10 se about $16 \mathrm{mil}^{2} /$ hour, at $760 \mathrm{C} 32 \mathrm{mil}^{2} /$ hour and at $800 \mathrm{C}$ would lose $64 \mathrm{mil}^{2} /$ hour. On average the wear data is characterized by a leading edge temperature of $715 \mathrm{C}(1320 \mathrm{~F})$. High wear rates characteristic of high voltage, high leakage channels would occur when the surface temperature of the tungsten edge approaches $825 \mathrm{C}(1500 \mathrm{~F})$, according to this oxidation data. It is important to stress here that calculated surface temperatures based on uniform heat flux through a channel wall gives temperatures much lower than these. Therefore to justify observed corrosion rates with observed laboratory oxidation rates one must postulate that there exists a strong nonuniform contribution to the electrode heat flux caused by the nonuniform Faraday and/or leakage current distribution on the electrode walls.

TABLE 6

TEMPERATURE DEPENDENCE OF OXYGEN EROSION OF $W^{7,8.9}$ Data for air and $1 \%$ oxygen in He combined

$\frac{\text { MATERIAL }}{W}$

\begin{tabular}{l} 
TEMPERATURE \\
\hline $1050 \mathrm{C}(1920 \mathrm{~F})$ \\
$825 \mathrm{C}(1500 \mathrm{~F})$ \\
$680 \mathrm{C}(1260 \mathrm{~F})$ \\
$1050 \mathrm{C}(1920 \mathrm{~F})$ \\
$825 \mathrm{C}(1500 \mathrm{~F})$
\end{tabular}

EROSION RATE

$803 \mathrm{mil}^{2} /$ hour

80

8

30,900

Ta $825 \mathrm{C}(1500 \mathrm{~F}) \quad 186$

The question whether the erosion is occurring due to melting and 
vaporization of the tungsten or whether it is due to oxidation has been addressed in the literature. If the tungsten at an arc foot exists at $5000 \mathrm{~K}$ then the evaporative losses would be equal to the corrosion losses. According to Pustogarov et al. ${ }^{10}$ at $3500 \mathrm{~K}$ the evaporative losses are $31 / 2$ orders of magnitude less than the corrosion weight loss. A very shaky extrapolation down to $1800 \mathrm{~K}$ gives an evaporation loss 9 orders of magnitude less. 0xidation would seem to be the more likely phenomenon. The point to be made is that melting and vaporization is not necessary to have the observed wear rates. Lower temperature oxidation is entirely sufficient.

We do not have satisfactory rata for Mo at temperatures lower than $1050 \mathrm{C}$ but Mo is known to be less stable than $W$ so it is not surprising that Mo has higher wear rates than $W$ in Table 4. A capped Mo cathode operates 170F higher than a capped copper base cathode according to the data shown in Figure 6 while a solid Mo cathode would operate over 200F higher. Based upon the activation energy for tungsten oxidation displayed in Table 6 , this temperature difference in Mo would increase the oxidation rate of Mo by a factor of 4 or 5 . In the test data shown in Table 4, there are two entries for Mo: for cold and "hot" coupons. The cold Mo coupon was a $1 / 8^{\prime \prime}$ Mo leading edge brazed to a solid Cu base, while the hot coupon was a solid Mo cathode and the data was based upon about 6 of the cold Mo cathodes and 10 of the solid Mo cathodes. The observed wear rate difference, about an order of magnitude, seen in Table 4 for the two types of Mo cathodes is corroborated by the the oxidation rate difference expected for Mo based upon the admittedly skimpy data shown in Table 6 . A preliminary observation of Tantalum wear is consistent with an average operating temperature of about $690 \mathrm{C}(1280 \mathrm{~F})$. Although one could argue that the temperature at an arc foot and therefore the electrode wear is relatively independent of the non-arc metal temperature, the data for Mo belies this fact since the variation of the non-arc metal temperature is the only explanation we find for the difference in wear between Mo-capped copper and solid Mo cathodes.

Furthermore, molybdenum wear coupons placed elsewhere on the $1 \mathrm{Al}$ channel sidewalls strongly indicate that molybdenum wear rates are nonlinear and decrease markediy as time proceeds. However, because the total test time was only 22 hours, and only a few of these elements were tested, these data were not used in establishing molybdenum element lifetimes. This fact further bears our the definite dependence of wear rate on surface temperature seen in the tests of Mo cathodes in the MK VII. The thinner the Mo element, the colder it operates. Thick Mo elements initially wear faster than $75 \mathrm{~W} 25 \mathrm{Cu}$ elements, but become more durable than $75 \mathrm{~W} 25 \mathrm{Cu}$ as they wear down. It is this effect which gives Mo its long expected lifetime on the MHD cathode in spite of the lower initial wear rate of $75 \mathrm{~W} 25 \mathrm{Cu}$.

We have looked microscopically at a cathode with a Ta leading edge and found evidence which would indicate that oxidation was taking place and no evidence of melting. In addition, in one area the Ta reacted with the EZ Flow 45 braze material at the gas interface causing separation of the braze-Ta interface. This must have elevated the local temperature and been the cause of the high local corrosion there. Otherwise the overall corrosion was low on the average. The $30 \mathrm{mil}$ thick Ta leading edge foil was sufficient to protect the $\mathrm{Cu}$ behind it. Further investigation of Ta on the cathode is worthwhile.

On the anode wall the recession rate of $W$ is given in Table 2 as 73 $\mathrm{mi}^{2} /$ hour. This would correspond to an average temperature of $825 \mathrm{C}$ on the leading edge based upon a comparison of its MHD wear rate to the oxidation wear 
rate data. This is about $110 \mathrm{C}$ higher than the cathode wall leading edges normally appear to operate(again by comparison with oxidation wear rate data), but about the same temperature as the high leakage cathode wall displays through its wear rates. That is, tungsten on the anode wears as if its leading edge were $110 C$ hotter than the $W$ cathode leading edge. This seems reasonable since the anodes have more arc activity than the cathodes because more current transfer between cold, non-slagging anodes and the plasma is via arcs with less diffuse contribution than exists on the cathode. Furthermore oxidizing anions of sulfur and the silicates are driven to the anode from the plasma.

The Anode wall has been shown to be very sensitive to localized heating effects. 62 power hours were accumulated in the anode confirmation test series during summer, 1990. Some of these test hours were at very high current density. In addition, post test photos confirmed the existence of a slag streamer along one side of the anode wall. Therefore most of the Faraday current was collected in this spot due to the localized heating caused by the fact that uneven slagging concentrates the Faraday current.

An axial stringer of slag extending almost the full length of the anode test section caused unusually high current density along that line. This is further evidence that the uneven current density caused by uneven slag accumulation, and its concomitant uneven temperature distribution, leads to uneven wear: in this case along the anode surface. Because of its high surface temperature, a slag covering on an electrode has a much lower boundary layer impedance to charge transfer with the plasma than a much colder metal surface would. Slagging raises the average wall temperature from less than $1000 \mathrm{~K}$ to approximately 1800K. At given gas conditions and normal boundary layer development this is the only way to reduce the size of the Faraday arcs while retaining a "cold" metal temperature. Complete slag layer coverage will eliminate temperature nonumiformities on the wall caused by local slag puddles and/or streamers. These "hot" spots accentuate current concentrations resulting in regions of high localized corrosion. That is, local current densities would be significantly higher where slag is present only on a small part of an anode. During long duration testing slag on the wall develops a uniform covering. In tests characterized by many short runs, the slag layer never has a chance to develop uniformly. As a result, power hours accumulated in short segments (1-20 hours) produce more wear due to slag nonumiformities than do power hours accumulated in large blocks (100-200 hours).

Consider a hypothetical model. If only $10 \%$ of an anode is slag covered then the local current density could be as much as 10 times greater in the slag covered areas than in the unslagged areas. These high local current densities produce localized hot spots on the Pt surfaces. An anode operating at $225 \mathrm{~W} / \mathrm{cm}^{2}$, of which $1 / 3$ is assumed to be electrical power dissipation, with $10 \% \mathrm{slag}$ coverage could have local heat fluxes of $900 \mathrm{~W} / \mathrm{cm}^{2}$ in this model. In this case the local operating temperature (metal temperature) would be quite high, perhaps $1800 \mathrm{~F}$ or $1000 \mathrm{C}$. A metal temperature of $800 \mathrm{C}$ in the MHD environment would sustain an elevated corrosion rate. Clearly the local Pt loss or corrosion rate should depend upon local surface temperature if any chemical co'rosion is taking place at all.

If the corrosion in the Pt were due only to local melting in the region of the arc-foot, then the severity of the effect should be inversely related to the thermal diffusivity of the material and be largely independent of the average local operating temperature since arc foot temperatures are so much larger than 
the normal metal temperatures. Arcs move quickly across the face of an anode so the tracking capability of an arc should be independent of the substrate material under the Pt unless the time average of repetitive arcs in a single track becomes equivalent to the thermal diffusion time. This would be the thermal equivalent of a high localized heat flux due to a slagging nonuniformity.

In summary, analysis of channel gas-side materials wear has served to identify the environmental effects on the various materials tested and to explain anomalously high wear rates when they occur. The sensitivity of cathode lifetime to gap voltage means that under conditions in which iron oxide addition maintains low voltage intercathode gaps, $75 \mathrm{~W} 25 \mathrm{Cu}$ becomes an excellent cathode material. Tungsten is the best cathide material under all conditions, but with high voltage gaps $75 \mathrm{~W} 25 \mathrm{Cu}$ is preceded in durability by Mo and $90 \mathrm{~W} 10 \mathrm{Cu}$.

oxidation (by oxygen or water vapor) is shown to be the strongest candidate for most of the observed refractory metal corrosion on the cathode wall and for the exposed tungsten on the anode wall. This is not to diminish the ability of a strong arc to melt any of these materials, but merely to indicate that oxidation is a very strong effect at temperatures much lower than those for which vaporization plays a role. The strong temperature dependence of oxidation is in agreement with the sensitivity of the corrosion rate to operating temperature and to non-uniform slagging conditions. Observed increases in corrosion are attributed to localized heating at slag non-uniformities and at (arcing) interelectrode gaps. This local heating causes oxidation of tungsten or Mo anodes and cathodes by water vapor and oxygen, and causes grain boundary attack of $\mathrm{Pt}$ anodes. That is, the high temperature oxidation of $\mathrm{W}$ in a high $\mathrm{H}_{2} \mathrm{O}-$ containing environment is consistent with the localized wear seen on the cathode wall. The strong temperature dependent wear seen in Mo is also in agreenient with this effect. The increased grain boundary attack in Pt reported here and el sewhere ${ }^{2}$ in a partially slagged anode wall is also consistent with the effects of localized heating. In this case the reaction is not due to the water vapor but to the chemical species present at the anode wall and to the presence of chemical impurities, especially gold and copper, in the Pt. Those impurities are eliminated by going to high purity platinum. And resistance to grain boundary attack is significantly strengtheiled by using oxide grain stabilized Pt. ${ }^{2}$ Table 7 shows the chemical species present at the surface of a cathode wall making no electrochemical assumptions about the local environment and compares that with those predicted at the anode wall assuming a high oxygen activity there because of the electrochemical changes due to the anodic environment. The anode data was obtained by fixing the oxygen concentration to the value shown, $5.0 \times 10^{-1}$. Since $\mathrm{Pt}$ is attacked by $\mathrm{KOH}$ above $400 \mathrm{C}, \mathrm{K}_{2} \mathrm{CO}_{3}$ above $800 \mathrm{C}$, and by $\mathrm{K}^{2} \mathrm{SO}_{4}$ above $1000 \mathrm{C}$, it is not surprising on the basis ot this table that there is a strong need to keep the Pt temperature low to reduce this corrosion to a minimum. 
TABLE 7

BOUNDARY LAYER GAS CONDITIONS

\section{GAS MOLE FRACTIONS}

NO OXYGEN RELEASE

$$
\begin{aligned}
& P=1 \text { Atm, phi }=0.9 \\
& T=1000 \mathrm{~K}
\end{aligned}
$$

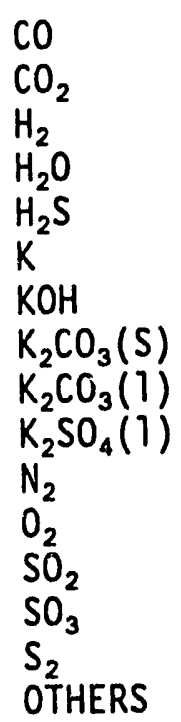

$$
\begin{aligned}
& \text { ANODIC OXYGEN RELEASED } \\
& P=1 \text { Atm, phi }=0.9 \\
& T=1350 \mathrm{~K} \\
& 8.4 \times 10^{-8} \\
& 2.1 \times 10^{-1} \\
& 2.3 \times 10^{-8} \\
& 1.1 \times 10^{-1} \\
& \text { neg1 } \\
& 2.9 \times 10^{-7} \\
& 3.0 \times 10^{-3} \\
& \text { neg1 } . \\
& 7.3 \times 10^{-4} \\
& 1.3 \times 10^{-3} \\
& 1.6 \times 10^{-1} \\
& 5.0 \times 10^{-1} \\
& 2.9 \times 10^{-10} \\
& 2.5 \times 10^{-11} \\
& S=10^{-25}
\end{aligned}
$$

Finally, no microscopic evidence of a developed sulfur corrosion layer in the MHD environment has been seen in analysis of the refractory metals. reaction products in the MHD environment. Evidence of sulfur has been seen, but no dramatic corrosion layer identified. It is well known that sulfur attacks copper anodes and sulfur attack of $75 \mathrm{~W} 25 \mathrm{Cu}$ has been evident in SEM photos. Sulfur, however, is much less reactive with $W$ and Mo than is oxygen and water vapor at elevated temperatures. 


\subsection{WATER CORROSION}

In 1988 waterside corrosion was observed ${ }^{12}$ in $75 \mathrm{~W} 25 \mathrm{Cu}$ sidewall pegs at the CDIF. Upon recent analys is we found galvanic corrosion and some etching near the 0 -ring seal at the water tube end. Inside the waterline there was a copper rich surface, relative to the host, in which there was leaching of tungsten and some pitting corrosion. This peg had only 40 power hours on it but thousands of stagnant hours all at unknown $\mathrm{pH}$ and unrecorded electrical conditions. By comparison, a copper peg, not necessarily from the same electrical environment, showed general corrosion and pitting characteristic of erosion corrosion due to the high velocity water. $\mathrm{pH}$ was not monitored at the time, but subsequent measurements demonstrate a clear likelihood of swings in $\mathrm{pH}$ down to 5 . This would depend upon operating pricedures and on the state of the resin bed. Normal cu usage is recommended, $t \mathrm{pH} 9$. Evidence of high velocity effects was in the patchy way the protective film came off. High velocity polishing of the $\mathrm{Cu}$ surface, while it removes the protective oxide film, also maintains good thermal contact between metal and coolant, thus lowering the metal interface temperature. Minimum corrosion of copper occurs near pH 9. Cross sectioning the specimen revealed that most of the pits were on the wall side, not the heat flux side. Temperature does not appear to aggravate the situation. Close examination clearly showed a 5 mil reddish copper oxide corrosion layer. This kind of oxide corrosion can be induced in copper when made a cathode in an acidic environment. other end pegs showed a patchy greenish discoloration on an otherwise black tungsten oxide background. The black tungsten oxide film was reproduced in subsequent bench tests in which tungsten copper was run as an anode. The origin of the greenish discoloration appears to be due to stagnant, oxygenated water conditions reacting with copper and producing the commonly observable greenish malachite scale on drying.

Pits observed in the $75 \mathrm{~W} 25 \mathrm{Cu}$ water hole inner surface are a common form of corrosion due to leaching of the host into deionized water. None of the observed corrosion was deemed to be life limiting with the possible exception of the pitting in the 75 W25Cu 0 -ring seal area. However this would not be a problem on a bar sidewall which has no 0-ring seals and which therefore has greatly reduced electrochemical currents due to the longer distances between elements. Refractory metals are attacked slowly in basic solutions but are relatively stable in acidic solutions. ${ }^{13}$ Therefore it was recommended that the $\mathrm{pH}$ be maintained at 6.5 or 7 for a balance between the needs of $\mathrm{Cu}$ and of $W$. In addition, high heat flux tests were deemed necessary to determine if the corrosion layer on the $75 \mathrm{~W} 25 \mathrm{Cu}$ will significantly elevate the metal temperature since no comparable experience could be found within the water treatment industry.

\subsubsection{WATER CORROSION TEST RESULTS}

Bench scale water corrosion tests of $75 \mathrm{~W} 25 \mathrm{Cu}$ at AVCO demonstrated excellent performance at $\mathrm{pH} 7$. These tests were performed at ambient dissolved oxygen (3-4 $\mathrm{ppm})$ to simulate CDIF conditions even though power plant conditions call for almost 1000 times less dissolved oxygen (DO). ${ }^{14}$ Water corrosion of Mo is similar to $W$ and is dependent upon the level of DO. Isothermal bench tests with Mo at $100 \mathrm{C}$ and no DO showed no corrosion in 2000 hours. ${ }^{15}$ Therefore the lack of standard power plant DO control at the CDIF places $75 \mathrm{~W} 25 \mathrm{Cu}$ at a distinct 
disadvantage for the POC test. Furthermore, bench scale tests showed a marked deterioration in the performance of $75 \mathrm{~W} 25 \mathrm{Cu}$ at $\mathrm{pH} 5$ in contrast to the excellent results at $\mathrm{pH} 7$. $\mathrm{pH}$ control will not be implemented for the $\mathrm{POC}$ test and its absence may further penalize the performance of $75 \mathrm{~W} 25 \mathrm{Cu}$ since past experience shows that the CDIF water fluctuates between $\mathrm{pH} 5$ and 6.5 . For this reason it has been difficult to reproduce the excellent bench-scale waterside performance of $75 \mathrm{~W} 25 \mathrm{Cu}$ in real-time tests at the CDIF.

A high heat flux test was performed for 75 hours of exposure to water at $\mathrm{pH} 7$ with one megohm resistivity. Water temperature was maintained at 140-160F under an oxyacetylene torch which developed a heat flux of $400 \mathrm{~W} / \mathrm{cm}^{2}$ to simulate the MHD channel environment under power. In addition the water velocity was maintained at $30 \% / \mathrm{sec}$ and the test specimen was held at 100v anodic to sustain any possible electrochemical reactions for complete replication of channel conditions. Flow conditions produced a metal temperature of $400 \mathrm{~F}$ in contact with the water.

Upon completion of the test the specimen was sectioned and studied using scanning electron microscopy (SEM) and scanning Auger microscopy (SAM). On the top surface of the water hole, the hottest region, there was a black surface film of $W$ metal with $30 \%$ oxide and which was electrically conducting. This film was about 600 Angstroms thick and declined in oxide content to less than $5 \%$ as one progressed deeper towards the metal surface. The thinness of the film as well as its primarily metallic makeup mean that the film is not a barrier to heat transfer. In addition during the test there was no significant increase in the metal temperature of the peg within the resolution of the experiment. This was further indication that a thermal resistance layer did not build up on the surface of the water cooling passage.

On the bottom (cooler) surface a similar film was observed but there were al so some tubercular growths, about 1000-10,000 Angstroms thick, and which were mostly metallic consisting of $\mathrm{W}+\mathrm{O}+\mathrm{C}$ located only on the machine stress marks. These too were not deemed to endanger heat transfer, especially since they were on the bottom of the water passage.

Corrosion was too low to be measureable or to be life threatening: the water hole did not increase in size to the one mil resolution of the measurement. In fact machining marks were still visible.

While we do not believe that uncontrolled water for the POC test will be life-limiting for $75 \mathrm{~W} 25 \mathrm{Cu}$, a deterioration of the performance could cast a misleading doubt on its usefulness as a waterside material for the next generation channel scale-up. Additional corrosion on the waterside could produce additional thermal resistance for heat transfer to the water. In that case the resulting increase in gas-side operating temperature will produce additional and unnecessary wear of the gas-side surfaces as evidenced in the temperature dependence of wear in Mo and $W$. It is well to keep in mind that 75W25Cu is not an al loy and the sintered grains of dissimilar metals can easily set up corrosion couples under the right conditions.

The high visibility green-colored corrosion layer which can develop in the 75 W25Cu water passages does so when the passages air dry under dirty conditions. Repeated $(25 x)$ air drying with tap water showed little evidence of the greenish corrosion layer. 6 months continuous underwater exposure to an aqueous environment with ambient DO produced little surface corrosion (some slight blackening). The test was done in the absence of other materials whose presence might produce corrosion couples between the dissimilar materials. However the 
$\mathrm{pH}$ decreased from 7 to 3.5 during the test so stagnant water conditions represent an uncontrolled corrosion environment, even with water initially of $\mathrm{pH} 7$. A standing channel contains dissimilar materials and corrosion couples may occur.

On the basis of these tests we have set the water $\mathrm{pH}$ range to be 6.5-7.5 for acceptability for POC test conditions. Since corrosion has been observed in 75W25Cu both in Mark VII and IAI channel tests when pH was uncontrolled, we feel certain that $\mathrm{pH}$ is the critical parameter defining acceptable performance of the material. However controlling the dissolved oxygen content of the cooling water within acceptable power plant limits is standard industry practice and cannot be ruled out.

\section{WATER CORROSION: CONCLUSIONS}

1. $\mathrm{pH}$ is the critical factor affecting performance of $75 \mathrm{~W} 25 \mathrm{Cu}$. Low $\mathrm{pH}$ is very likely to be the cause of the corrosion of $75 \mathrm{~W} 25 \mathrm{Cu}$. Recommend CDIF set $\mathrm{pH}$ at 6.5-7.5

2. Increased heat flux appears to reduce the deposits in the observed corrosion.

3. While there is no confirming evidence from AVCO bench-scale tests to back this recommendation, standard power plant practice of DO control is recommended in the range of 50-200 ppb. Isothermal bench tests with Mo at $100 C$ and zero DO showed no corrosion in 2000 hours.

4. Stagnant water in contact with dissimilar metals can produce large changes in $\mathrm{pH}$. Such conditions may produce elevated galvanic corrosion and thus should be avoided in the IA4 channel.

5. It is important to record $\mathrm{pH}$ levels during the POC test series as well as to $\log$ the wet vs. dry down time for the channel. The water passages should be monitored periodically to warn of any deterioration so proper $\mathrm{pH}$ and $\mathrm{DO}$ controls can be instituted before serious damage occurs.

\subsection{CONCLUSIONS}

The work performed to date in the selection and testing of channel gas side materials has served to identify and clarify the respective environments to which the various materials are subjected and identified a performance ranking of materials to be folded into the general class of manufacturing issues which must take into account all the other issues besides gas side wear. We have shown that the prospective anode designs should meet the 2000 hour lifetime requirement based upon MHD materials tests. Cathode materials have been ranked according to their relative survivability in the MHD environment. For high voltage intercathode gaps, which show the most severe wear, the materials rank in the order from best to worst: W, Mo, 90W10Cu, 97W2FelNi, $75 \mathrm{~W} 25 \mathrm{Cu}$, and $\mathrm{Cr}$. We have shown data which indicates that lifetime is sensitive to gap voltage. Therefore for conditions under which iron oxide addition maintains low voltage intercathode gaps $75 \mathrm{~W} 25 \mathrm{Cu}$ becomes an excellent cathode material. Finally oxidation is shown to be the strongest candidate for most of the observed corrosion on the refractory-capped cathode wail and for the exposed tungsten on the anode wall. This is not to preclude the ability of a strong arc to melt any of these materials, but merely to indicate that oxidation is a very strong effect at 
temperatures much lower than those for which vaporization plays a role. The strong temperature dependence of oxidation is in agreement with the sensitivity of the corrosion rate to operating temperature and arcing conditions. It is well known that sulfur attacks copper anodes ${ }^{18}$ and sulfur attack of 75 W25Cu has been evident in SEM photos. There are enough questions remaining concerning the behavior of these materials that before a commercial MHD channel can be designed and fabricated further testing and evaluation of materials will be necessary. These tests must be done under electrical and gas side conditions characteristic of the scale-up channel(especially with respect to interelectrode voltages, current densities, and boundary layer and current leakage effects). Also, explorations into non-shorting cathode designs and slagging anode designs are important issues for the next generation channel.

On the water side $\mathrm{pH}$ is critical factor in performance of $75 \mathrm{~W} 25 \mathrm{Cu}$. Low $\mathrm{pH}$ is very likely to be the cause of the corrosion of $75 \mathrm{~W} 25 \mathrm{Cu}$. It is recommended that the $\mathrm{pH}$ be set at 6.5-7.5. While there is no confirming evidence from AVCO bench-scale tests to back this recommendation, standard power plant practice of DO control is recommended in the range of 50-200 ppb. Isothermal bench tests with Mo at $100 \mathrm{C}$ and zero DO showed no corrosion in 2000 hours. Stagnant water in contact with dissimilar metals can produce large changes in $\mathrm{pH}$. Such conditions may produce elevated galvanic corrosion and thus should be avoided in the lA4 channel. It is important to record $\mathrm{pH}$ levels during the POC test series as well as to log the wet vs. dry down time for the channel. The water passages should be monitored periodically to warn of any deterioration so proper $\mathrm{pH}$ and DO controls can be instituted before serious damage occurs. 


\subsection{REFERENCES: CORROSION}

1. R.J. Pollina, W. Simpson, and L.C. Farrar, MHD Channel GAsSide Element Erosion- Corrosion Studies, SEAM 28, Chicago, 1990.

2. R.J. Pollina et al., in " $1 A 4$ Materials corrosion and Confirmation Test Results, SEAM 29, New Orleans, LA, 1991

3. R.J. Rosa and R.J. Pollina, "Corrosion and Arc Erosion in MHD Channels", Conference of DOE University Coal Research Projects Pittsburgh, PA, June 25-27, 1991.

4. R.J. Rosa and R.J. Pollina, DOE University Coal Research Program Review, Knoxville, TN, September 27, 1990.

5. L.C. Farrar, "Coal-fired $1 A 1$ channel gas-side element designs and materials test results from the CDIF", SEAM 28, Chicago, June, 1990.

6. Philips Elmet Co., private communication.

7. Jaffe, Properties of Refractory Metals, in Problems in Modern Metallurgy, 54, 6, 115-141(1960).

8. The tungsten data of reference 6 is in agreement with evaporation data for tungsten in oxygen-containing rare gases of A.V. Pustogarov et al., "Effect of oxygen inpurities in helium and argon on the operation of tungsten cathodes.", Teplofizika Vysokikh Temperature 27, 792-796(1968).

9. This data is also in agreement with the data shown for Mo in Figure 7.7 and that shown for $W$ in Figure 7.10 in "High Temperature oxidation of Metals", by Per Kofstad, John Wiley and Sons, NY, 1966.

10. A.V. Pustogarov et al., "Effect of oxygen inpurities in helium and argon on the operation of tungsten cathodes.", Teplofizika Vysokikh Temperature 27, 792-796(1968). 
11. Herbert H. Uhlig, "The Corrosion Handbook", John Wiley and Sons, NY, 1948 .

12. Ron Glovan, "Performance of Cu-W Materials in MHD Service", MSE internal memo, February 5, 1988.

13. Refractory metals may be etched in basic solutions with the assistance of applied electric current. This electrochemical etching procedure is used in the industry to produce an enriched $\mathrm{Cu}$ surface on Tungsten copper alloys before brazing. similarly, because of the stability of refractory materials to strong acids, electrochemical acid etching can be used to produce a lustrous refractory-metal-rich surface by removing the copper from tungsten copper alloys.

14. EPRI Report CS 4629, Interim Consensus Guideline on Fossil Plant Cycle Chemistry, June, 1986.

15. J. Electrochem. Soc. 118, 443 (1971).

16. V Hruby et al., SEAM 19. 


\section{CURRENT DISTRIBUTION; INTRODUCTION}

In the discussion of corrosion the strong dependence of the rate of corrosion upon temperature has been described. In the MHD environment the surface temperature at the slag-metal interface is in turn a strong function of the electric current distribution. The location and rate of transport of corrosion causing agents to the surface and the rate of electrolytic corrosion processes is also strongly influenced by the current distribution. Accordingly a study has been made of the current distribution in slag and it's effects. This includes the examination and analysis of data from Avco and CDIF tests.

Studies are made of two components of current. The first is the transverse or "Faraday" current, which flows from the plasma through the slag layer to the electrodes. The second component of current, and perhaps the most important for corrosion, is the current leak driven by the Hall voltage axially through the slag layer. This axial current may also significantly reduce power output.

Axial leakage current is difficult to measure directly. Spacial and temporal variations tend to cloud the interpretation of local measurements such as the current or voltage between any two neighboring electrodes. Also the measurement process itself tends to affect the result in an unpredictable manner.

Because axial leakage current is difficult to measure directly several methods for inferring it from the other data routinely collected during a run have been developed. These methods have been applied to the analysis of a number of CDIF runs. Inferred leakage current in some cases turns out have a value of from 100 to 200 amperes. This is enough to have a major impact on both power and corrosion.

It has also been found that apparent leakage current is a strong function of Faraday current while only weakly dependent upon axial field. Possible reasons for this unexpected result and the insight that it gives into leakage mechanisms are discussed.

Attempts to directly measure axial leakage current or infer it indirectly from data routinely measured during CDIF operation have been made by Pian, McLain, and Schmitt (1), Daniel and Lohrasbi (2), Nel son, Lee, Stephan, Wu and Lineberry (3), and the principal investigator (4). Indirect methods basically consist of putting measured quantities such as jy, Ey, and Ex into the MHD equations, calculating what the diagonal load current should be, and assuming that leakage current must be the difference between the calculated current and that actually measured. All investigators have inferred leakage currents in the range of 100 to 200 amperes.

An important input required when employing indirect methods, and one difficult to obtain, is the conductivity of the plasma at the time when the other data was taken. In the following section a method is described for inferring the value of the gas conductivity under load and the resulting leakage current. Inferred leakage current for nearly all of the CDIF runs made during the past several years is presented and correlated. These include one oil fired run, two Farady loaded runs, and many diagonally loaded runs, some with iron oxide added over the electrodes and some without. Also a few cases early in a run before polarization had occured are analized. Leakage is also presented as a function of axial location in the channel.

In order to enhance interpretation of the results, two additional methods for inferring leakage and other properties in the generator have been employed in this study. These are also described below and the results summarized and evaluated. There then follow recommendations for further work. 


\subsection{FARADAY VOLTAGE PROFILE; METHOD ONE}

This indirect method consists in essence of inserting measured quantities such as $j y, E y$, and Ex into the MHD equations, calculating what the diagonal load current should be, and assuming that leakage current must be the difference between the calculated current and that actually measured. All investigators using this or similar methods have inferred leakage currents approaching or sometimes exceeding 100 amperes (Refs. 1,2,3,4,5). Daniel et al note that leakage current appears to be a maximum at the point in the channel where link current is maximum, not where one might expect it to be, namely at the point of maximum axial field.

To carry out this method it is necessary to know what the conductivity of the gas is, or else to limit the analysis to a location in the duct where axial voltage gradient, Ex, is zero as was done by Nelson et al. However this severely limits the utility of the method. In order to analyze other locations, Daniel et a) assume that conductivity is equal to that measured experimentally before power generation. This also is a limitation, the reasons being the following. Mach number and hence conductivity changes appreciably under load. Al so not all power runs are preceded by a conductivity measurement, and burner output conditions often change significantly from run to run and during a run in spite of attempts to keep burner inputs invariant.

In the work carried out under this contract a procedure is used for inferring the value of the gas conductivity while under load for each run analyzed (Refs. 4,5). This procedure is based upon the Faraday voltage profiles routinely measured at five locations in the CDIF duct and is a key part of this method of analysis.

Conductivity and inferred ieakage current for nearly all of the CDIF runs made during the past several years have been determined and correlated. These include one oil fired run, two Faraday loaded runs, and many diagonally loaded runs, some with iron oxide added over the electrodes and some without. Al so a few cases early in a run before polarization had occurred are analyzed. Leakage is also presented as a function of axial location in the channel.

\section{Description of the Method}

Assuming that the difference between predicted and measured load current must be the leakage current, the equation for the leakage current is;

$$
I(\text { leak })=Y * Z *\{(B+\Phi)|j y|-\langle\sigma\rangle|E x|\}-I(\text { load })
$$

where: $\Phi \equiv$ diagonal angle.

$$
\begin{aligned}
& B=\text { Hall parameter } \\
& \sigma=\text { conductivity } \\
& j y=\text { transverse or "Faraday" current density } \\
& E x=\text { axial or "Hall" electric field } \\
& I=\text { current }
\end{aligned}
$$

and $Y$ and $Z$ are the height and width of the duct (magnetic field parallel to the $z$ axis). All quantities are averaged over the width $Z$, and quantities in brackets, $\langle>$, are also averaged over $Y$.

With one exception, all the quantities on the right hand side of Equation. 
1.1 are known or routinely measured. The quantities $Y, Z$, and $\Phi$ are channel design parameters. The Hall parameter, $B$, can be calculated from measured (or calculated) pressure and known magnetic field strength with reasonable certainty. The Faraday current density can be inferred from the measured current per electrode with some uncertainty (probably small) because of possible leakage in the Faraday direction. The axial electric field can be calculated from measured voltages along the anode and cathode walls. Some uncertainty is introduced in regions where (smoothed) anode and cathode voltage gradients differ, i.e. in the fringe fields at the ends. In the ends the relation between current density and electrode current is also uncertain. However, for most of the duct this should not be a problem.

The major uncertain quantity is the average conductivity $\langle 0\rangle$. This is true, even if it has been measured, for reasons noted above. Therefore it is important to have a way to deduce $\langle\sigma\rangle$ under load at any time desired.

When power is being generated it is impossible to make any measurement that unambiguously yields a value for $\langle\sigma\rangle$ (The best one can do is get a value for $\langle\sigma\rangle$ $+\sigma x$ where $\sigma x$ is the effective axial leakage conductivity). However, an upper and lower limit can be deduced. In the process one also gains information about the uniformity of the gas.

First one obtains the transverse resistivity, $r$ (or $\langle 1 / \sigma\rangle$ ), from one electrode wall to the other. If we defined it so as to include all effects of the boundary layers and any other plasma nonuniformities, it can be calculated from Ohm's law as follows,

$$
r \equiv\langle 1 / \sigma\rangle=\frac{\langle U B\rangle-\langle E y\rangle+B E x}{(1+B \star B) j y}
$$

where $\langle E y\rangle \equiv$ (cathode $V$ - anode $V$ )/(Distance between anode and cathode walls), and all terms in this and the following equations are defined so as to have positive values. (Strictly speaking $\langle r(1+B \star B)\rangle$ is measured, but the error introduced by using Equation 1.2 is small. Generally $B$ is an average based upon an assumed boundary layer thickness).

Secondly, at locations where a transverse voltage distribution has been measured the core conductivity can be deduced from the following,

$$
\sigma c=\frac{\left(1+B_{c}{ }^{\star} B_{c}\right) j y}{u_{c} B+B_{c} E x-d V / d H}
$$

where $\mathrm{dV} / \mathrm{dH}$ equals the voltage obtained by extrapolating the centerline electric field to the walls divided by the distance between walls (equal to $Y$ in Eqn. 1.1). It is routinely presented in the CDIF data reports, on $\mathrm{Pg} 11,12,13$ of the spatial plots at several electrode locations. The subscript c implies core values.

The value of the average conductivity under load must lie in between the core conductivity and the reciprocal of the transverse resistivity, $1 / r$. Generally, when analyzing CDIF data, the percentage difference between the two is in the range of 10 to $20 \%$. Therefore if we take the arithmetic mean, 


$$
\langle\sigma\rangle \approx((1 / r)+\sigma c) / 2
$$

we should usually obtain a value for average conductivity accurate to better than \pm 10 percent. As a check, values inferred by this method are compared to measured values in figure 1.1. If burner conditions remain identical the value inferred under load at electrode 139 should lie above the no-load value there, but likely be below the no-load value at an electrode well upstream such as No. 40. It can be seen that this is pretty much what happens.

The nondimensional product of equations 1.2 and 1.3, namely,

$$
r * \sigma(\text { core })=\frac{\mid\langle U B>|+B| E X|-|\langle E y>|}{\mid\langle U B>|+B| E x|-| d V / d H|}
$$

is of interest for two reasons, the first being as a consistency check. If the data and calculations are consistent and error free the product should have a value greater than but reasonably close to unity. Typicaliy it is found to have a value in the range of 1.1 to 1.2 , and this accords fairly well with what is expected on the basis of arc-boundary layer calculations.

The second reason for interest in this product is that it (or more precisely the difference, $(r \star \sigma c-1))$ is a measure of plasma nonuniformity.

For clarity, the equations presented above are simplified versions of the equations actualiy used. The latter, which include effects such as transverse current leakage and surface voltage drop, are presented in Appendix $A$.

\section{Results}

The results obtained by applying the above equations are summarized in the following figures. Figure 1.2 shows a typical example obtained during a run which included a load sweep. Inferred local leakage current is plotted vs measured local axial field at several positions down the channel. Excluding the data from the furthest upstream electrode, a line drawn through the data has a slope corresponding to a resistance per frame of $0.45 \mathrm{ohms}$ and at zero field an intercept of 95 amperes.

It has been found that the intercept tends to be smaller during runs where iron oxide is injected. However it has been commonly observed that power output and leakage at optimum loading ( $V x \approx 4500$ volts) do not greatly change when iron is added.

The relatively weak dependence upon iron injection (and the number of open gaps) is one mystifying feature of these curves. Others are the non-zero intercept at $E X=0$, and the dependence on the value of gas conductivity. It is strange that leakage through the slag, if that is indeed what we are observing, appears to depend upon the properties of the gas but not on the composition of the slag. Possibly leakage should be plotted vs something else.

It turns out, indeed, that a good way to correlate the results of different runs is to plot leakage vs Faraday current, jy (a few plots vs $\langle\sigma\rangle$ have been made and they look essentially the same). For pre-Aug. 1989 runs Fig. 1.3 is the result. All of the points on this figure are for electrode 139 and a mid-range diagonal load voltage of about 4500 volts.

Most of the data are from $r i: s$ during which no iron was injected. This data seems to roughly define a curve suggesting either a jy squared dependence or a first power dependence with a non-zero intercept on the horizontal axis. Data 
Conductivity (Mho/m)

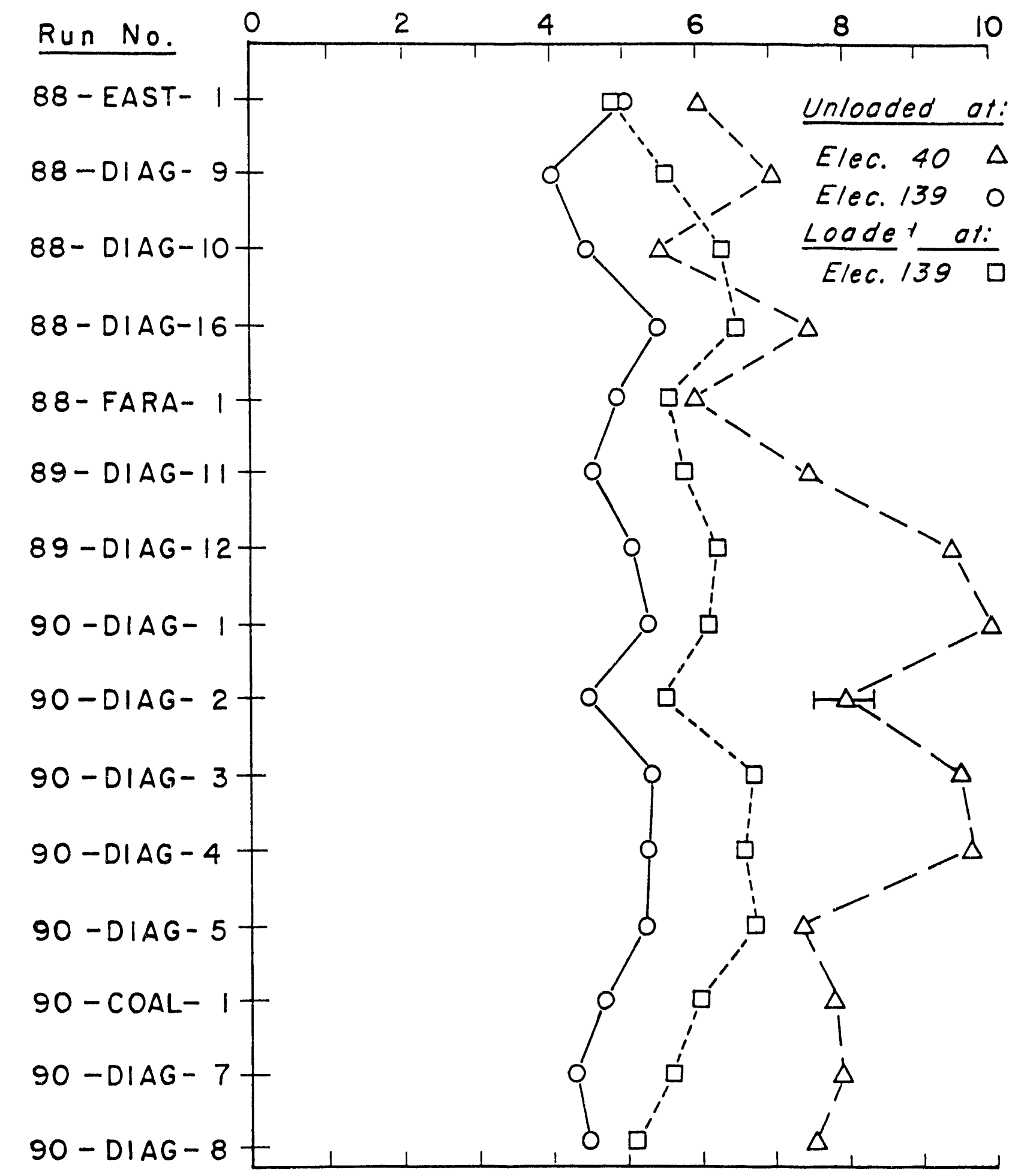

Figure 1.1 Comparison of inferred conductivity under load with measured conductivity while unloaded. 


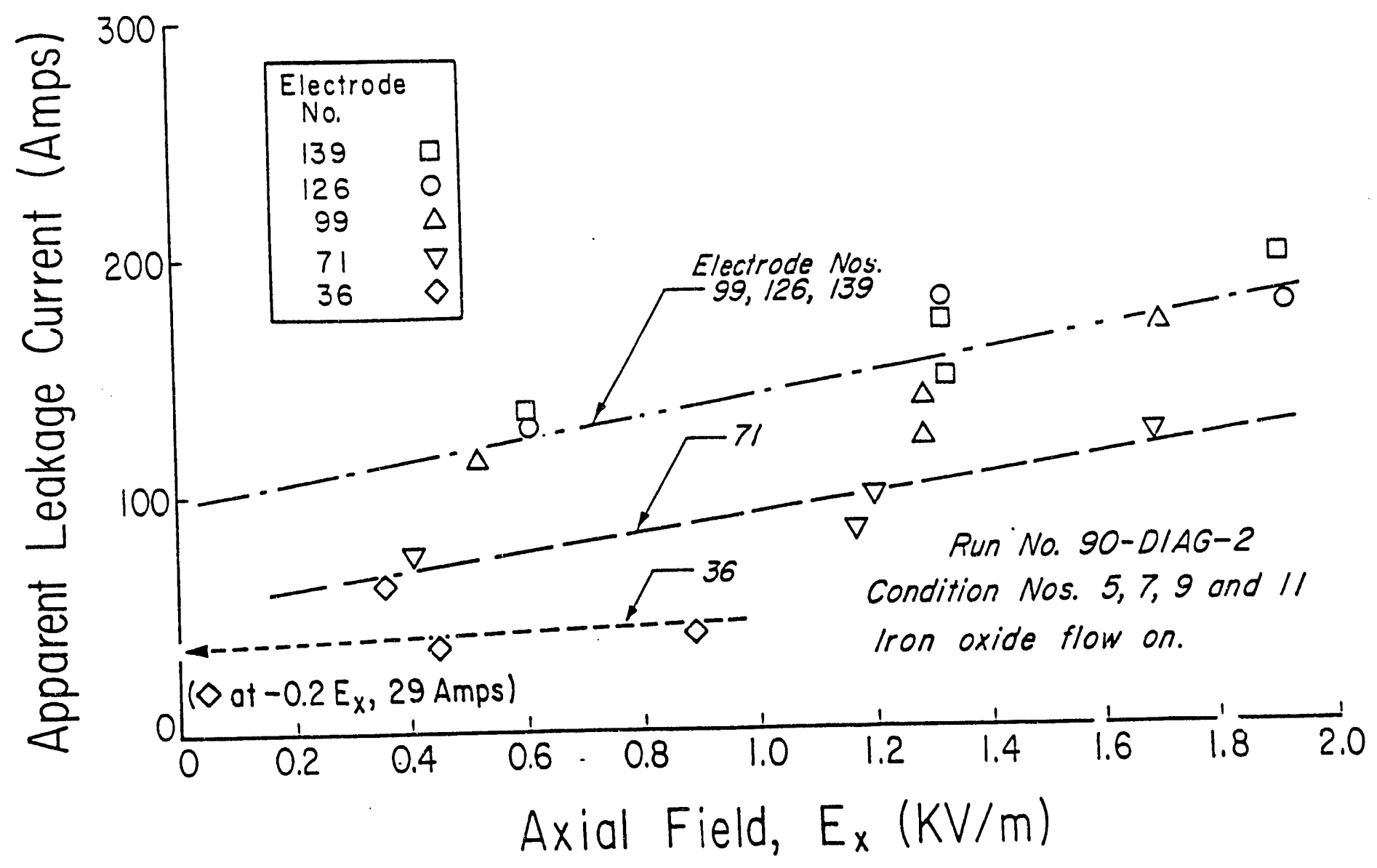

Figure 1.2 Typical plot of apparent leakage current vs axial field obtained during a load sweep. Condition numbers correspond to those in the Preliminary Data Report issued by MSE. 
from short runs or data taken early in a run before the slag polarized and before iron was added tend to fall below the curve. Eastern coal runs also exhibit somewhat smaller leakage.

The following figures show data from more recent runs, specifically 89DIAG-4 and later. Figure 1.4 shows leak current plotted as a function of $j y$. This is again for electrode 139 but all values of Ex. All the data pretty much line up, with a scatter that is of about the same order as the accuracy with which jy can be read off the CDIF spatial plots. Moreover it doesn't seem to matter what Ex is, except for the essentially accidental fact that the lowest and highest values of Ex have been measured when jy was lowest and highest. The two high points for run 90-DIAG-12, along with other data from this run, indicate that something unusual was going on which did not happen in a subsequent long run.

The effect of axial position is shown by figure 1.5 where leak current is plotted vs jy for 5 different locations during run 90-Elec-2. Instead of one curve we now have five -more or less, therefore some position-dependent factor must be important. One possibility is $B$.

Figure 1.6 shows the data from $90-E l e c-2$ plotted $v$ s the product (Bjy), for all electrodes and all Ex. Remarkably, this does a pretty good job of condensing the 5 curves into one. Going a step further, figure 1.7 shows the same data plus data from run 89-Diag-12 plotted vs (Bjy) ${ }^{2}$. Now the curve has become a rather good approximation to a straight line through the origin.

The data scatter does not allow the power of $B$ to be determined with much confidence, however. It will be argued below that, at least for the CDIF, the duct width, $Z$, works as well as $B$ as a correlation factor, and may be easier to explain.

In an attempt to clarify the effect of the slag layer some of the old data were re-analyzed. In particular, leakage values at electrode locations other than 139 have been obtained, the object being partly to look for effects of position, and partly just to improve the statistics by getting more data points.

All electrodes for which transverse profiles are measured were analyzed for six runs. Two of these had iron injection, and four had none. Of the four, one was oil fired (AIC). The other exhibited no polarization, even though there was no iron and the coal was Western. This remarkable and useful fact was evidently due to the data having been obtained after only 90 seconds of operation under power. Also the burner had only been operating for about 20 minutes, so slag coverage may have been incomplete.

The results, plotted as apparent leak vs ( $p t * Z * j y)$, are shown in Fig. 1.8 . Here $p t$ is the electrode pitch, $Z$ is the width of the electrode wall, and jy is the Faraday current density, jy. Thus the product is simply the current per electrode. This product is chosen because it does quite a good job of collapsing data from different axial locations into a single line. In figures 1.6 and 1.7 the Hall parameter, $\beta$, was used instead of $Z$. Since $\beta$ is proportional to pressure and since in the CDIF duct geometry pressure is nearly proportional to $Z$, it turns out that either $\beta$ or $Z$ correlate the data about equally well. However, the latter now seems more in accord with what are believed to be the dominant mechanisms creating the apparent leak. This point will be discussed later.

In figure 1.8, which includes data from all electrode locations where transverse voltage profiles are measured except \#36, it's reasonably evident despite the scatter that most of the "Fe OFF" data lies above the "Fe ON" data, while the "No polarization" and "AIC" data lie below. Therefore the state of the 


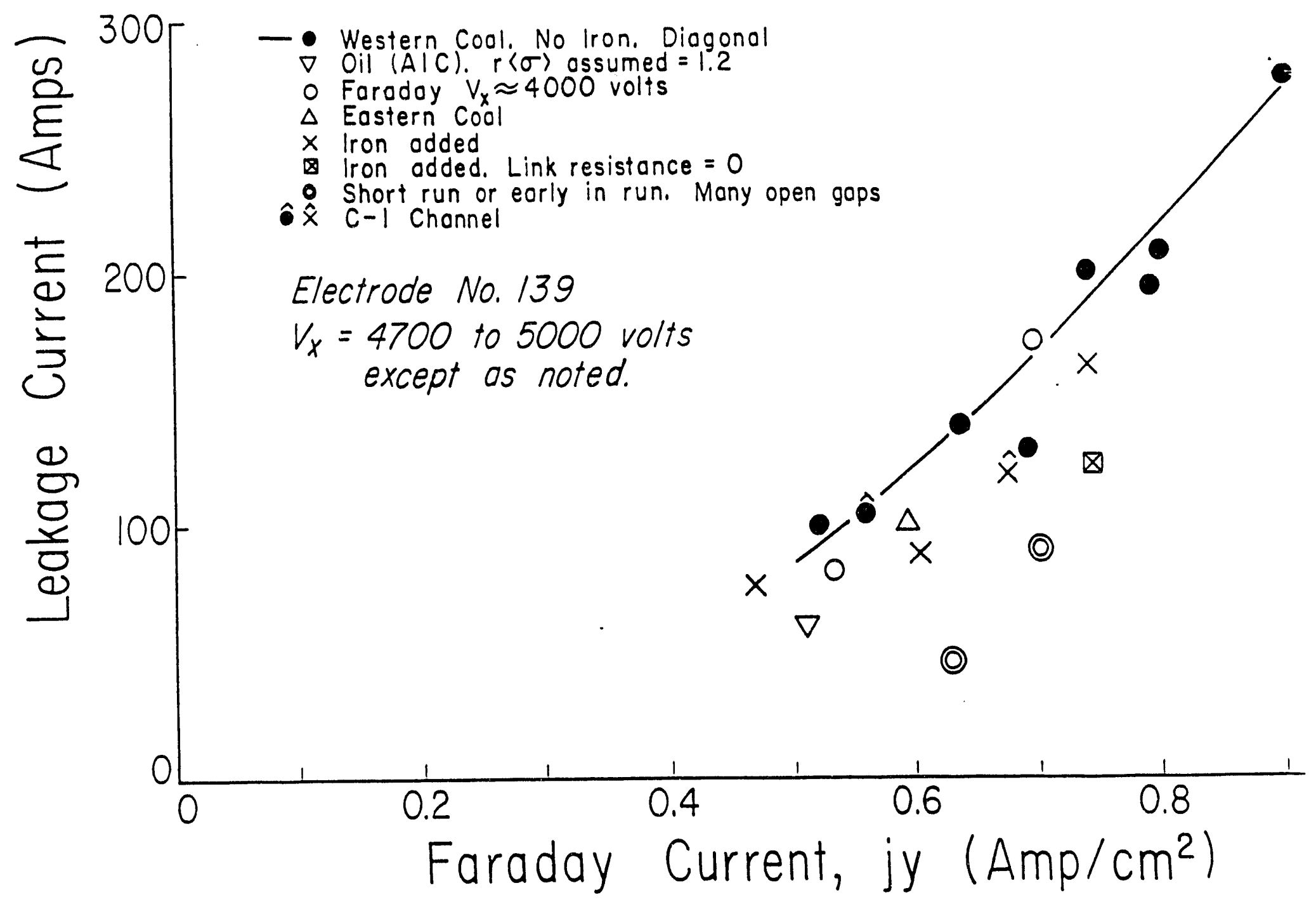

Figure 1.3 Apparent leak current vs measured Faraday(transverse) current density for runs prior to and including 89-DIAG-4, all for approximately the same axial electric field. 


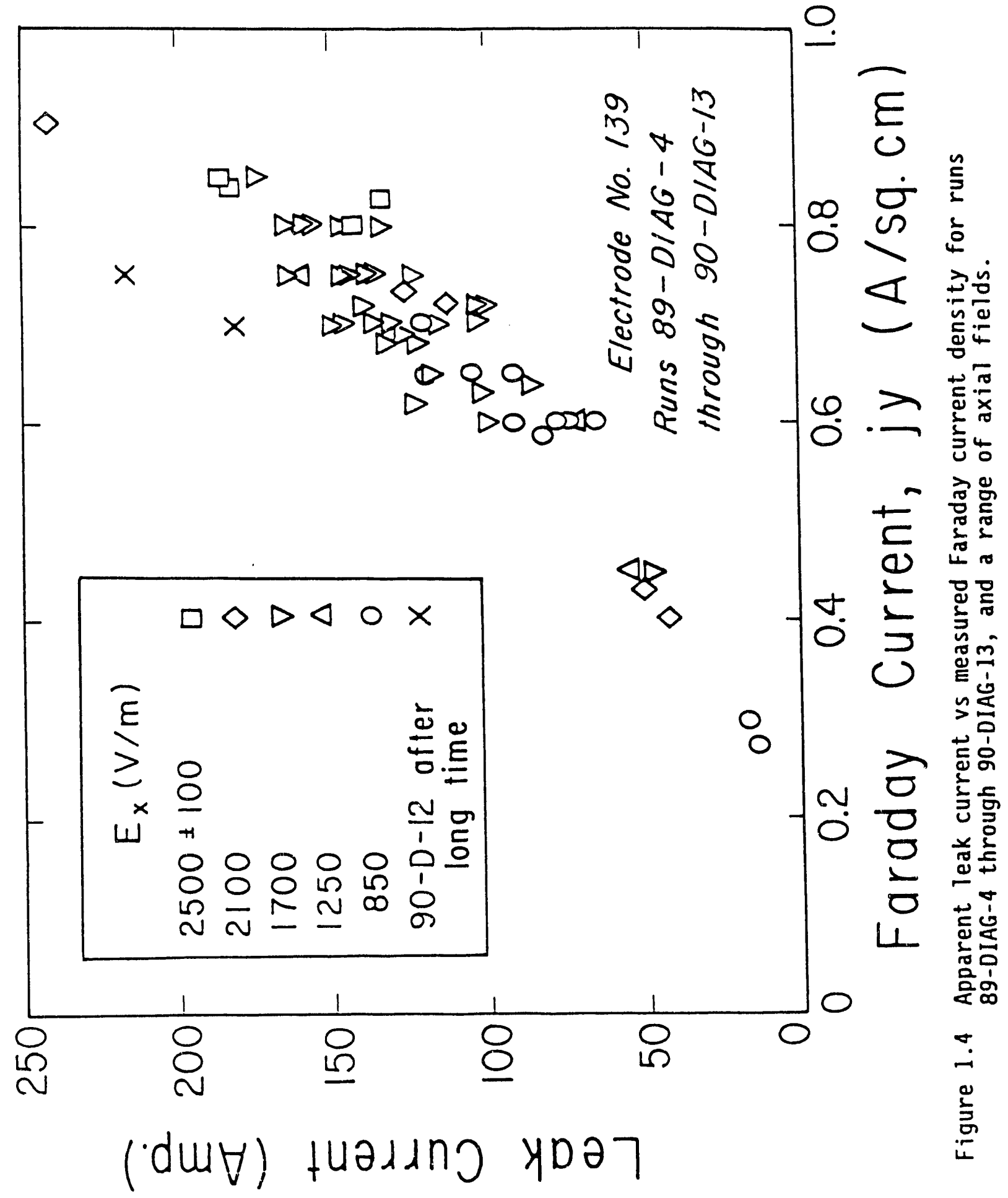




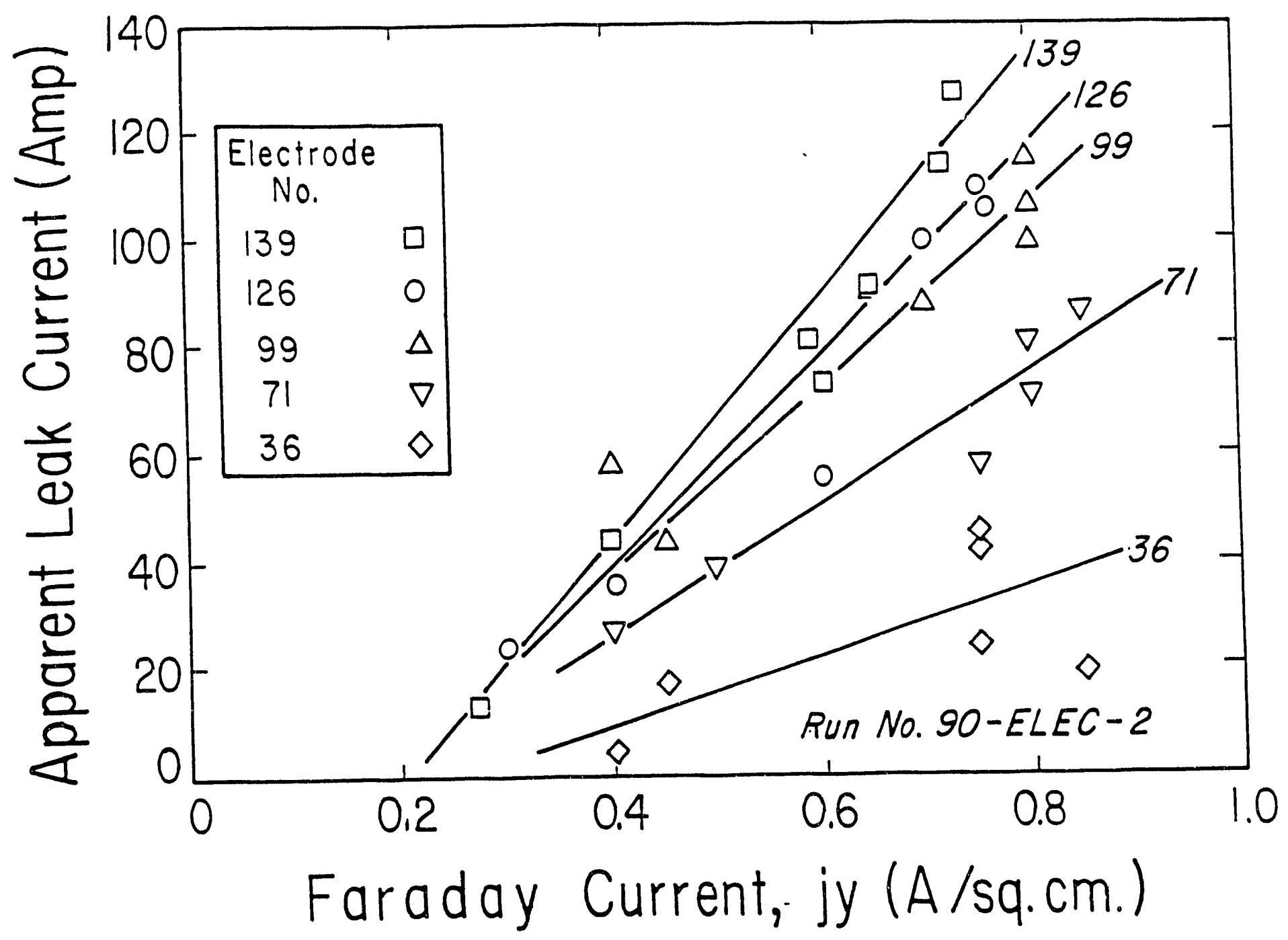

Figure 1.5 Leak current vs Faraday current, jy, at several axial locations. 


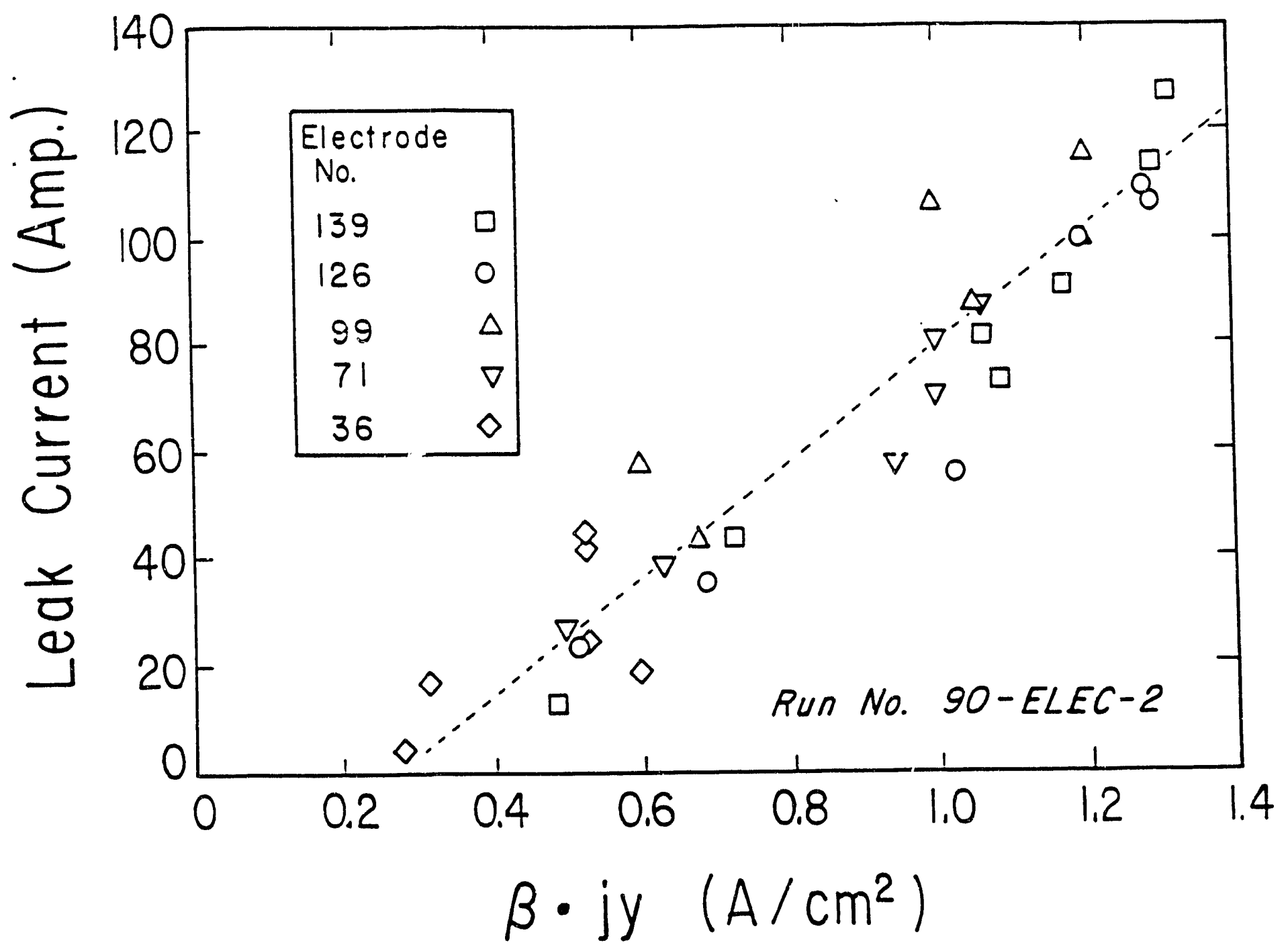

Figure 1.6 Leak current vs $(B * j y)$ at several axial locations. 


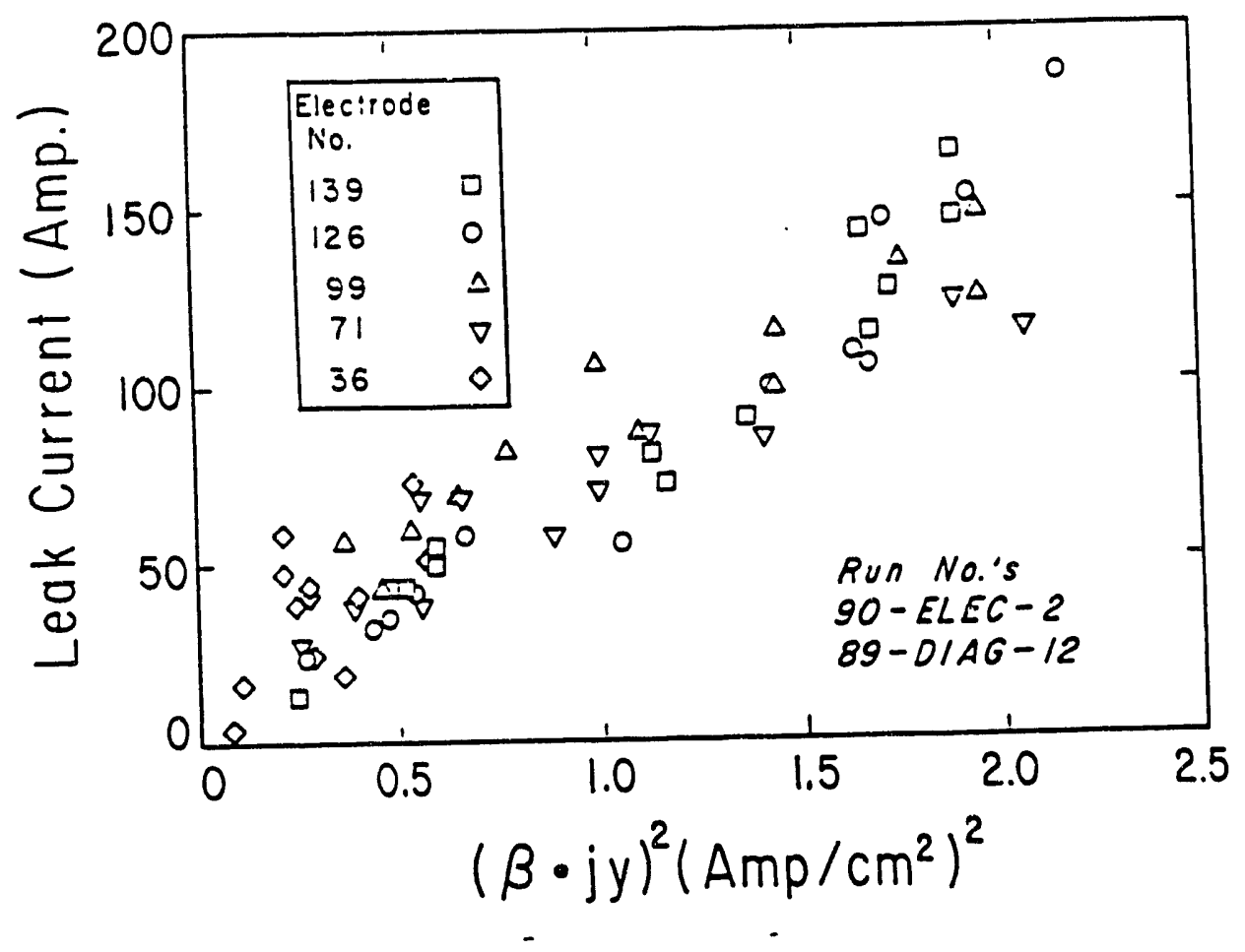

Figure 1.7 Leak current vs the square of $\left(B^{*} j y\right)$ at several axial locations. 
slag layer does seem to be significant. If we back off and consider only electrode locations near mid-channel, i.e. 126 and 139, the relative positions are considerably clearer, as is shown in Fig. 1.9. (Scatter tends to increase near the entrance. It becomes extreme at electrode 36 where all parameters are steep functions of axial location).

\section{Mechanisms}

The relative positions of the four sets of data suggest four possible apparent leak mechanisms. For convenience these will be called; resegmentation, single segmentation, normal slag leak, and whatever is left over, i.e. the "residual". Each will now be discussed.

\section{Resegmentation}

The difference between $\mathrm{Fe} O N$ and $\mathrm{Fe}$ OFF results may simply reflect an increase in effective electrode segment size. If, when $\mathrm{Fe}$ is off, the shorted intercathode gaps cluster on average in groups of size, Ns, then the current path between anode and cathode will be tilted as shown in Fig. 1.10 (For simplicity the current paths are sketched as if for Faraday loading). Consequently, the generator will behave as though there is an axial current density of approximate magnitude,

$$
\langle j x\rangle \approx(1+\alpha N s)(P t / Y) j y
$$

where $P t$ is the electrode pitch, $Y$ is the channel height, « signifies an average over $x$, and $\alpha$ is a number ranging from a maximum of unity down to a minimum of $1 / 2$ as current control effectiveness ranges from zero to 100 percent. It follows that, between $\mathrm{Fe}$ OFF and $\mathrm{Fe}$ ON runs, there will appear to be a difference in leakage current, $\delta I(l e a k)$, of approximate magnitude,

$$
\delta I(\text { leak) } \approx \alpha N s P t Z j y,
$$

where $Z$ is the channel width.

Assuming $\alpha=1 / 2$, Table 1.1 shows calculations of $\delta I$ (leak) for run $89-C F C$ 15, Cond. 7. The mean number of shorted gaps, Ns, was determined by averaging run data over 20 electrodes centered around the electrode in question. From Figure 1.9 it is evident that $\delta I$ (leak) has a lot of scatter. However it tends to lie in a range from roughly 20 to 70 Amp. Since the values in Table 1.1 Tie in this range it seems plausible that increased effective segment size indeed explains the difference between Fe OFF and ON.

Table 1.1

\begin{tabular}{clcc} 
Elec. No. & jy & Ns & SI(leak) \\
\hdashline 36 & -.9 & - & - \\
71 & .91 & 7 & 97 \\
99 & .88 & 4 & 54 \\
126 & .79 & 5 & 60 \\
139 & .7 & 5 & 63 \\
& .65 & 4 & 40
\end{tabular}




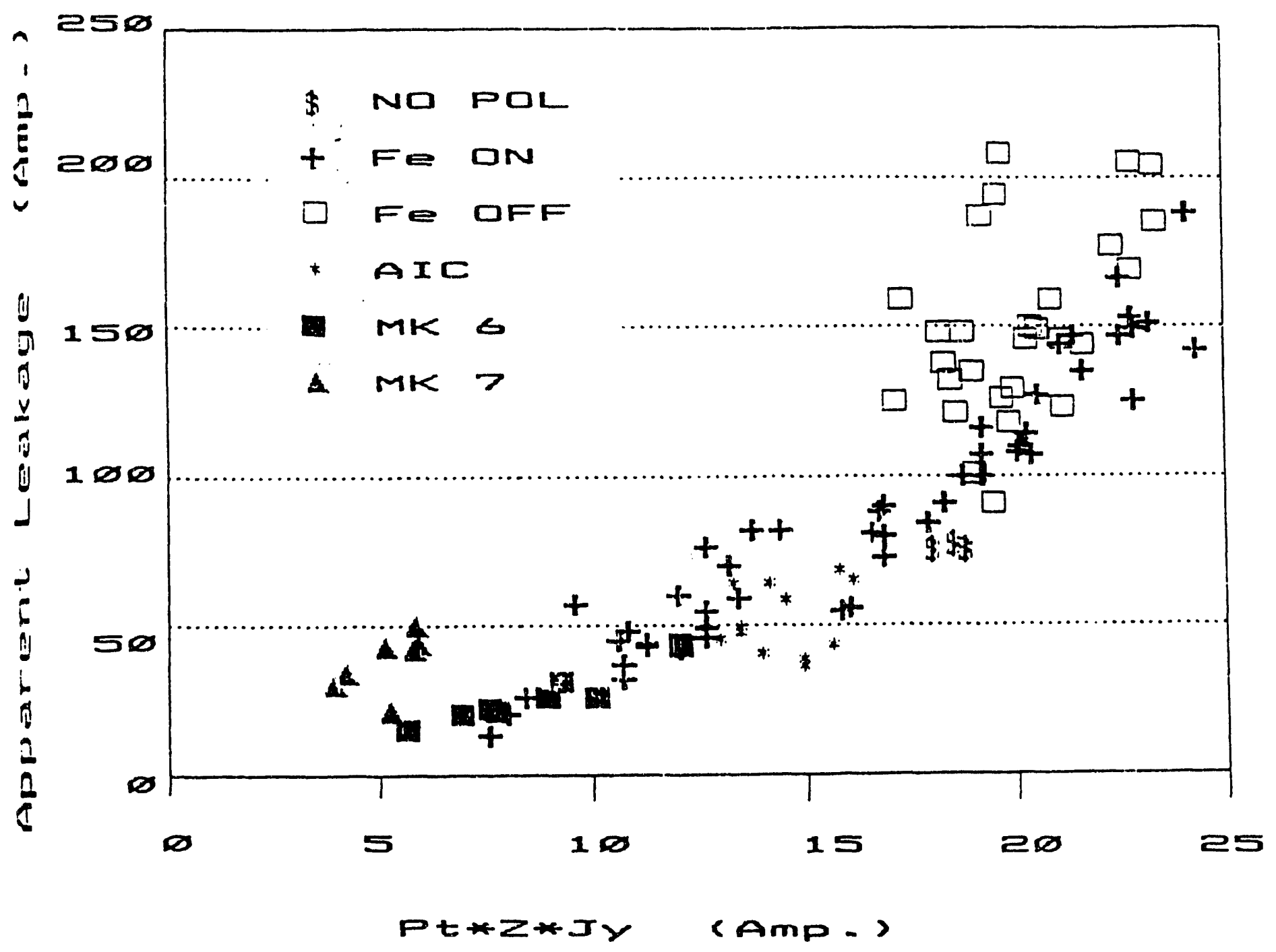

1.8 Apparent leak current vs electrode current for iron on, iron off, ash injected oil combustion (AIC), and a no iron-no polarization condition. Data from the Mark 6, Mark 7, and CDIF electrode locations $71,99,126$, and 139. 


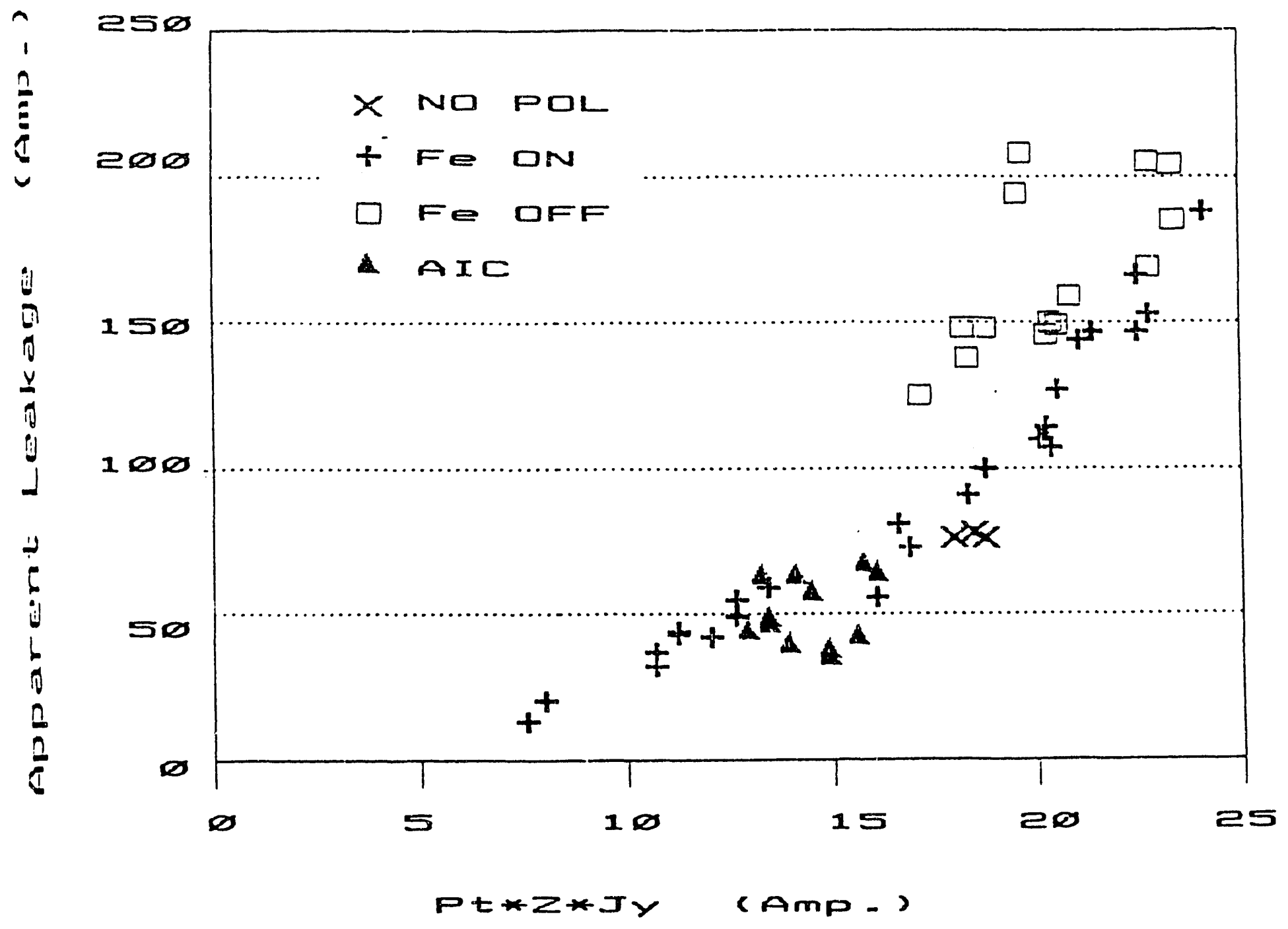

Figure 1.9 Same as figure 1.8 but with data from CDIF electrode locations 126 and 139 only. 


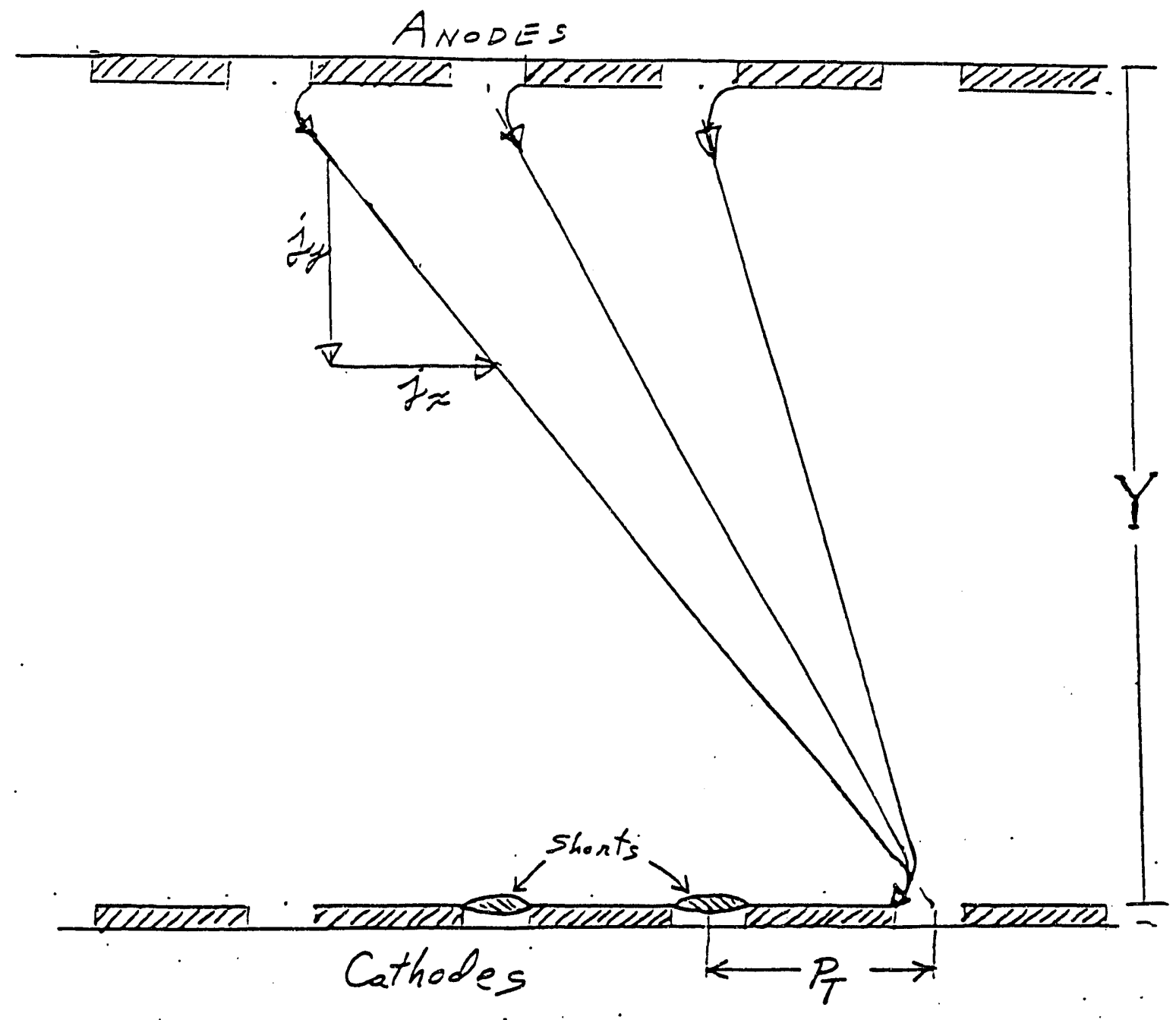

Figure 1.10 Finite segmenting effect magnified by shorted cathode gaps. 


\section{Single segmentation}

When $\mathrm{Fe}$ is $\mathrm{ON}$ we will still have an apparent leak due to the effect of a single segment. This can be estimated by setting Ns equal to zero in the equation for jx. A typical result for I(leak) in this case is around 20 amperes.

\section{Normal slag leak}

We know from the work of Pollina et al that the normal conductivity of slag as a function of temperature and composition (Ref.6) and we can therefore calculate an expected average conductivity of the slag layer for flow parallel to the surface.

Generally a value of about 10 ohms results for typical CDIF slag chemical composition and interface temperature. To calculate the axial current that results, let us use a typical axial electric field of 1700 volts $/ \mathrm{m}$. This value is especially interesting because it is not only typical but also the value that existed at electrodes 126 and 139 when the "No Polarization" data were taken.

With a slag layer thickness of $2 \mathrm{~mm}$ and a duct cross section at electrode 139 of $0.26 \mathrm{~m} \times .175 \mathrm{~m}$, the "normal" current works out to be 30 amperes, which is rather close to the difference between the No Pol. and $\mathrm{Fe} O \mathrm{ON}$ points in Figure 1.9. A reasonable inference is that the No Pol. data represents a slag layer that either did not exist or had a very high resistance. When the No Pol. data was taken, the burner had been on for only 20 minutes and seed had been on for only 90 seconds.

Table 1.2

1. Normal resistive leak along the slag.

2. Normal finite segmenting effect.

3. Resegmenting due to shorted gaps, if any.

4. Residual
$30 \mathrm{~A}$

$20 \mathrm{~A}$

$40 \mathrm{~A}$

$50 \mathrm{~A}$

TOTAL $140 \mathrm{~A}$

\section{The residual}

As shown in Table 1.2, we have now, at an electrode current of 18 amperes, accounted for about $40+20+30=90$ amperes of leakage current out of a total of $140 \mathrm{~A}$. The task now is to explain the remaining 50 amperes. This residual leakage current may be a real phenomenon, or it may be an artifact of the process by which the total leakage current was inferred.

A possible real phenomenon is to be found in a paper by Ishikawa, Nakamura, and Umoto, (Ref.7). These authors calculate that regions of high plasma temperature and high conductivity extend down-stream from the corner of each cathode as shown in Figure 1.11, which is taken from their paper. While values of apparent axial current are not given, the authors note that ".. the current sheet extends to the third cathode .." . They are speaking of a channel with an electrode pitch of $2.5 \mathrm{~cm}$. In the CDIF the same distance would extend to the fifth cathode.

How far must a current sheet (or arc column) extend in order to explain the 50 amperes? Such a current sheet should have much the same effect on the bulk of the plasma as a coarse segment does. Therefore the equations given above for the 


\section{Core}

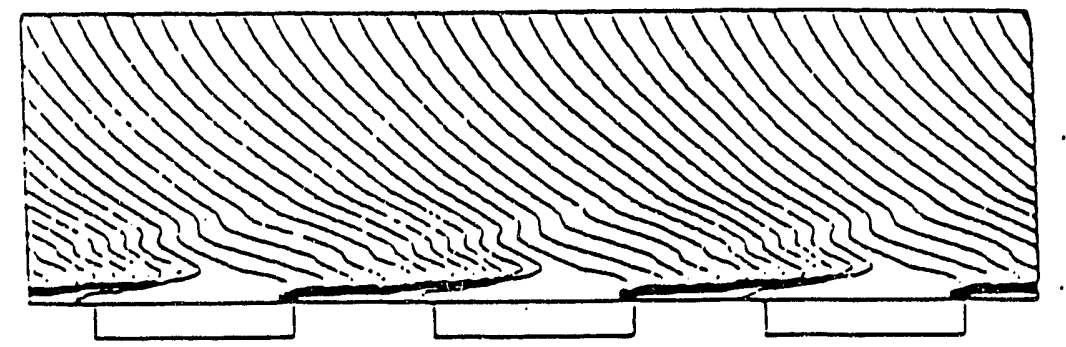

Cathodo

\section{Core}

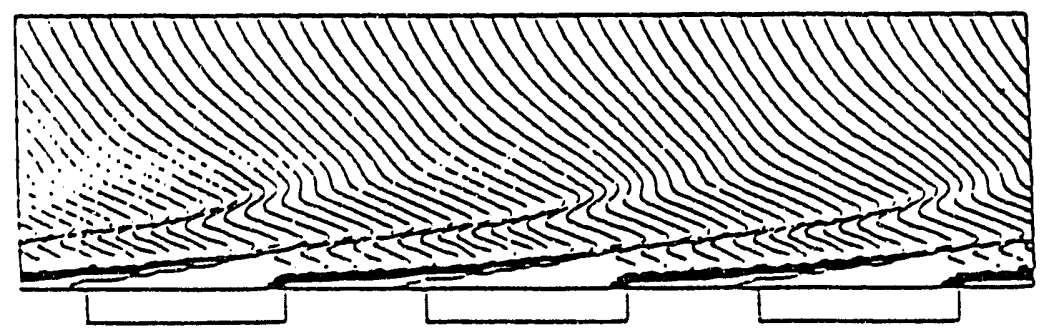

Cathodo

Figure 1.11 Finite segmenting effect magnified by plasma streamers. 
Fe OFF condition hold if we replace $(1+\alpha N s)$ by $\mathrm{Np}$, the effective length of the sheet expressed in terms of number of electrode pitches (The factor, $\alpha$, is effectively unity because each current sheet or arc column presumably attaches to a different cathode causing the current from each anode to cross the duct at the same angle. Note also that the "effective length" may be less than the actual length because the mean attachment point for the current is not at the end of the sheet). Therefore

$$
I(\text { leak,plasma }) \approx N p \text { Pt Z jy }
$$

which with typical numbers gives,

$$
I(\text { leak, plasma })=50 \approx N p(.0155)(0.175)(8000)
$$

Solving for $N p$ yields, $N p \approx 2.3$. In other words the sheet needs to extend a distance of a little over two electrode pitches. The calculations of Ishikawa et al suggest that this is possible.

Even though this phenomenon is not a leak through the slag but a thermal effect in the plasma, it may explain the typical appearance of the cathode slag after a run. This appearance is described by Farrar, et al. (Ref. 8). Typically, the cathode layer is noticeably thinner than it is on anode and side walls, especially immediately downstream of an open gap. Also the surface is often pockmarked, as by burst bubbles. Both facts suggest intense heating as might be expected from a current sheet (or arc column) pressed down upon it from above.

Now let us consider the alternative, that the apparent residual leakage current is an artifact of the method used for inferring it. There are similarities between all of the methods described in references 1 through 5 and thus all may be subject to similar uncertainty. However it is specifically the Faraday profile method described above and in references 4 and 5 that will be examined here. Possible sources of error are the following:

1) Assumed Hall parameter and average velocity values higher than the actual ones. These values are not based upon measurement but upon channel flow calculations. If they are too high by twenty to thirty percent the residual leak shrinks to about zero. This is unlikely because the deviation between calculated and measured pressure distributions seems to be significantly less than $20-30 \%$. This is a highly subjective judgement because the pressure data is unreliable, and typically exhibits tremendous scatter. However it is also true that in order to make the calculated static pressure rise by 20 to 30 percent some rather extreme assumptions must be made, for example that the effective roughness height of the slag surface is 1 centimeter.

2) Average conductivity higher than inferred. This value is inferred from the data by the method described above, and uses, among other things, the quantity, DV/DH obtained from the CDIF Preliminary Test Reports. It is assumed that, Eyc, the core value of the transverse electric field equals DV/DH. Thus the higher the value of $\mathrm{DV} / \mathrm{DH}$ (other things being equal) the higher the conductivity (more precisely, it's $z$ average) must be. However the value of DV/DH will be lower than Eyc if the side walls leak current in the transverse direction, and if the effective resistance between the plasma and each side-wall element is large. For side wall pegs of $x$ and $y$ pitch $p s$, the slag resistance, $r s$, and the resistance between peg and plasma, rp, are; 


$$
r p=\frac{\delta \star\langle\sigma c / \sigma\rangle}{p s^{2} \sigma c} \quad r s=\frac{p s}{\sigma s^{\star} t * p s}
$$

where $\delta$ is the boundary layer thickness, $t$ is the slag layer thickness, $\sigma s$ is the average conductivity of the slag layer, $\sigma c$ is the core conductivity of the plasma, and $\langle 1 / \sigma\rangle$ is the resistivity of the boundary layer. Typical values are, $p s=2 \mathrm{~cm} ., t=2 \mathrm{~mm}, \delta=1 \mathrm{~cm}, \sigma c=5 \mathrm{~s} / \mathrm{m}, \sigma s=10 \mathrm{~s} / \mathrm{m}$, and $\langle\sigma c / \sigma\rangle=2$. Then as a result,

$$
r p / r s=0.8
$$

performing a circuit calculation with this value predicts about a $20 \%$ difference between DV/DH and Eyc. This is not quite enough.

In order to explain away the residual leak, the measured DV/DH must be low by about $40 \%$. To be this low requires greater than expected transverse slag conductivity $(-50 \mathrm{~S} / \mathrm{m})$, and/or resistivity between plasma and slag surface ( 2 $\mathrm{m} / \mathrm{S})$. While both numbers are higher than expected, neither one can be entirely ruled out at this point.

3. Transverse leakage causing the actual core transverse current to be higher than that actually measured. This causes actual leakage to be greater instead of less than apparent leakage. In any case, for any plausible slag conductivity, the effect is small.

4. Surface voltage loss. This refers to a voltage loss anywhere else but in the region of uniform jy within the plasma. For example arc drop or drop across the slag layer. These must certainly exist. If, however, reasonable estimates of this voltage are included in the data analysis the apparent residual leak is still substantial and the inferred uniformity factor, $\langle\sigma\rangle\langle 1 / \sigma\rangle$, becomes unreasonably close to unity.

In summary, none of these sources of error, acting alone, seem likely to be of sufficient magnitude to explain away the need for a fourth, residual, leakage mechanism. However, if effects number 2 and 4 are both present, i.e. if both a distorted transverse voltage profile and a surface voltage loss occur together, then the required values, while still high, are more plausible, thus leaving all of the apparent leak explained by known and understood phenomena. However, before accepting this explanation, we must consider the results obtained from two other methods of data analysis, which will be discussed in the following two sections. 


\subsection{LOAD SWEEP; METHOD 2}

This method of data analysis makes use of the data obtained during a load sweep. Therefore it is restricted to those runs during which a load sweep is carried out. It produces results for the entire length of the channel, an advantage over the Faraday transverse profile method which is restricted to 5 locations in the front half of the duct. It does not directly produce estimates of apparent leakage current but does produce estimates of the Hall parameter which turns out to be equally useful, as will be seen. Additionally, it produces estimates of some of the same parameters that the profile method does but based upon different experimental data, thus providing a useful opportunity to check for consistency.

However velocity estimates are still required including estimates of it's rate of change with load. Also required for greatest accuracy is the rate of change of the Hall parameter with load. The most important requirement, and one that is not always met, is constant burner conditions during the load sweep. It takes only a small change in flame temperature to cause a large change in electrical conductivity and in all the data that depend upon conductivity.

This method was first developed by $W$. Daniel, and used by him to analyze a number of CDIF runs (Ref. $9-12$.). A variation of his method has been employed on this project, and is described below.

First, average transverse current density, $\langle j y\rangle$, and load current density, $\langle j L\rangle$, are plotted versus axial field, Ex, for every tenth electrode location, i.e. for about 20 locations. Figures 2.1 and 2.2 show examples of plots for five mid-channel locations. Here, $\langle j y\rangle \equiv \mathrm{Ie} /(p t \star Z)$ and $\langle j L\rangle \equiv \mathrm{IL} /(Y \star Z)$, where Ie is the measured current to an electrode, IL is the diagonal load current, $p t$ is the electrode pitch, while $Y$ and $Z$ are the local duct dimensions perpendicular and parallel respectively to the magnetic field vector.

A line is drawn through the points and extrapolated to obtain the local short circuit values of $j y$ and $j L$ and the local open circuit values $(j L=0)$ of jy and Ex. Daniel accomplishes essentially the same thing mathematically without needing to find the open and short circuit values. However, finding these values is useful for understanding qualitatively the effect on performance that each parameter has.

These values are then put into the right hand side of the following equations in order to infer the value of the parameters on the left hand side.

$$
\begin{aligned}
& B_{s c}=j L_{s c} / j y_{s c}-\Phi \\
& V e s / Y=u_{o c} B-\left(B_{o c}-\Phi\right) * E x_{o c} /\left(j y_{o d} / j y_{s c}-1\right) \\
& \langle 1 / \sigma\rangle_{s c}=\frac{\left(u_{o c} B-V e s / Y\right) / j y_{s c}}{1+B_{s c}^{2}} \\
& \langle\sigma\rangle_{o c}+\sigma s=j y_{o c} *\left(B_{o c}+\Phi\right) / E x_{o c} \\
& \langle\sigma\rangle=r s /\langle 1 / \sigma\rangle
\end{aligned}
$$




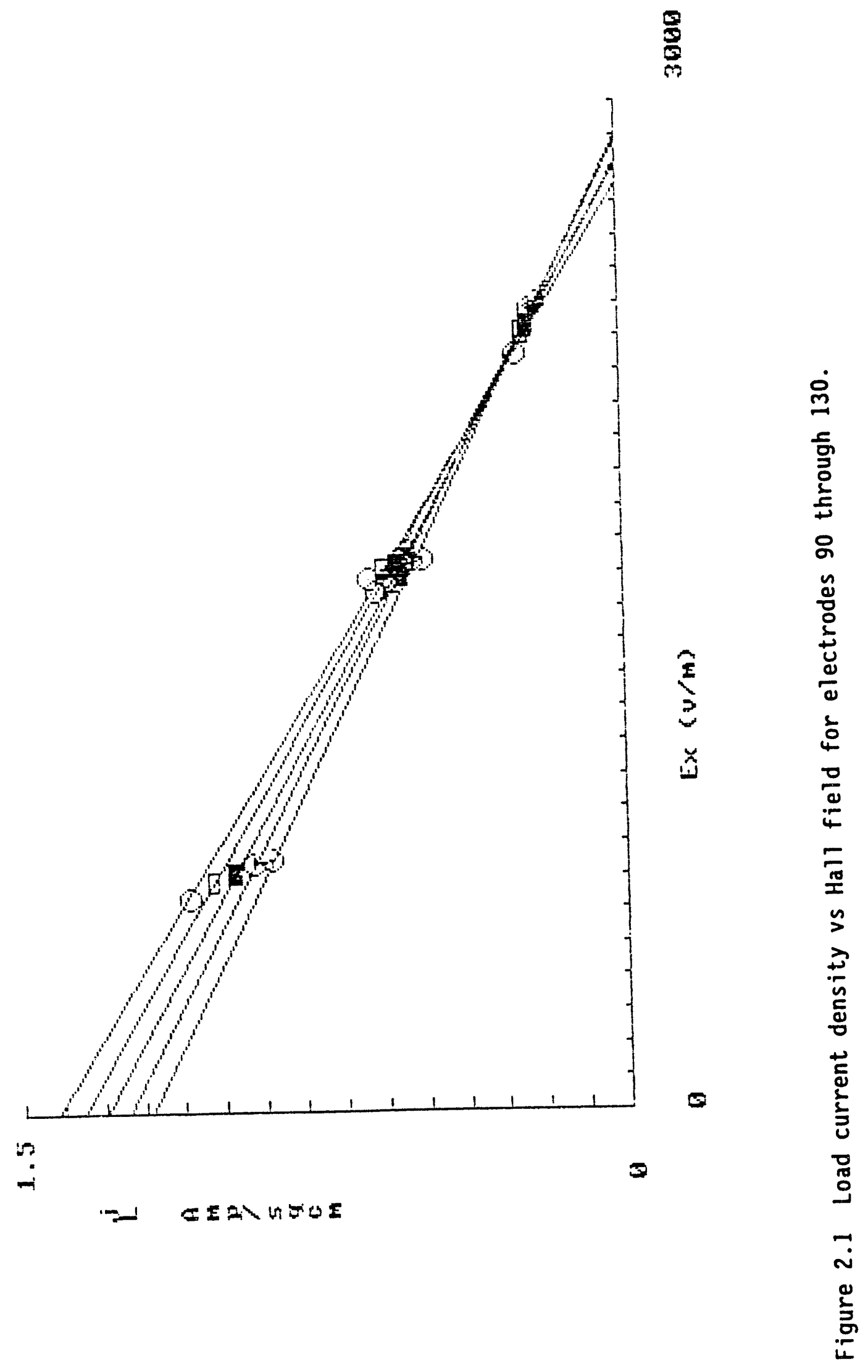




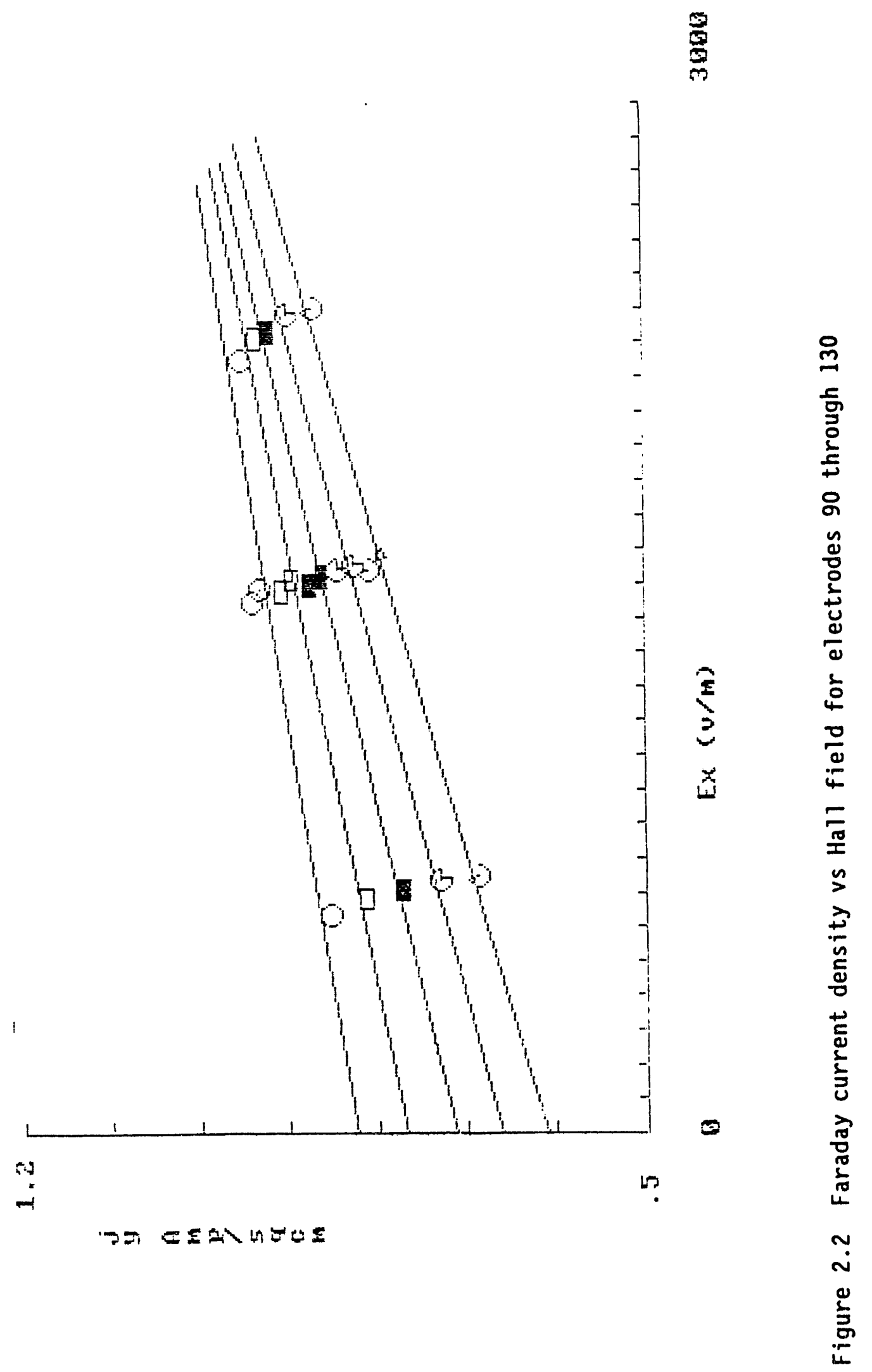


The subscripts, $s c$ and $o c$ denote short circuit and open circuit values respectively. In equation $2.5, \mathrm{rs}$ is an estimate of the value of the product, $\langle\sigma\rangle^{*}\langle 1 / \sigma\rangle$. This product is believed to have a value in the range $1.1 \pm 0.1$, under all conditions likely to be encountered. Thus equation 2.5 should yield an estimate of average conductivity accurate to within $\pm 10 \%$. The value if $r s$ is based upon experimental data and boundary layer theory.

Equations 2.1 through 2.4 are derived from the general $\mathrm{Ohm}^{\prime} \mathrm{s} 1 \mathrm{law}$ including the Hall effect, and the equation for diagonal load current (See equations 1.1 through 1.5 in section 3.1). The equations actually used in the data analysis program are somewhat more complicated because they include terms for transverse leakage. They can be found in Appendix A. A simplified version of the equations has been presented above in order to better show the physics.

Note that equation 4 gives only the sum of plasma plus slag conductivity. It seems that there is no way to get anything else just from the channel performance data that is usually recorded. To determine each individually more data is needed, such as a direct measurement of $\sigma$ s. Attempts to do this are reported in reference 1.

Alternatively an additional assumption can be made, namely the value of the product $r s, i . e .\langle\sigma\rangle\langle 1 / \sigma\rangle$. As noted above, numerous studies suggest that this product is always greater than one but less than about 1.2 . Therefore if we assume a "reasonable" value, for instance 1.1, and use it to get an estimate of $\langle\sigma\rangle$ from Equation. 5 we should have the true value to within perhaps $\pm 10 \%$.

Note that equations 2.2 and 2.4 require an estimate of the ratio of open to short circuit $B$ since only the short circuit value is "measured" by Equation 2.1 , and Equation. 2.5 requires an estimate of the ratio open to short circuit resistivity, $\langle 1 / \sigma\rangle$. Note also that Equations 2.2 and 2.3 require estimates of mean velocity at both open and short circuit. For these we are largely dependent upon values obtained from computer calculations of the channel flow. The accuracy of these should be sufficient, but experimental verification in the form of good static pressure measurements would be valuable. Al so valuable, but more costly, would be dynamic pressure measurements or direct velocity measurement by laser techniques.

Figures 2.3 through 2.6 show the results of analyzing run 90 Diag 3 by this method using time-averaged data furnished by Daniel. Figure 2.3 shows the inverse of the transverse resistivity, which is similar to but generally about 10 percent lower than the average plasma conductivity, as discussed above. It al so shows the apparent average Hall parameter, and the plasma nonuniformity factor, $G$.

Figure 2.4 shows the surface electrode drop and the slag conductivity. These results exhibit considerable noise because to get them requires finding small differences between large numbers. Figure 2.5 shows a least-squares fit to the data. The numerical values and the trends with axial distance are about as expected. In particular, the value of the slag conductivity of from 5 to 10 mho/m, is right in the range expected for "normal" behavior based upon known values of slag conductivity vs temperature. It shows no sign of the abnormal values required to explain high leakage current.

The only significant abnormality in the results of this analysis is the axial distribution of the Hall parameter, $B$, shown in figure 2.3. This can be better seen in figure 2.6. Here values of apparent $B$ obtained by Daniel from five different runs, are shown together with the values expected on the basis of channel modeling calculations. Modeling results are shown for the highest and lowest load currents usually employed in a load sweep. The apparent Hall parameter falls reasonably well into the expected range in the last half of the 
duct but is markedly lower in the first half, for all runs.

This is a significant result. The question now is which is correct, the values inferred by this method from experiment, or the modeling results? As we will see, both in a sense seem correct and, moreover, tie in with results from the other methods of data analysis. In any case, since $B$ is a function of pressure, it underscores the need for and value of good pressure measurements.

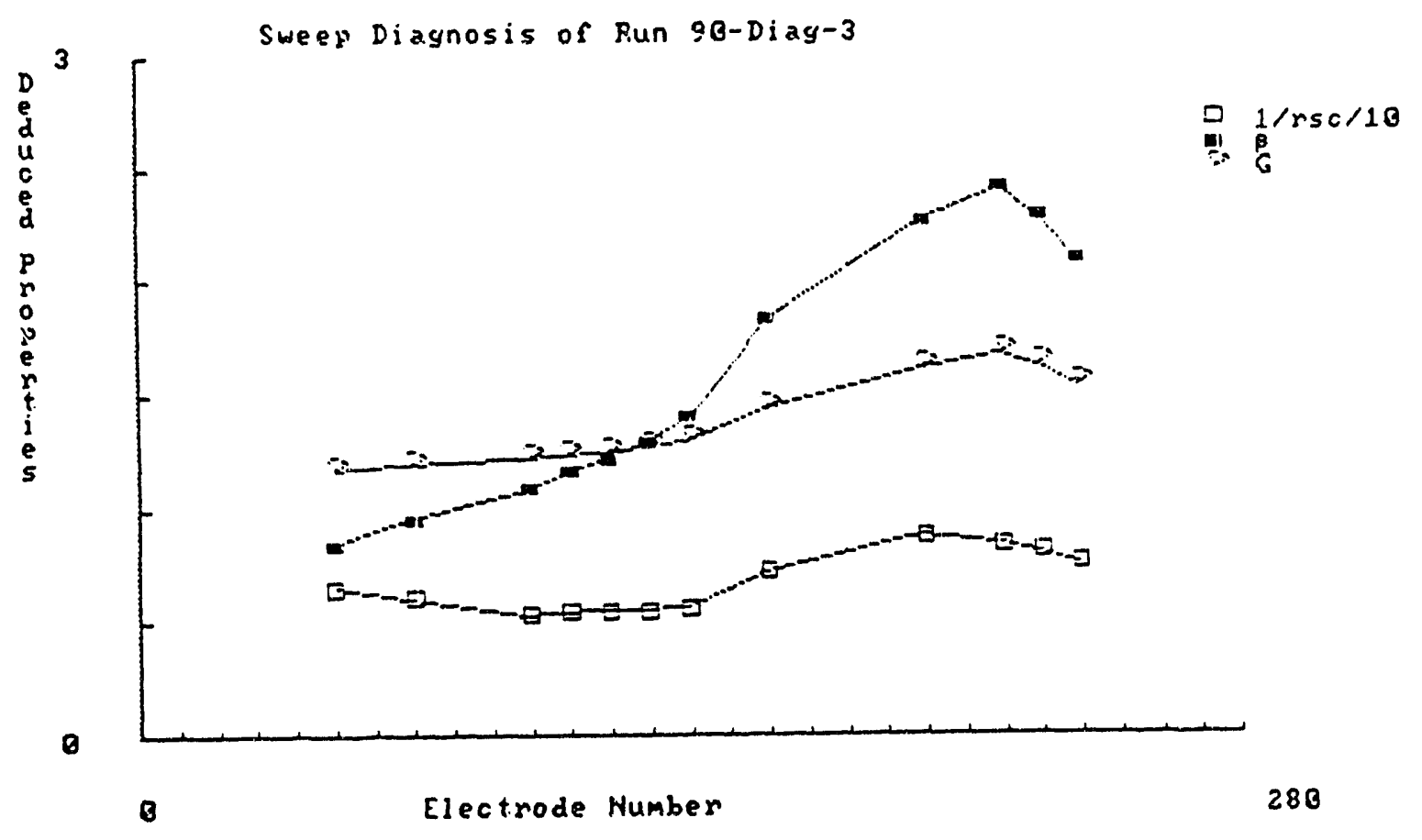

Figure 2.3 Sweep diagnosis of run 90-DIAG-3 for resistivity, rsc, Hall parameter, $\beta$, and nonuniformity factor, $G$. 


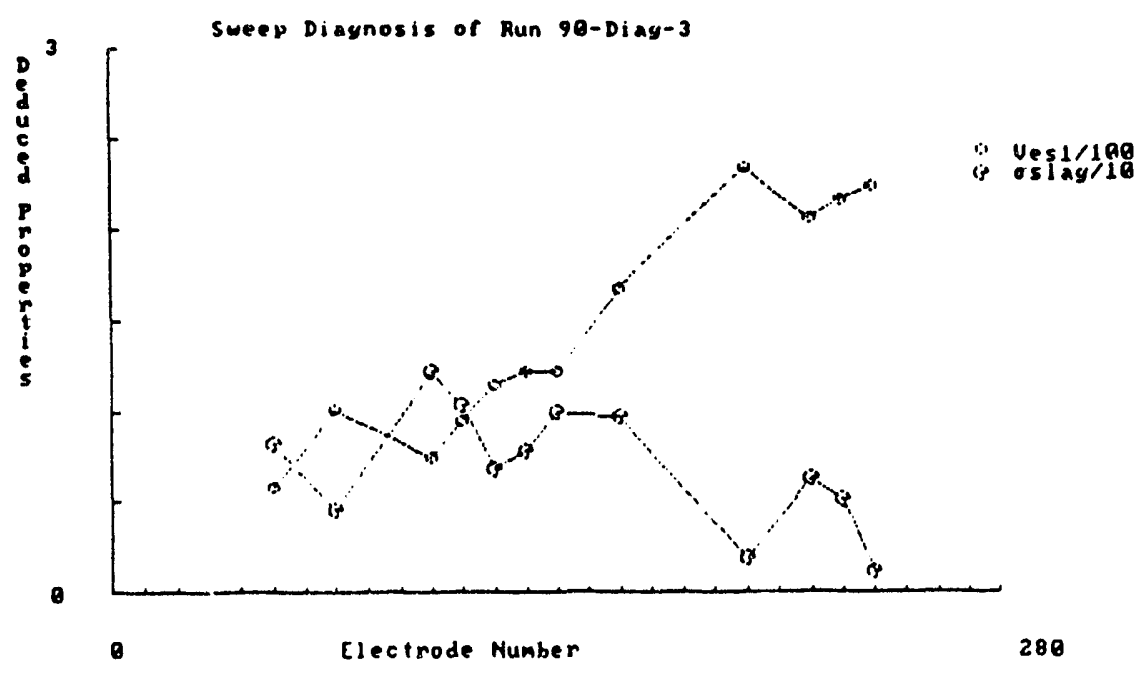

Figure 2.4 Sweep diagnosis of run 90-DIAG-3 for surface voltage drop, Vesl, and slag conductivity, $\sigma$ slag.

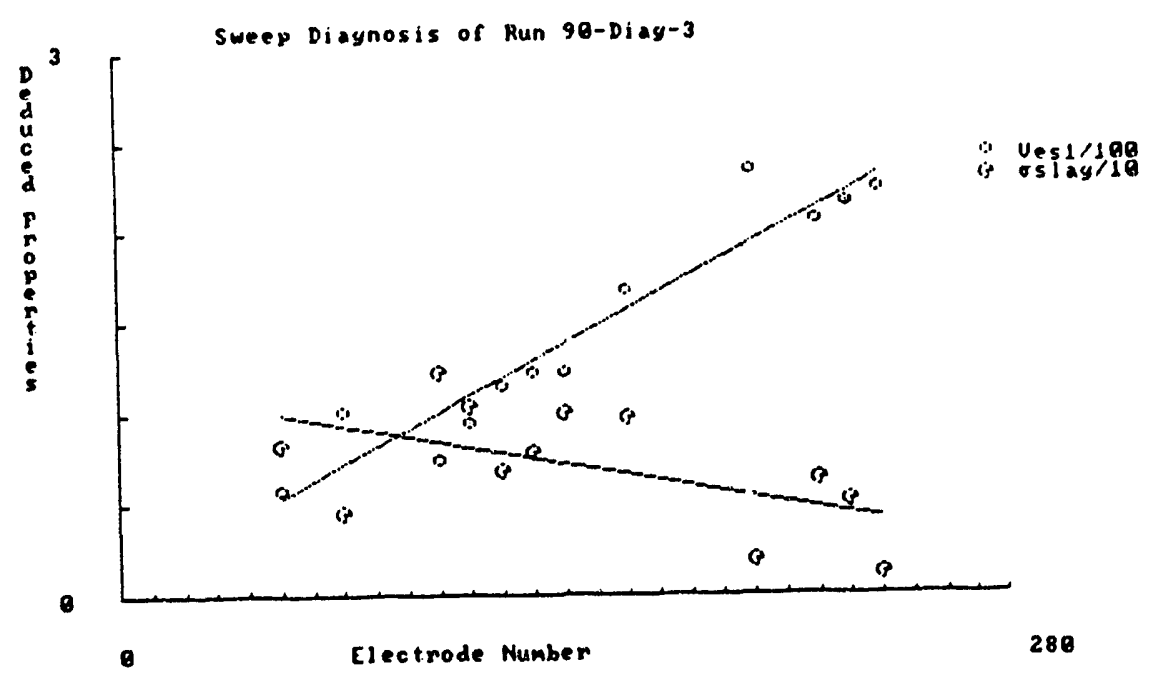

Figure 2.5 Least squares fit to the data in figure 2.4.

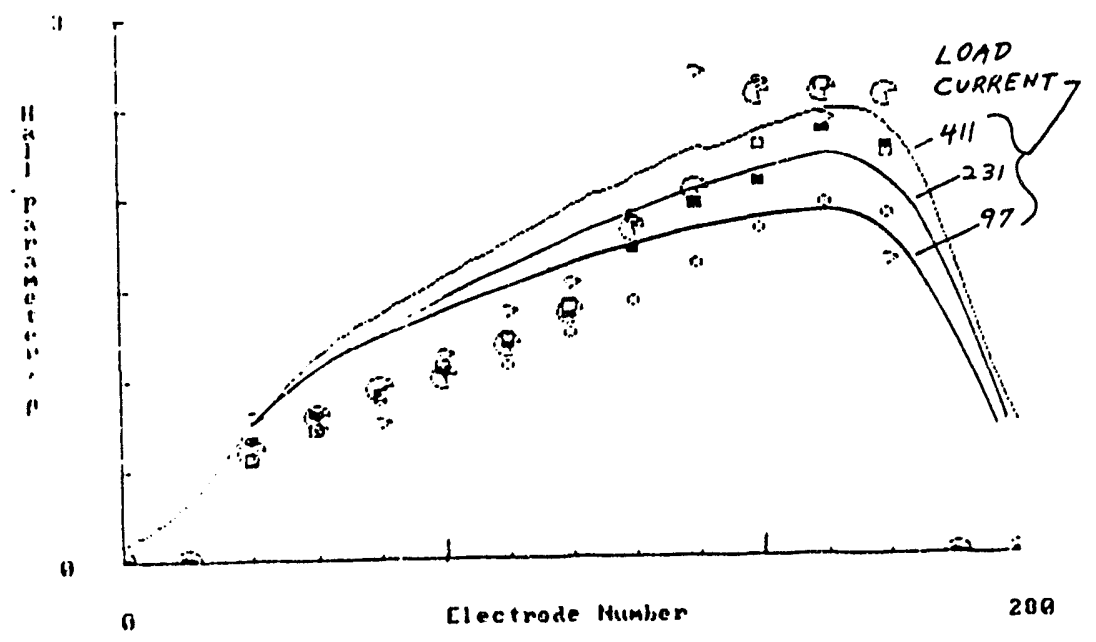

Figure 2.6 Hall parameter vs axial position. 


\subsection{METHOD THREE, THE MODELING METHOD.}

The modeling method consists of calculating the performance, gas-dynamic and electrodynamic, of an MHD generator and comparing it to the measured performance. Global measures of performance, i.e. the net power output, the output current, and the output voltage are compared. Also compared are more detailed measures such as; the split between power to the diagonally connected load and power to the link resistors (if any), and the axial distribution of several quantities, namely the transverse current, the transverse and axial electric fields, the no-load conductivity (if measured), and the pressure.

Built into the model are a number of equations with adjustable parameters which model the effects of axial and transverse current leakage, plasma nonuniformity, incomplete combustion, boundary layer impedance, surface voltage loss, wall roughness, or any other process which is suspected of affecting global performance and/or axial distributions. In principle, if all these parameters are adjusted until the model and the experimental results agree in all respects for all runs of the generator and for all load conditions, then an accurate picture of the effect of each process is obtained.

In practice this is difficult to accomplish. There are too many parameters, the effects of which often overlap. Al so there may be reasons for suspecting that some parameters, for example burner output temperature, vary significantly from one run to another and/or from one load condition to another, but no good basis for quantifying the variation. This increases still further the number of combinations of values that need to tested.

Never-the-less, when used in combination with the other methods, the modeling method yields valuable insights, and indeed may be considered the ultimate test of any proposed leakage or other loss mechanism. The results also help in determining what additional measurements are most feasible and would most increase understanding. following:

In the course of this study the parameters varied included principally the

Axial leakage resistance, magnitude and distribution.

Transverse leakage resistance.

Plasma nonuniformity.

Surface voltage drop.

Surface roughness.

Duct entrance enthalpy.

Virtual flow constriction as might be caused by separation.

Reduced Hall parameter, to simulate molecular collision cross-sections larger than the literature reports.

A parameter linking leakage to Faraday current.

A typical set of curves comparing modeling calculations with data for runs number 90-DIAG-3 are shown in Figures 3.1 through 3.5. Significantly, the best agreement was obtained by including in the modeling program a parameter linking leakage current, Ik, to Faraday current, jy, plus leakage proportional to axial Hall field, Ex, by means of the following equation.

$$
I K=A *\left(\sigma_{x} \star E_{x}+C_{j} * j_{y}\right)
$$




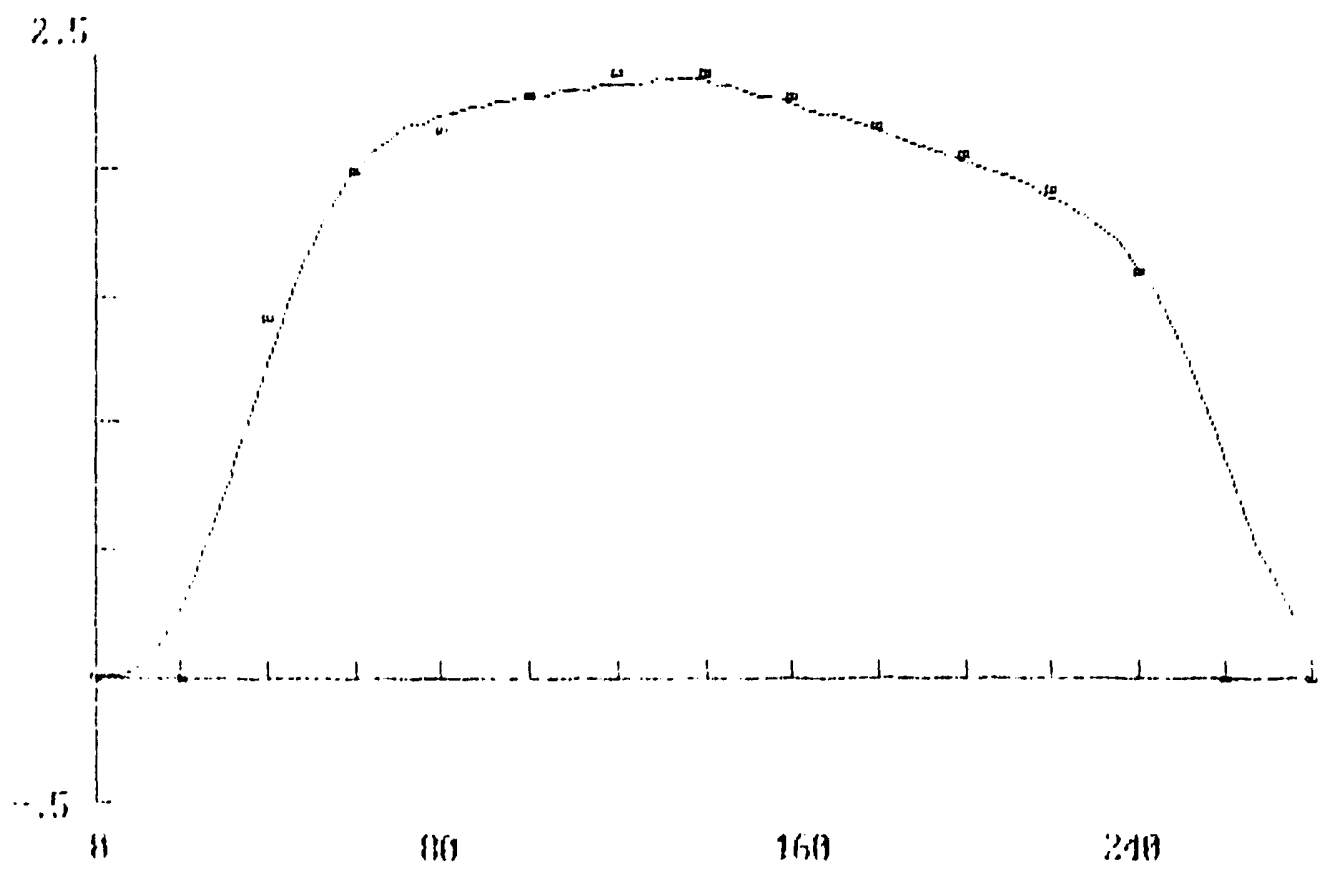

3.1 Hall field vs axial position for a load current of 231 Amp.

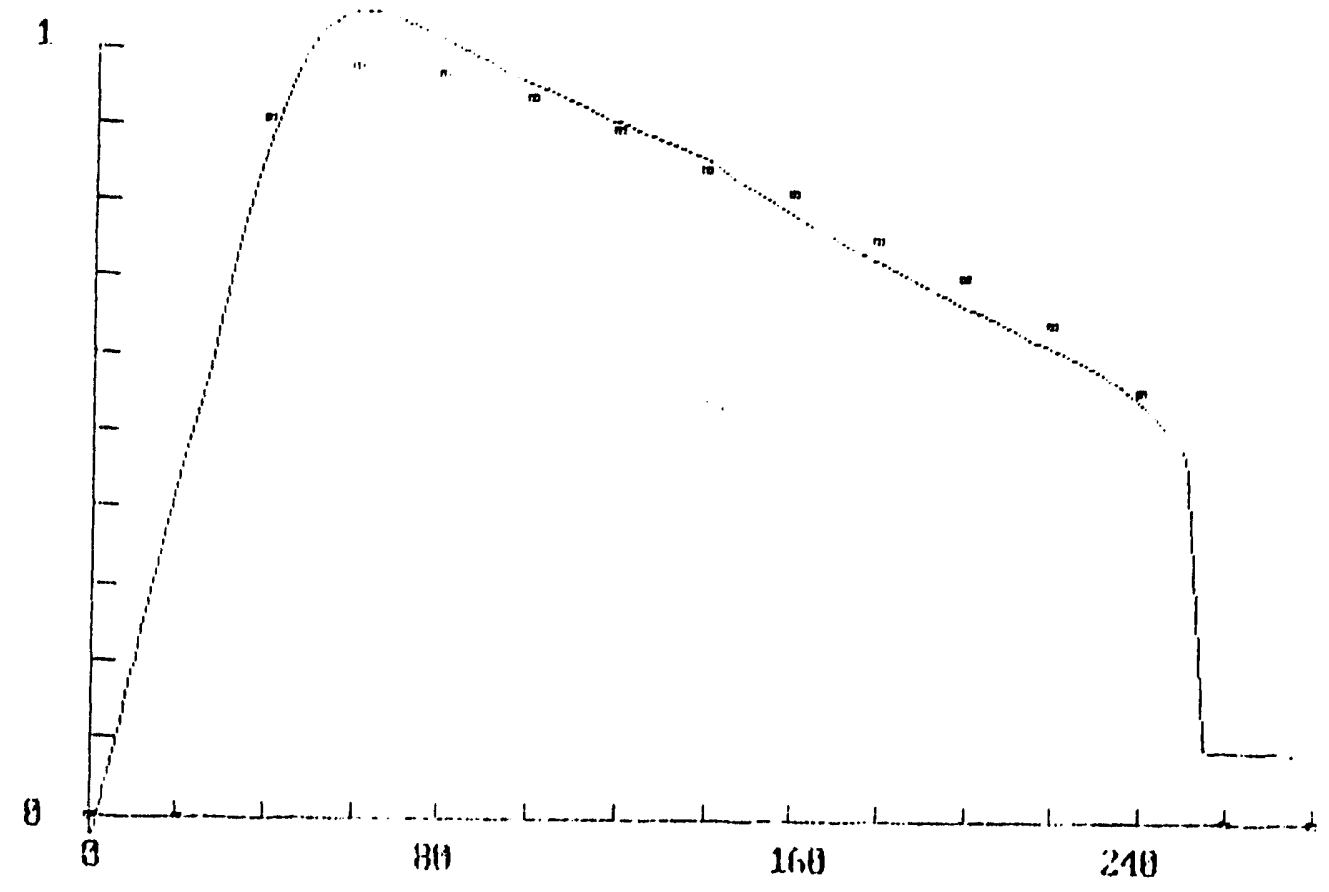

3.2 Faraday current density vs axial position for a load current of 231 Amp. 


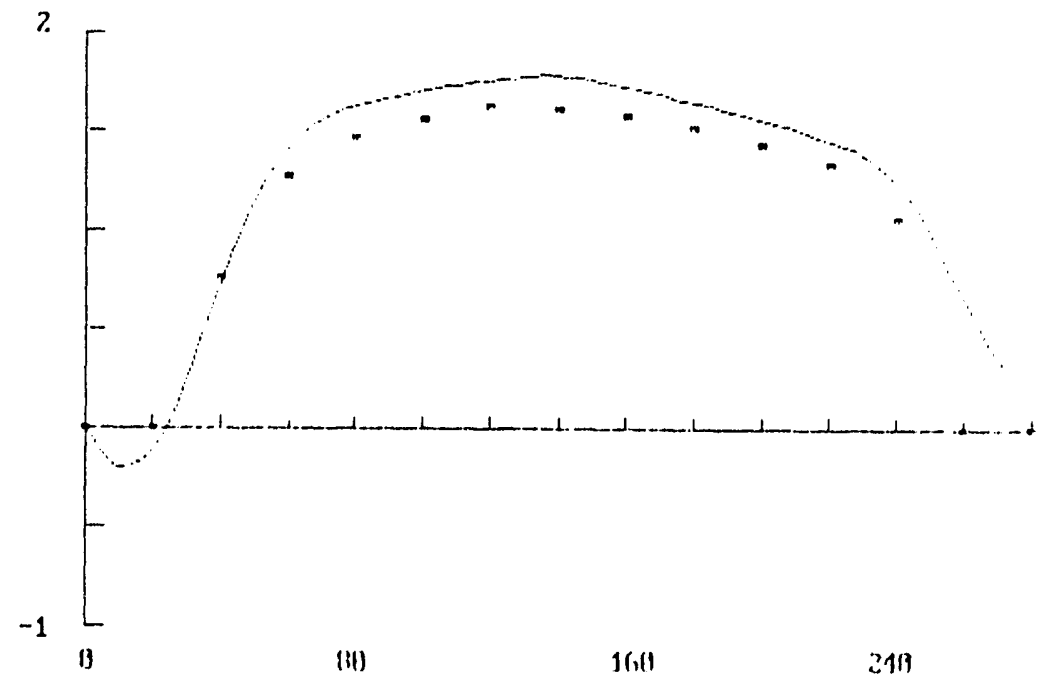

3.3 Hall field distribution for a load current of 414 Amp.

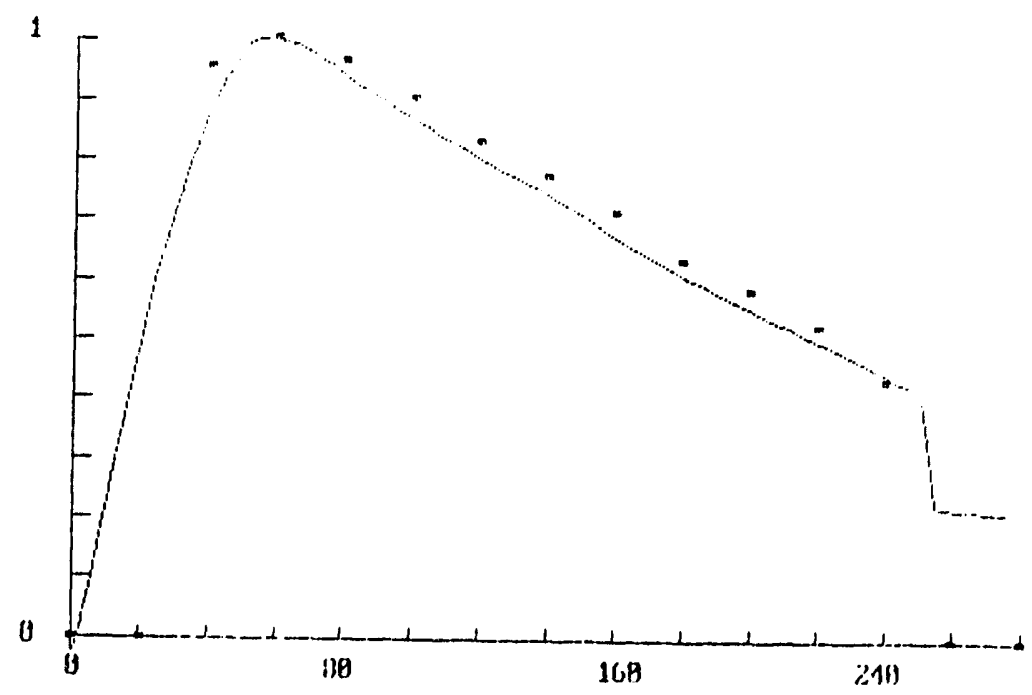

3.4 Faraday current distribution for a load current of 414 Amp.

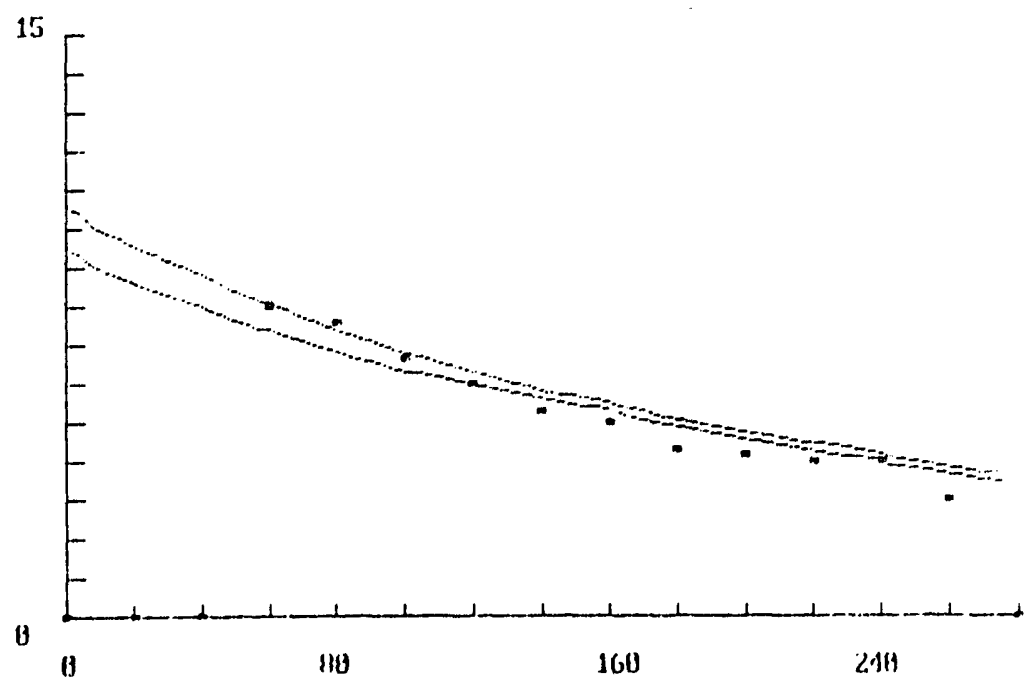

Figure 3.5 Unloaded electrical conductivity distribution. 
where $A$ is the duct cross-section, $\sigma_{x}$ is the axial leakage conductivity, defined by,

$$
\left.\sigma_{x} * A=\langle\sigma(s) a g)\right\rangle * P * t
$$

where $P$ is the duct perimeter, $t$ is the slag layer thickness, and $\langle\sigma(s) a g)\rangle$ is the conductivity of the slag averaged over it's thickness, $t$, using curves of conductivity versus temperature measured by Pollina et al (Ref. 6). The term containing Ex is the leakage normally expected based upon known properties of slag, and is relatively small in magnitude, generally about 25 amperes in the CDIF.

Finally $C_{i}$ is a number which links Ik to the transverse current density, $j_{y}$. While assumed to be a constant for most of the Method 3 calculations, there is evidence and physical arguments suggesting that there is and ought to be a functional dependence upon axial distance, $x$.

The most noteworthy result of the many modeling calculations that have been made is that $C_{j}$ is the only adjustable parameter that one can set and forget, which is to say, it is the only parameter for which a single value can be found that works well for all loads and almost all runs. Only when iron oxide slurry injection is turned on and off is a change necessary, and this is to be expected. In contrast, if agreement with experiment is sought by adjusting any other parameter in the list given above, a different value tends to be required for each stoichiometry, $\mathrm{N} / \mathrm{O}$ ratio, seed rate, and electrical load condition. Most notably, it requires that $\sigma_{x}$ be assumed to vary with load.

The physical significance of this result and it's relation to the results of Methods 1 and 2 will be discussed in the next section.

\subsection{CONCLUSIONS}

All three of the methods of analysis have different limitations. Because they differ, they can be compensated for by combining the results of all methods. The principal limitations and conclusions from all three are listed below.

A. The data upon which method one is based may be affected significantly by transverse leakage current, the magnitude of which is unknown. Transverse leakage does not seriously affect the accuracy of method two.

B. Method number one, the transverse profile method, suggests;

a. There is an apparent axial leakage current proportional, not to axial voltage gradient, $E_{x}$, but to transverse current, $j_{y}$, times duct width, $z$.

C. Method number two, the load-sweep method, suggests the following:

a. The slag layer conductivity, $\sigma_{x}$, is about as expected from direct laboratory measurements of slag conductivity vs temperature and composition. volts.

b. Electrode surface voltage drop is about as expected, i.e. about 100

c. The Hall parameter, $B$, is not as expected. It is too low, in the first half of the duct, when compared with the value predicted by the modeling method or by calculations based upon measured values of magnetic field strength and static pressure. 
D. Method number three, the modeling method, suggests;

a. There is an apparent axial leakage current proportional, not to axial voltage gradient, $E_{x}$, but to transverse current, $j_{y}$, times duct area, $Y * Z$.

E. Since $Y$ is almost constant in the CDIF, Methods one and three are saying the same thing. It will be shown in the following discussion that Method two is saying the same thing also.

Discussion as

Equation 1, of method two, which is used to determine $B$, may be rewritten

$$
I(\text { load })=A *(B+\Phi) * j_{y} \quad 4.1 . b
$$

This equation in effect assumes there is no axial leakage current. But if there is some process causing an axial leakage proportional to $j_{y} * Z$ or $j_{y} * Y * Z$, then equation 1.6 should be modified to read,

$$
I(\text { load })=A *\left(B+\Phi-C_{j}\right) * j_{y} \quad \text { 4.1.C }
$$

where $C_{j}$ is a constant (a)though it may change with axial location or iron and seed feed rate, or other operating conditions). Note that $C_{j} c a n$ be interpreted as a reduction in either effective $B$ or effective connection angle, $\Phi$. In either case it seems that the results of all three methods say, or imply, essentially the same thing, or rather one of two things. Either

1. The actual $B$ is lower than expected.

2. There is an apparent leak proportional to jy.

In order to account for low $B$ either the static pressure must be higher than modeling and experimental data implies, or electron-molecule collision cross-sections must be larger than everyone thinks they are. Since neither seems likely (although not impossible), it seems more likely that there is a substantial leak, and it is largely proportional to jy. This is a surprising result since one customarily expects a current to be driven by a voltage. If it is not then we must attempt to explain why in order to understand what is going on in this critical region of the generator.

one straightforward example of an apparent leak proportional to $\left(j_{y} * Z\right)$ is that due to finite electrode size. The effective electrode size is increased by interelectrode shorts, as was discussed under Method one.

Moreover, any nonlinearity in the conductivity of the plasma or the slag layer may tend to make leakage seem proportional to jy rather than Ex. For example, in a plasma, an electric arc has a resistance so nonlinear that under a wide range of current values it exhibits approximately the same voltage. In this case current is determined primarily by the capacity of the current source (in our case the MHD generator), with virtually no apparent dependence at all upon the voltage (Voltage may then be thought of as an effect of current flow, not it's cause).

Also discussed under Method One was the possibility that effective size is increased by streamers extending downstream from the trailing edge of cathodes. These might be in the plasma, as described by Ishikawa (Ref. 7) and illustrated 
in Figure 1.11, or they might lie on the surface of the slag layer. A streamer of plasma or of hot, highly conducting slag on the top surface of the slag, are separated from the metal electrodes by cooler slag having a relatively low conductivity. Thus measurements of interelectrode voltage would not necessarily reveal shorts since these measure voltage at the bottom of the slag layer. This assumes that the transverse position of streamers from neighboring cathodes is displaced enough so that the streamers do not intersect, at least on average.

Another possibility is the following: A study of interelectrode shorts on cathode walls in the absence of iron addition, is described in Ref. 13. It is found that the number of open gaps on a cathode wall increases linearly or nearly so, with Hall field, Ex, and appears not to be influenced appreciably by anything else, specifically, not by transverse current, $j_{y}$. The voltage across each open gap is found to be about 90 volts $\pm 20 \mathrm{v}$. There follows an evaluation of several mechanisms that might produce such a result, and it is concluded that the mechanism most consistent with the data is one where axial arcs bridge the open gaps and cause the material responsible for the short to vaporize or in some other way be removed.

These findings may have a bearing on the data analyzed under this contract even though most of the data came from runs during which iron was added. Indeed very few runs have been made at the CDIF in recent years without iron.

While iron is being added nearly all gaps appear to be open all of the time. Never-the-less the observations noted above may be pertinent because iron addition may have the effect of simply moving the locus of inter-cathode shorts from the bottom of the slag layer to the top, i.e. from the interface between slag and electrode to the interface between slag and plasma. As noted above, shorting at the top is not necessarily revealed by voltage measured at the bottom since the transverse resistivity of the slag layer is usually relatively high.

Shorting in this case is loosely defined as any axial slag conductivity that could carry a current comparable to what flows into one electrode (i.e. $j y^{\star} p t \star Z$ ) if an electric field small compared to the average axial voltage gradient, $\langle E x\rangle$, were imposed. Attempts to measure axial conductivity in a working generator indicate that it may be as high as $200 \mathrm{mhos} / \mathrm{m}$ while iron is added. This is an average over the thickness of the slag layer, but most of the contribution is presumed to come from the top of the layer. It is observed, for example, that the rate of response to changing feed rate is too rapid to allow for diffusion of iron bearing species from the bottom. In the case of the CDIF, current per electrode is typically about 25 Amps. which, if both electrode walls are doped, results in a voltage gradient;

$$
\begin{aligned}
\mathrm{Ie} /(\sigma * Z * t)=25 /(200 * 2 * & .2 * \\
& =156 \text { volts } / \mathrm{m} \\
& \approx 2.4 \text { volts per electrode gap }
\end{aligned}
$$

These are small compared to the typical <Ex> of about $2000 \mathrm{v} / \mathrm{m}$ and average gap voltage of about $40 \mathrm{v}$, and thus qualify as virtual shorts. Moreover they may be high estimates because only the anode and cathode wall widths, $Z$, are used whereas significant leakage may also occur along other walls as well, in particular that portion of the side walls adjacent to the electrode walls, which must also receive some of the iron added to the flow over the electrodes.

If indeed virtual shorting is occurring during iron addition, then a process similar to that described by pian et al may be occurring and again the 
number of "open" gaps may be proportional to Ex just as it is without iron addition. If this is so then, with or without iron, leakage will appear to be proportional to jy, as will now be shown.

We will start by assuming that with iron injection the number of open gaps will vary with Hall field in the same way as it does without iron. Based upon data collected at the CDIF during run No. 87-SEED-2 (Ref. 14)

No. of open gaps $\approx 10+25 * \operatorname{Ex}(k v)$

Then, if $\mathrm{Ne}$ (tot) is the total number of active electrodes, the number of electrodes in a shorted group, Ns, must average,

$$
\begin{aligned}
N s \approx & N e(t o t) /(1+N o . \text { of open gaps }) \\
& \approx 200 /(11+25 * E x(k v))
\end{aligned}
$$

where $p t$ is the electrode pitch. As a result current will flow across the duct at an angle of about (Ns*pt/Y) where $Y$ is the distance between anode and cathode walls. This results in an apparent axial leakage, Ik, where,

$$
\begin{aligned}
I k & =Y * Z * N s^{*} p t / Y * j y+2 *(Y+Z) *\langle\sigma s>* E x \\
& =N s^{\star} p t * Z^{*} j y+2 *(Y+Z)^{*}\langle\sigma s>* E x \\
& =N s^{*}(I \text { to one electrode })+\text { "Normal" slag leakage. }
\end{aligned}
$$

Inserting Ohm's law for jy and Equation. 1 for Ns,

$$
I k=\frac{200 * p t * Z *(U B+(B-\Phi) * E X)}{\langle 1 / \sigma\rangle^{*}\left(1+B^{2}\right)^{*}\left(11+25^{*} E x\right)}+2 *(Y+Z) *\langle\sigma s\rangle * E x
$$

Thus we obtain an equation with a mix of terms, some dependent on Ex and some on $1 /$ Ex.

Figures 4.1 and 4.2 show plots of this equation. In figure 1 Ik is plotted vs Ex for fixed values of $B, U B,\langle 1 / \sigma\rangle, Y$, and $Z$ typical of the CDIF. It can be seen that the slope of the Ik vs Ex curve, while strongly negative at low Ex, is relatively flat over most of the range of interest.

In Figure $4.2 \mathrm{Ik}$ is plotted vs jy for several values of Ex. For each value of Ex, jy is varied by varying $\langle l / \sigma\rangle$. Here Ik appears to be a function of $j y$, and in most cases curves for different Ex lie nearly on top of one another. Experimental data generally has an amount of scatter comparable to the spread between the curves, excepting the lowest Ex curve, and so would tend to mask the generally weak dependence on Ex.

Note that Figure 4.3 does not seem to exhibit a threshold value of electrode current, $\left(Z^{*} p t * j y\right)$, below which Ik is zero, as the experimental data does. This suggests that the dependence of the number of open gaps on Ex is not quite linear, or that there is a dependence on operating conditions that has not yet been detected.

In any case Figures 4.2 and 4.3 do seem to explain why, experimentally, Ik varies more with jy than Ex. This gives us an insight into the leakage process, suggesting that the effect of iron addition is indeed to shift leakage from the bottom to the top of the slag layer without greatly changing it's magnitude or the mechanism for opening gaps. The shift is important, however, because leakage along the top should not directly be a threat to electrode lifetime. However it 


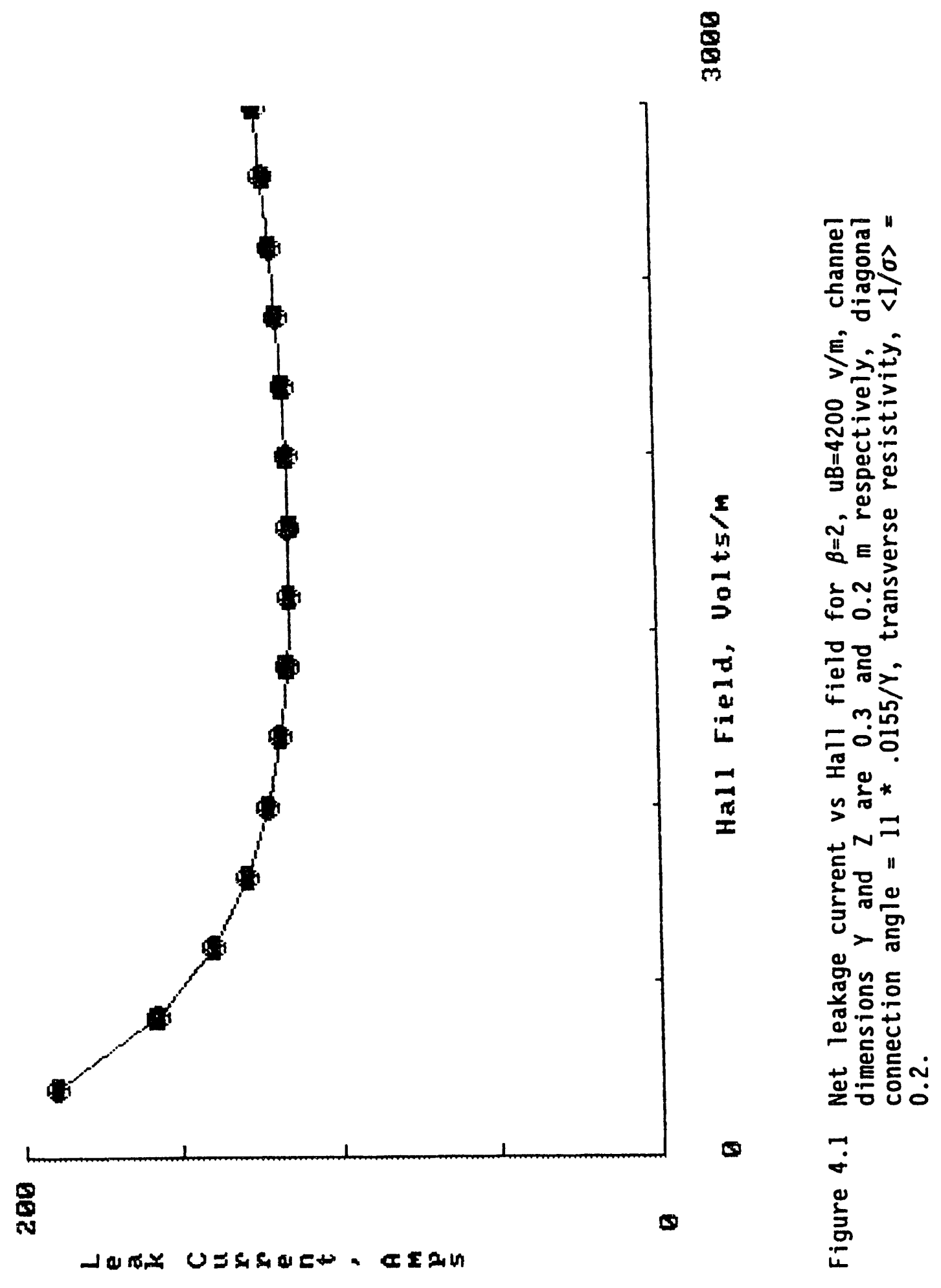




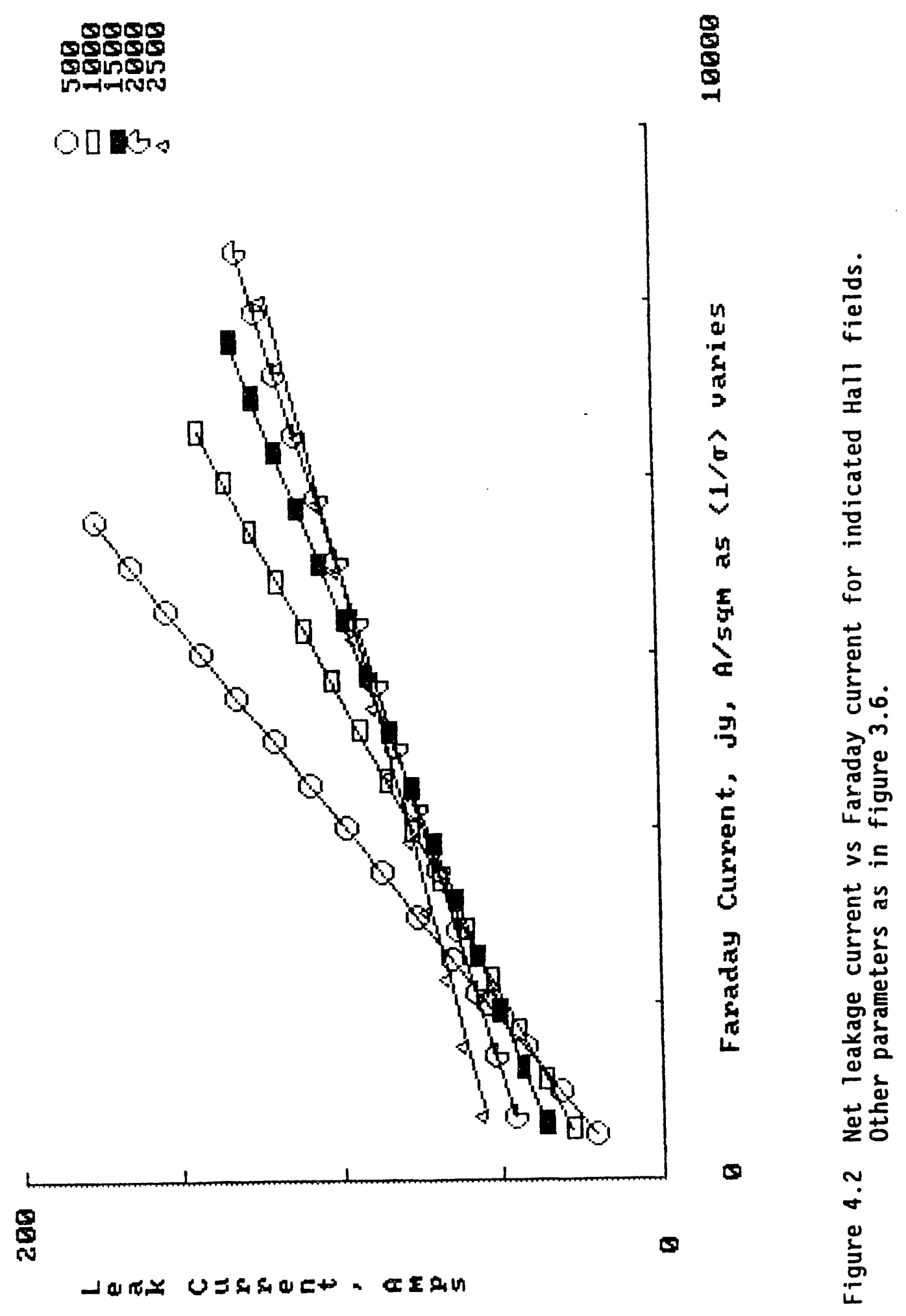


does effect power output. Since leakage seems largest in the front half of the CDIF duct, close to the point where the iron slurry is injected, the leakage could probably be reduced by refinement of the injection system. It is also possible that excessive seed deposition in the front half of the duct is contributing to the effect, which suggests that there may be a need for refinement of the seed injection and mixing process.

A further conclusion is that the leakage should scale as the surface area of the duct and thus have less effect in larger generators, other things being equal.

\subsection{RECOMENDATIONS}

Three methods for indirectly inferring what is happening inside the generator from routinely measured data have been described. These methods have been used because many things that it would be useful to know are not easily measured directly. However uncertainty is inevitable in an indirect process. In order to reduce the uncertainty the following additional data would be valuable.

1) A measurement of conductivity and seed profiles at the burner exit.

2) More reliable static pressure profiles along the channel.

3) A measurement of slag conductivity for flow in the transverse direction, under channel operating conditions.

4) A measurement of the small-signal resistance between a side-wall peg and the plasma.

Some of this data could be obtained with a straightforward refinement of instrumentation already in use, for example the measurement of static pressure. In this case the refinement consists of improving the proceedure for keeping existing pressure taps clear. Redundancy in the form of several taps at each axial location would also be valuable, and greatly help to remove uncertainty as to critical items such as Hall parameter and Mach number distributions.

If high leakage current is a fact and if it is due to high deposition rates of iron slurry and/or seed on the upstream walls of the CDIF channel, then the following are suggested as possible ways to fix the problem.

1) Inject the iron in finer particles evenly distributed over a distance above the cathode wall about equal to the boundary layer thickness at the channel exit. In this way the axial distribution of turbulent mass transport to the wall could be made to be at a rate about proportional to the axial distribution of heat transfer. If this is still too high in front then the initial distribution above the wall might be tailored to correct for it.

A sophisticated injector is obviously required. However, the alternative of inserting injectors at several downstream points takes sophistication too.

2) Determine how and where in the combustion system to inject and vaporize seed so as to ensure that it is uniformly distributed at the entrance to the generator; ir. particular not at any point spun out to the walls. The solution to this well known problem would be greatly facilitated if time and dollars were allocated during burner development to routinely measure conductivity and seed profiles as recomended above under Item 1. 


\subsection{REFERENCES}

1. Pian,C., A. McClain, and N. Schmitt, Avco Quarterly Reports on DOE Contract No. DE-AC22-84PC70507, dated April 1986, pg 93, Jan. 1987, pg 89, April 1987, pg 75.

2. Daniel,V.W. and J. Lohrasbi, Power Loss Mechanisms in Coal-Fired MHD Generators, 27'th SEAM, pp 6.3-1 to 6.3-13, June 27-29, 1989.

3. Nelson, G.L., Y. Lee, I. Stephan, Y.L.C. Wu, J.T. Lineberry, Analysis of CDIF coal Fired Faraday Generator Test Data, 27'th SEAM, June 27-29, 1989,pp4.3-1 to 4.3-16.

4. Rosa, R. J., Leakage Resistance and Current Inferred from CDIF Operating Data, and Addendum, 28'th SEAM, June 1990, Chicago, 111.

5. Rosa, R.J., Leakage Current and Electrode Wear Processes, 29'th SEAM, June 1991.

6. Pollina, R. J., R. Larsen, D. Westpfahl, Topical Report on the Electrical Behavior of Rosebud Coal Slag under MHD Conditions, published by NTIS, 1982. Al so $7^{\prime}$ th International MHD Conference, 1980, and 17' th SEAM, 1978.

7. Ishikawa, M., M. Nakamura, J. Umoto, Interaction Between Electrodynamics and Gasdynamics near Electrode of MHD Generator, 10'th International Conference on MHD, Pp IV.74-80, Tiruchirappalli, India, Dec. 4-8, 1989.

8. Farrar, L. C.,R. J. Rosa, J. C. Tierney, J. D. Teddy, D. J. Trudnowski, Slagging Electrode ArC Data, 26'th SEAM, June 1988.

9. Daniel, V.W., Estimating Plasma/Slag Properties From CDIF Electrical Measurements, AIAA 220nd FluidDynamics, Plasma Dynamics, \& Lasers Conference, June 24-26, 1991 / Honolulu, Hawaii, Paper No. AIAA 91-1515.

10. Daniel, V.W., CDIF Plasma/Slag Calculations, 29'th SEAM, New Orleans, LA, June 1991.

11. Daniel, V.W., et al., Performance Evaluations of MHD Generator Tests at CDIF, 30'th Aerospace Sciences Meeting, Reno, NV, January 1992.

12. Danie1, V.W., Estimating Plasma/Slag Properties in an MHD Channel, AIAA $23^{\prime}$ rd Plasmadynamics \& Lasers Conference, Nashville, TN, July 6-8, 1992.

13. Pian C.C.P.Petty, S.W., McClaine, A. W., Experimental Investigations of Factors Affecting Cathode Wall Resegmentation Frequency, AIAA J. Power and Propulsion, Nov-Dec 91 p 1036.

14. Avco Quarterly Report for January-March 1987, DOE/ET-70507, U.C-90g, p 87) 


\section{ACKNOWLEDGEMENT}

This work is really the work of many people who helped at various levels from preparing the samples, to operating the tests and analyzing the data. A number of these have been co-authors on a large part of this work in earlier incarnations as SEAM presentations ${ }^{1},{ }^{2}$ and as design reviews of the IA4 channel. In particular gratitude is expressed to Stan Petty, the father of the IA4 channel; to Carl Pian, the manager of the AVCO MHD program; to Al Dunton whose perpetual good sense kept us on the straight and narrow; to Buddy Browne11, Jack Fortino, Tony Cuzzupe, and Mike Bartkiewicz in Building 2 whose cheerful, sustained effort made all of this possible; to Larry Farrar whose tenacious direction and 16 hour days made the on-time delivery of the 1 A4 a reality; to Chet Killam and Bob Rose whose patient acceptance of endless redraws and reorders is legendary; to Ned Schmitt, Isaac Sadovnik, and Paul Miedzionoski whose friendship made life richer; to members of the CDIF staff, in particular Gordon Nelson, Wayne Daniels, Jerry Funk, Dave Loftus, and Andrea Hart, for their unfailing cooperation; and to Gary Staats whose permission to allow the material corrosion part of the MSU MHD program to be replaced by work on the AVCO program when one of us (Richard Pollina) moved to AVCO at the start of the three year MSU program. 


\section{APPENDIX A}

As noted, the "simplified" equations used in section 3.2 do not include the effect of leakage transverse to the flow or of resistance in the diagonal connections. For the record, the complete equations used in data analysis are;

$$
B_{s c}=\Gamma d^{\star}\left(j L_{s c} / j y_{s c}-\Phi^{\prime}\right) \star \frac{\left(1-g y^{*} \operatorname{Ees} / u B\right)}{\left(1-\Gamma d^{\star} \operatorname{Ees} / u B\right)}
$$

where;

$$
\begin{aligned}
& \Gamma d=\sigma d /(\sigma d+\sigma y) \\
& g y=1+\sigma y^{*}<1 / \sigma>*\left(1+\beta^{2}\right) \\
& \sigma y=\langle\sigma(\mathrm{slag})\rangle * 2 * t / Z \\
& \sigma d=Y /(Z * p t * R d) \\
& Y \text { and } Z \text { - duct dimensions normal and parallel to } \\
& \text { the magnetic field lines. } \\
& \text { pt }=\text { electrode pitch. } \\
& \text { Rd }=\text { resistance in the diagonal connection. } \\
& \text { Ees }=\text { Ves } / Y \\
& V e s / Y=u_{o c} B-\left(B_{o c}-\Phi^{\prime} * g y\right) * E x_{o c} /\left(j y_{o d} / j y_{s c}-1\right) \\
& \langle 1 / \sigma\rangle_{s c}=\frac{\Gamma d^{*}\left(\left(u_{a c} B-V_{e s} / Y\right) / j y_{a c}-1 / \sigma d\right)}{1+B_{a c}^{2}} \\
& \langle\sigma\rangle_{o c}+\sigma s=j y_{o c} *\left(B_{o c / T d}+\Phi^{\prime}\right) / E x_{o c} \\
& \langle 1 / \sigma\rangle_{o c}=\langle 1 / \sigma\rangle_{\text {oc }} \text { * } \mathrm{rfac} \\
& B_{o c}=B_{s c} * \beta f a c
\end{aligned}
$$

where $r f a c$ and $\beta f a c$ are estimated from CDIF performance calculations. Finally, as before,

$$
\langle\sigma\rangle=r s /\langle 1 / \sigma\rangle
$$

These are the equations actually used in data analysis. However, for reasonable estimates of $\sigma y$ the effect of transverse leakage is generally found to be small. 
DATE FILMED 12128192 
